Smith, David Eugene

Bibliography of the teaching of mathematics, 1900-1912 
UNITED STATES BUREAU OF EDUCATION BULLETIN, 1912, NO. 29

\section{BIBLIOGRAPHY OF THE}

\section{TEACHING OF MATHEMATICS \\ $1900-1912$}

BY DAVID EUGENE SMITH BDA CHARLES COLDZTHER 
 \\ BULLETIN OF THE BUREAU OF EDUCATION.}

(Titles abridged)

1906.

No, 1. The education bill of 1906 for England and Wales. Ana Talman Smith

No. 2. German viows of American education William N. Hailmann.

No. 3. State school systems, Oct, 1, 1904, to Oct. 1, 1906. E O. Elliott.

\section{7.}

No. 1. The continuation school in the United States Arthur J. Jones.

No. 2. Agricultural education James Ralph Jewell.

No. 3. Auxiliary schools of Germany. B Maennel Translated by F. B Dresslar

No. 4. The elimination of pupils from school. Edward L. Thorndike.

1908.

No. 1. On the training of persons to teach agriculture. $L$. $H$. Bailey.

No. 2. List of publications of the United, States Bureau of Education, 1867-1907.

No. 3. Bibliography of education for 1907. J. I. Wyer, jr, and Martha L. Phelps.

No. 4. Music education in the United States. Arthur L. Manchester.

No. 5. Education in Formosa Julean H. Arnold, American consul at $P$ amsui.

No. 6. The apprenticeship system Garroll D. Wright.

No. 7. State school systems, Oct, 1, 1906, to Oct. 1, 1908, E. C. Elliott.

No. 8. Statistics of State universities, etc, $1907-8$

1909.

No. 1. Facilities for study and research in Washington. Arthur T. Hadiey.

No. 2. Admission of Chinese students to Anerican universities John Fryer.

No. 3. Daily meals of school children Caroline $L$. Hunt.

No. 4. The teaching staff of secondary schools. Edward L. Phorodike.

No. 5. Statistics of public, society, and school librarios in 1908 .

No. 6. Instruction in the fine and manual arts. Henry Tumer Bailey.

No. 7. Index to the Reports of the Commissioner of Education, 1867-1907.

No. 8. A teacher's professional library. Classified list of 100 titles.

No. 9. Bibliography of education for $1008-9$.

No. 10. Education for effeciency in railroad ervice. $Y$. Shirlcy Laton.

No. 11. Statistice of State uniyersitiea, ete., 1908-9.

1910.

No. 1. Reform in teaching religion in Saxony. Arley Barthlow Show

No.2. State school systems, Oct, 1, 190s, to Oct. 1, 1909. W. C. Hllott

No. 3. List of publications of the United States Burea of Educalon, 1867-1910.

No. 4. The blological stations of Europe. Charles Atwood Kofoid.

No. 5. American schoolhouses. Jletcher B. Dresslar.

No. 6. Statistics of State universities, etc, $1000-10$

\section{1.}

No. 1. Hibilography of science teaching.

No. 2. Opportunitles for graduate stady in agriculture. A. C. Monahan.

No. 3. Agencles for the improvenent of teachers in service William $\mathrm{C}$. Ruediger.

No. 4. Report of the commission to study the public schools of Baltimore.

5. Ago and grade census of schools and colleges George Drayton Strayer. Graduate work in mathenatics in universities 
UNITED STATES BUREAU OF EDUCATION BULLETIN, 1912, NO. $29 \quad-\quad-\quad \ldots \quad-\quad \ldots \quad$ - WHOLE NUMBER 503

\section{BIBLIOGRAPHY OF THE \\ TEACHING OF MATHEMATICS $1900-1912$}

By DAVID EUGENE SMITH and CHARLES GOLDZIHER

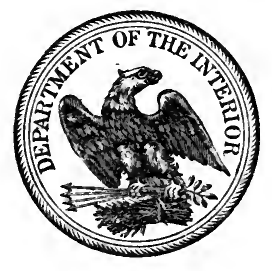





\section{PREFACE.}

Among teachers of mathematics throughout the world there has recently been manifested a notable increase of interest in all educational work. This interest is due in part to the present general agitation in every educational line, in part to the influence of such related departments as physies, in part to the increased demands of industry, and in part to the activities of the International Commission on the Teaching of Mathematics. One result of this interest is a great increase in the literature of the teaching of mathematics, and it seems desirable to present at this time what is at least a beginning toward a bibliography of the subject.

This bibliography has been prepared in part by Professor Charles Goldziher, of Budapest, Hungary, and in part by the writer. Professor Goldziher is a member of various learned societies, a privatdocent at the Polytechnikum at Budapest, the secretary of the Hungarian Commission on the Teaching of Mathematics, and a professor in the State Paedagogium at Budapest. He prepared an extensive bibliography of the recent teaching of mathematics while working at the University of Göttingen, and from this have been made such selections as seemed best to meet the needs of readers, and to it has been added a large number of titles, especially English and American.

The plan has been to include articles on the teaching of mathematics that have appeared since January, 1900, in the various periodicals to which teachers may have access, and to mention works that relate directly to the teaching of mathematics that have been published since that date, but to exclude textbooks. The work will be seen to fall somewhat short of its purpose in that it has been impossible for the authors, in the time at their disposal, to examine all of the literature in the various countries. Nevertheless, it has seemed best, in view of the approaching International Congress of Mathematicians of 1912, to make a beginning of this nature, to the end that others may have a basis upon which to work and that those who wish immediate information upon the literature of the subject may have material at hand to assist them.

The authors desire to recognize the debt they owe to Miss Caroline E. Seely, of New York, in the arduous labor of arranging the material.

David Eugene Suith. 
Digitized by the Internet Archive in 2007 with funding from Microsoft Corporation 


\section{LIST OF ABBREVIATIONS.}

Assoc. Franç. de Grenoble. (Association Française de Grenoble.)

Baseler Verh.andlungen. (Verhandlungen der 49. Versammlung deutscher Philologen und Schulmänner in Basel, 1907. Leipzig, 1905.)

Bay. Zschr. für Realschulw. (Bayrische Zeitschrift für Realschulwesen.)

Blätter für Gymn. Schulw. (Blätter für das Gymnasial Schulwesen.)

Blätter höh. Schulw. (Blätter für das höhere Schulwesen.)

Boll. dell'Assoc. Mathesis. (Bolletino dell'Associazione Mathesis.)

Boll. Mat. (Il Bolletino di Matematica.)

Bull. de la Soc. Franç. de Philosophie. (Bulletin de la Société Française de Philosophie.)

Bull. des Sciences Math. (Bulletin des Sciences mathématiques.)

Bull. des Sci. Math. Elém. (Bulletin des Sciences mathématiques et physiques élémentaires.)

Bull. Soc. Math. (Bulletin de la Société mathématique de France.)

Congr. dei Prof. di Mat. (Congresso dei Professori di Matematica.)

Congr. fra i prof. di mat. delle scuole medie italiane promosso dall' Assoc. Mathesis. (Congresso fra i professori di matematica delle scuole medie promosso dall' Associazione.)

Congr. Int. dei Mat., Roma. (Congresso internazionale dei matematici, Roma.)

Congr. Int. des Math. (Congrès International des Mathématiciens.)

Das Hum. Gymn. (Das Humanistische Gymnasium.)

Encykl. der Math. Wissensch. (Encyclopädie der Mathematischen Wissenschaften.)

Jahresber. D. M. V. (Jahresbericht der Deutschen Mathematiker Vereinigung.)

Klein's Abhandlungen. (Abhandlungen über den mathematischen Unterricht in Deutschland, veranlasst durch die Internationale Mathematische UnterrichtsKommission. Herausgegeben von F. Klein.)

Korrespondenzblatt. (Korrespondenzijatt für die Höheren Schulen Württembergs.)

Középisk. Math. Lapok. (Középiskolai Mathematikai Lapok. Ungarische math. Schülerzeitung.)

Lehrp. und Lehrg. (Lehrproben und Lehrgänge.)

L'Ens. Math. (L'Enseignement Mathématique.)

Magy. Paed. (Magyar Paedagogia.)

Math. Natw. Blätter. (Mathematisch-naturwissenschaftliche Blätter.)

Neue Jahrb. (Neue Jahrbücher.)

Nouv. Annales de Math. (Nouvelles Annales de Mathématiques.)

O. K. T. K. (Országos Középiskolai Tanáregyesületi Közlöny. Berichte der Ungar. Mittelschulprofessorenvereinigung.)

Österr. Mittelschule. (Österreichische Mittelschule.)

Päd. Arch. (Pädagogisches Archiv.)

Päd. Bausteine. (Pädagogische Bausteine.)

Päd. Blätter. (Pädagogische Blätter.)

Päd. Jahrb. (Pädagogisches Jahrbuch.)

Päd. Psych. Studien. (Pädagogische Psychologische Studien.)

Päd. Sammlung. (Pädagogische Sammlung.)

Ped. Tidskr. (Pedagogisk Tidskrift.)

Phys. Zschr. (Physikalische Zeitschrift.) 
Polgárisk. Közl. (Polgáriskolai Közlöny. Berichte für Ungar. Bürgerschulen.)

Preuss. Jahrb. (Preussische Jahrbücher.)

Rev. de l'Ens. des Sciences. (Revue de l'Enseignement des Sciences.)

Schriften der physikal.-ökonom. Gesellschaft zu Königsberg. (Schriften der physikalisch-ökonomischen Gesellschaft zu Königsberg.)

Schweiz. Päd. Zschr. (Schweizerische Pädagogische Zeitschrift.)

Sitzungsber. der Berliner Math. Gesellschaft. (Sitzungsberichte der Berliner mathematischen Gesellschaft.)

Unterrichtsbiätter. (Unterrichtsblätter fur Mathematik und Naturwissenschaften.)

Verhandl. der G. D. N. u. ̈. (Verhandlungen der Gesellschaft Deutscher Naturforscher und Ärzte.)

VerhandI. des III. Int. Math. Congr. in Heidelberg. (Verhandlungen des III. Internationalen Mathematiker Congresses in Heidelberg.)

Weekblad for Gymn. en Midd. Ond. (Weekblad voor Gymnasiaal en Middelbaar Onderwijs.)

Zschr. des Ver. deutscher Ing. (Zeitschrift des Vereines deutscher Ingenieure.)

Zschr. für das gesamte kaufmänn. Unterrichtsw. (Zeitschrift für das gesamte kaufmännische Unterrichtswesen.)

Zschr. für das Gymnasialw. (Zeitschrift für das Gymnasialwesen.)

Zschr. für das Österr. Gymnasialw. (Zeitschrift für das Österreichische Gymnasialwesen.)

Zschr. für das Realschulw. (Zeitschrift für das Realschulwesen.)

Zschr. für Hochschulpäd. (Zeitschrift für Hochschulpädagogik.)

Zschr. für lateinlose höhere Schulen. (Zeitschrift für lateinlose höhere Schulen.)

Zschr. für Math. und Phys. (Zeitschrift für Mathematik und Physik.)

Zschr. für Phil. und Päd. (Zeitschrift für Philosophie und Pädagogik.)

Zschr. für Weibliche Bildung. (Zeitschrift für Weibliche Bildung.)

Zschr. gewerbl. Unt. (Zeitschrift für gewerblichen Unterricht.)

Zschr. Lehrmittelwesen und päd. Literatur. (Zeitschrift für Lehrmittelwesen und pädagogische Literatur.)

Zschr. math. natw. Unt. (Zeitschrift für mathematischen und naturwissenschaftlichen Unterricht.)

Zschr. phys. chem. Unt. (Zeitschrift für physikalischen und chemischen Unterricht.)

(In general, other titles are given in full, save where the abbreviations will readily be understood by English, French, and German readers.) 


\section{BIBLIOGRAPHY OF THE TEACHING OF MATHE- MATICS, 1900-1912.}

\section{GENERAL TOPICS RELATING TO THE TEACHING OF MATHEMATICS.}

1. Adler, A. Über die modernen Methoden im mathematischen Unterrichte. Österr. Mittelschule, 91 (1907).

2. Andrade, J. Quelques observations psychologiques recueillies dans les enseignements scientifiques d'initiation. Congr. dei Mat. Atti del IV. Roma, 1909.

3. Apel, O. Über die Behandlung einiger mathematischen Kapitel im Unterricht. Progr. Städt. Oberrealschule Halle a. S., 1903.

4. Appell, P. L'esprit scientifique et l'enseignement. Rev. de l'Ens. des Sciences, 2, 1908; Revue du Mois, 6 (1908).

5. Archenhold, F. S. Über die Bedeutung des mathematischen Unterrichts in Verbindung mit Reformvorschlägen für den Lehrgang. Congr. Int. dei Mat. Atti del IV. Roma, 1909.

6. Association Suisse de Professeurs de Mathématiques. Compte rendu sommaire de la sixième réunion. Bâle, 20 octobre, 1906.

7. - See the regular department in L'Ens. Math.

8. Az Országos Középiskolai Tanáregyesület mathematikai reformbizottságának jegyzökönyvei. Protokolle der Ungarischen Reformkommission. O. K. T. K., 1906-1909.

9. Baker, A. L., Biklé, C. E., Bruce, G. A., Simons, L. G. and Smith, E. R. General suggestions for marking papers in the state examinations, based upon a passing mark of 60 per cent. Mathematics Teacher, 1 (1908-09): 105.

10. Balás, G. A matematikai tanitás reformjáról. Reform des mathematischen Unterrichts. Vortrag am 15. IV. 1910 in der Lehrerversammlung in Selmecbánya.

11. Beard, W. F. The reform of mathematical teaching. Nature, 62 (1900): 466 .

12. Beck, K. A mathematikai oktatás reformja. Reform des mathematischen Unterrichts. Progr. des Staatl. Obergymn. Erzsébetváros, 1908.

13. Becker, V. Bericht über den II. rheinischen Philologentag zu Bonn, 3-5 Juli, 1908. Neue Jahrb., 11 (1908).

14. Behrendsen. See Schimmack.

15. Beke, M. A matematikai oktatás a szabad tanitásban. Der mathematische Unterricht ausserhalb der Schule. Tagebuch der ungarischen Landeskonferenz für Freien Unterricht in Pécs, 1907. Budapest, 1908.

16. — A matematikai tanitás reformja. Die Reform des mathematischen Unterrichts. Vortrag; O. K. T. K., 40 (1906-07); Beke-Mikola, Sammlung, under Secondary Schools.

17. — Über den jetzigen Stand des mathematischen Unterrichts in Ungarn. Congr. Int. dei Mat. Atti del IV. Roma, 1909.

See also Iskolaorvosok, etc.

18. — and Mikola, S. Abhandlungen über die Reform des mathematischen Unterrichts in Ungarn. Leipzig, 1911. 
19. Berdellé, C. De l'expérience et de l'intuition dans l'enseignement propédeutique de la mathématique. L'Ens. Math., 4 (1902).

20. Bericht der Unterrichtscommission der Gesellschaft Deutscher Naturforscher und Ärzte. Zschr. für lateinlose höhere Schulen, 18 (1907).

21. Bericht über die Wiener Versammlung vom 20. I. 1906. Österr. Mittelschule, 20 (1906).

22. Bettini, B. L'insegnamento della matematica nelle scuole classiche. Congr. dei Prof. di Mat. Atti del II. Livorno, 1902.

23. Biklé, C. E. See Baker, A. L.

24. Blakslee, T. M. Culture course in mathematics. School Science and Mathematics, 5 (1905): 645, 752; 6 (1906): 58, 133.

25. Bobynin, V. A propos de l'enquête sur la méthode de travail des mathématiciens; réflexions sur les réponses aux questions 4 et 5 . L'Ens. Math., 9 (1907): 135; aux questions 11, 12, et 13. L'Ens. Math., 9 (1907) 389.

26. — Cas particuliers d'emploi dissimulé de la méthode expérimentale dans les temps les plus récents. L'Ens. Math., 9 (1907).

27. L L'enseignement mathématique en Russie. L'Ens. Math., 5 (1903); 6 (1904).

28. Boehm, E. Stellen die Vorschläge der Unterrichtskommission der Gesellschaft Deutscher Naturforscher und Ärzte einen Fortschritt auf dem Wege der Schulreform dar? Neue Jahrb., 18 (1906).

29. Bonnesen, T. La réforme de l'enseignement des mathématiques élémentaires. Rivista di Scienza, 2 (1907).

30. Breckenridge, w. E. Checks, their use and abuse. Mathematics Teacher, 1 (1908): 17 .

31. British Association Committee on the Teaching of Mathematics. Report. Mathematical Gazette, 2: 197.

32. Brocke, E. Die Frage der Neugestaltung des mathematischen Unterrichts und die Strassburger Vorschläge von 1895. Zschr. math. natw. Unt., 38 (1907): 375 .

33. - Die neue Schulmathematik in methodischer Hinsicht. Vortrag in Basel. s. das Grossmann'sche Referat in Zschr. math. natw. Unt., 39 (1908).

34. Broomell, B. L. International commission on the teaching of mathematics. Mathematics Teacher, 1 (1908): 14.

35. Buchrucker, B. Über Schulwerk und Schulbetrieb der Mathematik. Neue Jahrb., 10 (1907).

36. Buhlmann, B. Über die Reformbestrebungen auf dem Gebiete des mathematischen Unterrichts. Päd. Arch., 47 (1905).

37. Burstall, S. A. The place of mathematics in the education of girls and women. Reports of the British Subcommission, 18.

38. Buyse, O. Méthodes américaines d'éducation générale et technique. Paris, 1908:

39. Campbell, J. The teaching of mathematics. American Federation of Teachers of the Mathematical and Natural Sciences, 1909. Bulletin, no. 2.

40. Cardinaal, J. L'enseignement mathématique en Holland. L'Ens. Math., $2(1900): 317$.

41. - Rapport sur l'enseignement mathématique dans les Pays Bas. Rapport de la sous-commission hollandaise, Delft, 1911.

42. Ciamberlini, C. Contributo ad un miglioramento didatica dei libri di testo di matematica elementare. Boll. Mat., 3 (1904).

43. Coar, H. I. Routine work in mathematics. School Review, 8 (1900): 271.

44. Cobb, H. E. Personal observations of the teaching of mathematics in Prussia. School Science and Mathematics, 7 (1907): 518. 
45. Cobb, H. E. The real problem of the teacher of mathematics. National Education Association of the United States. Journal of proceedings and adresses, 1904. p. 518.

46. Collins, J. V. Homemade or inexpensive mathematical apparatus. School Science and Mathematics, 7 (1907): 524.

47. - Mathematics and the doctrine of formal discipline. School Review, 14 (1906): 604 .

48. —_ Sur la méthode d'enseignement en Amérique. L'Ens. Math., 8 (1906): 146.

49. Commission Internationale de l'Enseignement des Mathématiques. I Les travaux préparatoires. Genève, Kundig, 1910. Circulaire no. 4. Etat des travaux au $1^{\text {er }}$ mars 1911. Par le sécrétaire-général, H. Fehr. L'Ens. Math., 13 (1911): 122. Séance de clôture à Motterone. L'Ens. Math., 13 (1911): 509. Annexe: Rapport de M. Young. L'Ens. Math., 13 (1911): 471.

See also Fehr., H., and the reports of the various countries.

50. Committee on the Unification of Mathematics. Report. Discussion. School Science and Mathematics, 10, 1910.

51. Comstock, C. E. A few deficiencies in the teaching of mathematics. School Science and Mathematics, 7 (1907): 12, 97.

52. Condit, I. S. Mathematics as a factor in character building. School Science and Mathematics, 7 (1907): 640.

53. Congress of Mathematicians. See 56 .

The proceedings of the Cambridge Congress of 1912 are in process of publication.

54. Congresso della "Mathesis," Società Italiana di Matematica. Atti del $11^{\circ}$. Firenze, 16-23. X. 1908. Padova, 1908; Lietzmann's Rubrik in Zschr. math. natw. Unt., 40 (1909).

55. Congresso dei Professori di Matematica. Atti del II. Livorno, 1902.

55. Congresso Internationale dei Matematici. Atti del IV. Roma, 1908. Reports by Klein-Gutzmer-Lehrerausbildung; Gutzmer-Germany; BorelFrance; Godfrey-England; Smith-United States; Suppantschitsch-Austria; Beke-Hungary; Fehr-Switzerland; Stéphanos-Greece; ContiItaly; Galdéano-Spain.

57. Conti, A. See Enriques.

58. Coolidge, J. I. The opportunities for mathematical study in Italy. Bulletin of the American Math. Society, New York, 1904.

59. Csada, I. A mathematikai oktatás reformja. Zur Reform des mathematischen Unterrichts. Nevelés, 26 (1909).

60. Cubberly, E. P. See Influences tending to improve the teaching, etc.

61. Czuber, E. Die Frage der Reform des mathematischen Unterrichts auf der Meraner Naturforscherversammlung. Österr. Mittelschule, 20 (1907).

62. Daninger, J. Mathematik und die Phantasie. Österr. Mittelschule, 22 (1908).

63. Dankwortt, A. 18. Hauptversammlung des Vereins zur Förderung des mathematisch-naturwissenschaftlichen Unterrichts in Freiburg i. B. Zschr. math. natw. Unt., 40 (1909): 417, 542, 571.

64. Dausat, - Eléments de méthodologie mathématique. 2e. ed. Paris, 1908.

65. Démonstrations et explications dans l'enseignement élémentaire. L'Ens. Math., 9 (1907).

66. Discussion of reform in the teaching of mathematics. Mathematical Gazette, 2 (1902).

67. Discussion on proposed improvements in the teaching of elementary mathematics. Mathematical Society, Edinburgh. Proceedings, 1902. v. 20. 
68. Dittrich, E. Die Ursachen der Genauigkeit in der Mathematik. Progr. Wittingau (Bohemian), (1908-09).

69. Diusing, K. Zur Methodik des mathematischen Unterrichts. Vortrag. Zsschr. gewerbl. Unt., 23 (1908).

70. Eingabe vom. 6. XII. 1907. der fünf Vereine: Deutsche Ingenieure, Realschulmänner, Lateinloses höheres Schulwesen, Schulreform, Deutsche Chemiker an den preussischen Unterrichtsminister Dr. Holle.

71. Enquête sur la méthode de travail des mathématiciens. L'Ens. Math., 7 (1905): 387,$473 ; 8$ (1906): 43, 217, 293, 383, 463; 9 (1907): 123, 128, 204, 306, 473; 10 (1908): 152, 171; also Paris, 1908.

72. Enriques, F., Severi, F. and Conti, A. Estenzione e limiti dell'insegnamento della matematica. Boll. Mat., 2 (1903).

73. Erdös, L. A mathematikai tanitás reformjáról. Über die Reform des mathematischen Unterrichts. Progr. der Oberrealschule in Zsolna, (1906-07).

74. Examinations in mathematics other than those set by the teacher for his own classes. International Commission on the Teaching of Mathematics. American report. Committee no. VII. T. S. Fiske, chairman. Washington, D. C., 1911. 72 p. $8^{\circ}$. (U.S. Bureau of Education. Bulletin, 1911, no. 8.)

75. Une exposition mathématique au Musée Pédagogique de l'Université de Columbia. L'Ens. Math., 11 (1910).

76. Fack, Mr. Zur didaktischen Darstellung von Stoffen aus der niederen und höheren Mathematik. Progr. Weimar, 1907; und Beiträge zur Lehrerbildung und Lehrerfortbildung, Gotha, 37 (1907).

77. Faragó, A. A mathematika jelentösége az oktatásban. Die Bedeutung der Mathematik im Unterricht. Progr. der Staatl. Oberrealschule, Sopron, 1908.

78. —_ Reformmozgalmak a mathematikai oktatás terén. Reformbewegungen im Gebiete des mathematischen Unterrichts. Vortrag in der Soproner Pädagogischen Gesellschaft, 1906.

79. Fédération Américaine des Professeurs de Sciences Mathématiques et Naturelles. L'Ens. Math., 11 (1908): 176.

80. Fehr, H. A perçu général. Rapports de la sous-commission suisse, 2.

81. — Compte rendu des séances de la Commission Internationale faites à Bruxelles du 10 au 16 août 1910. L'Ens. Math., 12 (1910); also published separately as Circulaire No. 3.

82. _- Compte rendu du Congrès de Milan, 18-21 septembre, 1911. Geneva, 1911; L'Ens. Math., 13 (1911).

83. — L'enquête de "L'Enseignement Mathématique" sur la méthode de travail des mathématiciens. Verhandl. des III. Int. Math. Congr. in IIeillelberg, Leipzig, 1905.

84. - Le $4^{\mathrm{e}}$ Congrès International des Mathématiciens, Rome, 1908. L'Ens. Math., 10 (1908).

85. — Die Vorarbeiten. Les travaux préparatoires. (In French and German.) Rapports de la sous-commission suisse, 1.

See also Commission Internationale.

86. Festschrift zur 48. Versammlung deutscher Philologen und Schulmänner in Basel, 1907.

87. Field, F. Elementary mathematics. School Review, 14 (1906): 350.

88. Fiske, T. S. See Examinations in mathematics, etc.

89. Fontené, G. Rapports sur l'enseignement des mathématiques. Rev. de L'Ens. des Sciences, 2 (1908); 4 (1910); 5 (1911).

90. Forth, C. J. The teaching of mathematics. Nature, 65 (1901-02): 272.

91. French, J. S. What study of mathematics is needed by the man of average education for practical life? National Education Association of the United States. Journal of proceedings and addresses, 1904. p. 510. 
92. Fricke, R. Über Reorganisationsbestrebungen des mathematischen Elementarunterrichts in England. Jahresber. D. M. V., 13 (1904).

93. Fröhlich, K. See Iskolaorvosok, etc.

94. Galdéano, Z. G. Algunas consideraciones sobre filosofia y enseñanza de la matémática. Zaragoza, 1907.

95. — Estudios de critica y pedagogia matémáticas. Zaragoza, 1900.

96. — Exposición sumaria de la teoriás matémáticas. Zaragosa, 1907

97. - Nuova contribuzione à la enseñanza matémática. Zaragoza, 1910.

98. — Quelques mots sur l'enseignement mathématique en Espagne. Congr. Int. dei Mat. Atti del IV. Roma, 1909.

99. _ Subcommission española parie el progreso de la enseñanza matémática. Discurso inaugurale, Zaragoza, 1909.

100. Geissler, K. Beiträge zur Vertiefung und Verbindung des exakten Unterrichts durch Unendlichkeit und Continuität. Baseler Verhandlungen.

101. _ Das Streben nach Zusammenhang im mathematischen Unterricht und seine erziehliche Bedeutung. Neue Jahrb., 7 (1904).

102. - Wann verliert die Mathematik als Unterrichtsgegenstand ihren eigentlichen und hervorragenden Wert? Neue Jahrb., 8 (1905).

103. Gérard, I. Sur les démonstrations mathématiques. Bull. des Sciences ilath. Elém., 12 (1907).

104. Gesamtbericht und einzelne Berichte der Unterrichtskommission der Gesellschaft Deutscher Naturforscher und Ärzte. See Gutzmer.

105. Gheury, M. E. J. An arbitrary veto. Mathematical Gazette, 5 (1909).

106. Gibson, G. A. The methods of mathematics. Nature, 82 (1909-10): 109.

107. Godfrey, C. The teaching of mathematics. A compromise. Mathematical Gazette, 2 (1901).

108. — The teaching of mathematics in English public schools for boys. Congr. Int. dei Mat. Atti del IV. Roma, 1909; Mathematical Gazette, 4 (1908); L'Ens. Math., 10 (1908): 459.

See also Langley, E. M.

109. Goldziher, K. Általános bevezetés a mathematika tanitásának módszertanába. Allgemeine Einleitung in die Methodik des mathematischen Unterrichts. Polgárisk. Közl., 14 (1910).

110. — Jelentés a külföldi reformtörekvésekröl. Bericht über die ausländischen Reformbestrebungen. See Beke-Mikola, Sammlung, under Secondary Schools.

111. — Klein, Felix, paedagogiai törekvései. Die pädagogischen Bestrebungen Felix Klein's. O. K. T. K., 39 (1905-06).

112. — A mathematikai laboratoriumról. Über das mathematische Laboratorium. O. K. T. K., 41 (1908); in deutscher Übersetzung, Unterrichtsblätter, 14 (1908); English translation by H. E. Cobb, School Science and Mathematics, 8 (1908): 753 .

113. — A mathematika tanitásának fokozatai. Die Entwickelungsstufen des mathematischen Unterrichts. Magy. Paed., 19 (1910); Päd. Arch., 52 (1910).

114. — Reformtörekvések a mathematikai oktatás terén. Reformbestrebungen im Gebiete des mathematischen Unterrichts. Magy. Paed., 18 (1908-09); also Budapest, 1908.

115. — Ungarische mathematische Schülerzeitung. Lietzmann's Rubrik in Zschr. math. natw. Unt., 41 (1910).

116. — Zeitgemässe Umgestaltung des mathematischen Unterrichts. Päd. Zeit., 1905.

See also Iskolaorvosok, etc. 
117. Göransson, E. Nyare riktlinger för mathematikundervisningen. Neuere Gesichtspunkte für den mathematischen Unterricht. Stockholm, 1908-09.

118. — - Svar på genmäle. Ped. Tidskr., 45 (1909).

119. — and Meyer, A. Framåt eller tillbaka. Ped. Tidskr., 45 (1909).

120. Götting, E. See Holzmüller, C.

121. Grave, W. Beiträge zur rationellen Didaktik der mathematischen Disciplinen. Dissertation. Leipzig, 1908.

122. Grévy, A. Examens et examinateurs. Rev. de l'Ens. des Sciences, 1 (1907).

123. Grossmann, M. Bericht über eine in Basel veranstaltete Besprechung der Reformvorschläge der Unterrichtskommission der Gesellschaft Deutscher Naturforscher und Ärzte. Zschr. math. natw. Unt., 39 (1908): 79.

124. Guadagno, P. Sul metodo d'insegnamento per la matematica. . 1903.

125. Guldberg, A. L'enseignement des mathématiques en Norvège. L'Ens. Math:, 7 (1905): 433.

126. Günther, S. Le développement historique de l'enseignement mathématique en Allemagne. L'Ens. Math., 2 (1900): 237.

127. Gutzmer, A. Bericht über die Tätigkeit des deutschen Ausschusses im Jahre 1908. Schriften des Deutschen Ausschusses, Leipzig, 1909; Zschr. math. natw. Unt., 40 (1909): 359.

128. _ _ Bericht über die Tätigkeit des Deutschen Ausschusses im Jahre 1909. Schriften des Deutschen Ausschusses, Leipzig, 1910.

129. — Die Tätigkeit der Unterrichtskommission der Gesellschaft Deutscher Naturforscher und Ärzte. Gesamtbericht, enthaltend die Vorverhandlungen auf den Versammlungen in Cassel und Breslau, sowie die seitens der Kommission den Versammlungen in Meran, Stuttgart und Dresden unterbreiteten Reformvorschläge. Leipzig, 1908.

130. — Über die Reformbestrebungen auf dem Gebiete des mathematischen Unterrichts in Deutschland. Congr. Int. dei Mat. Atti del IV. Roma, 1909.

131. Hadamard, J. Réflexions sur la méthode heuristique. Revue Générale des Sciences, 16 (1905).

132. Hawkes, H. E. Educational values in mathematical teaching. Mathematics Teacher, 4 (1911-12): 75.

133. Hawkins, C. Examinations from the school point of view. Reports of the British Subcommission, 10.

134. Heaviside, O. The teaching of mathematics. Nature, 62 (1900): 548.

135. Heckscher, I. Reformforslag for undervisningen i mathematik i Preussen. Nyt Tidsskrift, 17 (1906).

136. Hobson, E. W. The democratisation of mathematical education. Nature, 88 (1911-12): 396.

137. — Tendencies of modern mathematics. Educational Review, 40 (1910): 524.

138. Hoefinghoff, E. Über die neueren Bestrebungen zur Umgestaltung des mathematischen Unterrichts. Progr. Gymn. zu Wittstock, 1909.

139. Höfler, A. Didaktik des mathematischen Unterrichts. Leipzig, 1910.

140. — Die Meraner Forderungen der deutschen Naturforscher und die neuesten österreichischen Lehrpläne vom Jahre 1908-1909. Päd. Arch., 52 (1910).

141. _ Die Reformbewegungen des realistischen Unterrichts in Deutschland und Österreich. Drei Vorträge zur Mittelschulreform, Wien, 1908.

142. Holmes, H. I. Elementary mathematics in evening schools. Mathematical Gazette, 5 (1909-10): 200.

143. Holzmüller, G. and Götting, E. Discussion. Jahresber. D. M. V., 14.

144. Hovestadt, H. See Killing, W. 
145. Influences tending to improve the work of the teacher of mathematics in the United States. International Commission on the Teaching of Mathematics. American report. Committee no. VIII. E. P. Cubberley, chairman. Washington, D. C., 1912. (U. S. Bureau of Education. Bulletin, 1912, no. 13.)

146. Iskolaorvosok és egészségtantanárok szakbizottsága: 1907 nov. 8 ülés jegyzökönyve. Protokoll der Sitzung vom 8 Nov. 1907 der Fachkommission von Schulärzten und Hygieneprofessoren; Vortrag von Beke und Bemerkungen von Rades, Szabó, Mikola, Goldziher, und Fröhlichk. Egészség, 22 (1908.)

147. Jackman, W. S. The correlation of mathematics. Educational Review, 25 (1903): 249.

148. Jackson, C. S. and Ward, F. L. Reform in mathematical teaching. Nature, 65 (1901-02): 558 .

149. Jelentés a bizottság határozatairól és javaslatairól. Bericht ‘über die Beschlüsse und Vorschläge der Reformkommission; see Beke-Mikola, Sammlung, under Secondary Schools.

150. Jones, F. T. Some experiences in laboratory mathematics and their results. School Science and Mathematics, 5 (1905): 406.

151. Jourdain, P.E.B. The study of mathematics. Mathematical Gazette, 4 (1908).

152. Joxe, A. L'éducation des facultés d'observation. Rev. de l'Ens. des Sciences, 3 (1908).

153. Juckel, Gy. Mathematikai oktatásunk reformjáról. Reform des mathematischen Unterrichts. Kereskedelmi Szakoktatás, 18 (1909-10).

154. Junge, G. Der neue österreichische Lehrplan für Mathematik. Päd. Arch., $52(1910)$.

155. — Die unpraktische Schulmathematik. Päd. Arch., 49 (1907).

156. Kármán, T. A német természettudósok ez évi dresdeni nagygyüléséröl. Über die Dresdener Tagung der Gesellschaft Deutscher Naturforscher und Ärzte. Magyar Mérnök-és Épitész-Egylet Heti Értesitöje, 30 (1907).

157. Karpinski, L. C. Reform in the teaching of mathematics. School Review, 17 (1909): 267.

158. Keyser, C. J. Exercises in thinking about number and space. Educational Review, 26 (1903): 246, 394, 486; 27 (1904): 36, 160, 270.

159. Killing, W. and Hovestadt, H. Handbuch des mathematischen Unterrichts. Leipzig, 1911.

160. Klein, F. Elementarmathematik vom höheren Standpunkte aus. I, II. Leipzig, 1908, 1909.

161. Kleinpeter, H. Zur Reformfrage des mathematischen Unterrichtes. Zschr. für das Realschulw., 31 (1906): 261.

162. Klug, J. Zum mathematischen Unterricht. Blätter für das Gymn. Schulw., 41 (1905): 722.

163. Knabe, K. Die Meraner Vorschläge in practischer Beleuchtung. Zschr. math. natw. Unt., 38 (1907).

164. Koch, E. H. Training for efficiency in elementary mathematics. Mathematics Teacher, 3 (1910-11): 170.

165. Kollros, L. La mathématique pure et l'approximation. L'Ens. Math., 8 (1906).

166. Kühnemann, F. Das geschichtliche Moment im mathematischen Unterricht. Schriften der physikal-ökonom.. Gesellschaft, Königsberg, 47 (1906).

167. Laisant, C. A. L'éducation fondée sur la science. Paris, 1905.

168. - Initiation mathématique. Paris, 1906; Italian transl. by G. Lazzeri, Firenze, 1908.

169. — La mathématique; philosophie, enseignement. Paris, 1907.

170. — Le rôle sociale de la science. L'Ens. Math., 6 (1904). 
171. Langley, E. M., Godfrey, C. and Siddons, A. W. The teaching of matheInatics. Mathematical Gazette, 2 (1901-1904): 105.

172. Lanner, A. Beiträge zur Didaktik des mathematischen Unterrichts in den Gymnasien. Zschr. math. natw. Unt., 43 (1912).

173. Lechnitczky, Gy. A mathematika-tanitás reformja. Die Reform des mathematischen Unterrichts. Magyar Tanitóképzö, 22 (1907); 23 (1908).

174. Lehmer, D. N. On the use and abuse of textbooks. School Science and Mathematics, 8 (1908): 13.

175. Leitsätze aufgestellt auf der westphälischen Direktorenkonferenz von 12 . bis 14 . Juni 1907. Unterrichtsblätter, 13 (1907).

176. Lengauer, J. Über neuere Vorschläge zur Reform des mathematischen Unterrichtes. Blätter für das Gymn. Schulw., 41 (1905): 646.

177. Lessing, Th. Paedagogie und Psychologie der Mathematik. Zschr. für experimentelle Pädagogik, 9 (1909).

178. Levi, B. Esperienza e intuizione in rapporta alla propedeutica matematica. Boll. Mat., 6 (1907).

179. Lexis, W. Das Unterrichtswesen im Deutschen Reich, 1-4. Berlin, 1904.

180. Leygues, —. Lettre au Président de la Commission de l'enseignement de la Chambre des Députés. In Hachette's edition of the programmes of instruction for 1902.

181. Lietzmann, W. $80^{\text {te }}$ Versammlung deutscher Naturforscher und Ärzte in Cöln. Math. Natw. Blätter, 5 (1908).

182. - Berichte und Mitteilungen, veranlasst durch die Internationale Unterrichtskommission, I-VI. Leipzig and Berlin, 1909-1911.

183. —_ Bericht über die Tätigkeit des Deutschen Ausschusses im Jahre 1910. Schriften des Deutschen Ausschusses, Leipzig, 1911; Zschr. math. natw. Unt., 42 (1911).

184. _ Le mouvement réformateur de l'enseignement mathématique en Allemagne. Rev. de l'Ens. des Sciences, (1908).

185. _ Sammelbericht über neuere Veröffentlichungen auf dem Gebiete des mathematischen Unterrichts. Päd. Arch., 5 (1909).

186. — Stuttgarter Vorschläge der Unterrichtscommission. Math. Natw. Blätter, 4 (1907).

187. Lindemann, F. Lehren und Lernen in der Mathematik. Rectoratsrede, München, 1904.

188. Lodge, O. Russische Übersetzung der Vorlesungen über "Schulunterricht und Schulreform." Mosk. Päd. Sammlung, 1907.

189. Löffler, E. Zur Reform des mathematischen Unterrichts. Korrespondenzblatt, 19 (1912).

190. Loos, J. Enzyklopädisches Handbuch der Erziehungskunde, herausgegeben unter Mitwirkung von Gelehrten und Schulmännern. 2 Bände. Wien, 1906.

191. Lorey, W. Die mathematischen Wissenschaften und die Frauen. Vortrag. Frauenbildung, 8 (1908),

192. Loria, G. Sulle riforme scholastiche da compiersi e in particolare su quelle relative all'insegnamento della matematica. Lettera al Prof. Conti. Boll. Mat., 5 (1906).

193. — Sur l'enseignement des mathématiques élémentaires en Italie. L'Ens. Math., 7 (1905): 11.

194. — Vergangene und künftige Lehrpläne. Leipzig, 1906. The Italian original: Programmi del passati e programmi per l'avvenire in Boll. dell' Assoc. Mathesis, 1905.

See also Réformes à accomplir, etc., Kisposte, ete. 
195. McCormack, T. J. Why do we study mathematics: a philosophical and historical retrospect. National Education Association of the United States. Journal of Proceedings and Addresses, 1910. p. 505.

196. Mach, E. Über den relativen Bildungswert der philosophischen und mathematisch-naturwissenschaftlichen Unterrichtsfächer. Wien, 1886; Neue Auflage, 1908.

197. Mahistre, G. L'enseignement mathématique. 1901.

198. Mannoury, G. Methodisches und Philosophisches zur Elementar-Mathematik. Haarlem, 1909.

199. Marotte, F. L'évolution actuelle de l'enseignement mathématique en Angleterre et en Allemagne. Bull. des Sciences Math., 39 (1905).

See also Réformes à accomplir, etc.

200. Mathematical Association. Annual meeting, London, 1902. Discussion on the reform in the teaching of mathematics. Mathematical Gazette, 2(1901-4): 129 .

201. _ Reports of the committee, 1902-8. London, 1909.

The reports appeared separately in Mathematical Gazette.

202. Mathematics in Austria. Nature, 84 (1910): 394.

203. Mathematics in English schools. Nature, 88 (1911-12): 226.

204. Mayer, _ _ Bericht über die Jahresversammlung des "Vereins realistischer Lehrer" Württembergs. Zschr. für lateinlose höhere Schulen, 19 (1908).

205. Méray, C. L'enseignement des mathématiques. L'Ens. Math., 3 (1901): 172.

206. Metzler, W. H. Formal discipline. Mathematics Teacher, 3 (1910-11): 9.

207. — Mathematics for training and culture. Mathematics Teacher, 2 (1909-10): 47.

208. Where shall we place the emphasis? Mathematics Teacher, 1 (1908-09): 2.

209: Mevius, w. Rechnen und Raumlehre. Band II. Heft 1 von Gehrig, H., Methodik des Volks- und Mittelschulunterrichts. Leipzig, 1906.

210. Meyer, A. See Göransson, E.

211. Meyer, U. Neuerscheinungen zur Reform des mathematischen Unterrichts. Frauenbildung, 10 (1911).

212. Mikola, S. A középiskolai mathematikai oktatás reformja ügyében keletkezett bizottság megalakulásának és müködésének története. Entwickelungsgang und Thätigkeit der ungarischen Reformkommission. See Beke-Mikola, Sammlung, under Secondary schools.

213. — A mathematikai tanítás reformja. Reform des mathemacischen Unterrichts. Vortrag. O. K. T. K., 43 (1909).

214. — Reformmozgalmak a mathematikai tanítás terén Angliában. Reformbewegungen im Gebiete des mathematischen Unterrichts in England. O. K. T. K., 37 (1903).

S'ee also Beke, and Iskolaorvosok, etc.

215. Miller, G. A. On some of the symbols of elementary mathematics. School Science and Mathematics, 7 (1907): 406.

216. - Recent tendencies in mathematical instruction. Popular Science Monthly, 68 (1906).

217. — Reform in mathematical instruction. Science, 24 (1906).

218. - Some questionable terms and definitions in elementary mathematics. School Science and Mathematics, 8 (1908): 571.

219. Misar, W. Versuch einer Zusammenstellung der von Schüler in der Mathematik am häufigsten begangenen Fehler. Zschr. für das Realschulw., 28 (1903): 267. 
220. Mootschek, W. and Philippowitsch, F. Pedagogy of mathematics (Russian). St. Petersburg, 1910.

221. Muir, D. The reform of mathematical teaching. Nature, 62 (1900): 389.

222. Müller, H. Rechnen und Mathematik. Handbuch für Lehrer höherer Schulen. Leipzig, 1906.

223. Munchoff, E. R. von. Mathematics for service. Mathematics Teacher, 2 (1909-10): 116.

224. Myller, A. Incercări de reformă a invătămintului matematic în Germania. Rivista de Filosofie și Pedagogie, Bucuresti, 1 (1906).

225. Nath, M. Die Reformvorschläge der Unterrichtscommission der deutschen Naturforscherversammlung. Preuss. Jahrb., 1907.

226. Natucci, A. Risposte ad alcune domande del questionario per la riforma da compiersi nell'insegnamento della matematica. Boll. Mat., 5 (1906).

227. Neuhoff, $H$. Der reele Wert der Mathematik, insbesondere an Gymnasien. 13lätter höh. Schulw., 24 (1907).

228. Niewenglovski, B. Rapport sur l'aggrégation des sciences mathématiques. Rev. de l'Ens. des Sciences, 1909, and Revue Universitaire, 1 (1909).

229. Oberle, $\mathrm{K}$. A mathematikai oktatás reformjáról. Über die Reform des mathematischen Unterrichts. Magyar Tanítóképzö, 23 (1908).

230. Osgood, W. F. See Report of the American Commissioners.

231. Ottermann, C. The teaching of mathematics. Science, 28 (1908).

232. Packard, J. C. A proposed reform in mathematics. Educational Review, 39 (1910): 455 .

233. Palatini, F. Estensioni e limiti dell'insegnamento della matematica. Congr. dei Prof. di Mat. Atti del III. Torino, 1903.

234. Paulsen, F. Nochmals die Reformfrage der Unterrichts-Kommission der Gesellschaft Deutscher Naturforscher und Ärzte. Monatschrift für höhere Schulen, 6 (1907).

235. Penseler, G. Eine Ausstellung von Schülerarbeiten aus der Mathematik, etc. Monatschrift für höhere Schulen, 10 (1911).

236. Perry, J. A course of elementary mathematics. School World, 1900.

237. _ Discussion on the teaching of mathematics. British association meeting at Glasgow, 1901; edited by Perry. London, 1902.

238. — England's neglect of science. London, 1900.

239. - The rational teaching of mathematics. Nature, 1901.

240. - The teaching of mathematics. Nature, 62 (1900): 317; 64 (1901): 592; 65 (1901-02): 484; School World, 3 (1901); Educational Review, 23 (1902): 158.

241. Petrini, H. Matematiken i skolan. Ped. Tidskr., 41 (1905).

242. Pfitzner, P. Über den didaktischen Wert zusammenhängender Aufgabengruppen im mathematischen Unterricht. Progr. Gymn. z. h. Kreutz, Dresden, 1908.

243. Philippowitsch, F. See Mootschek, W.

244. Pierpont, J. Mathematical instruction in France. Bulletin of the American Mathematical Society, 6 (1900): 225.

245. Pietzker, F. Bericht über den Stand der Arbeiten der von der Breslauer Naturforscherversammlung eingesetzten Unterrichtskommission. Unterrichtsblätter, 11 (1905).

246. —_ L'enseignement mathématique en Allemagne pendant le XIX $\mathrm{X}^{\mathrm{e}}$ siècle. L'Ens. Math., 3 (1901): 2, 77.

247. — L'insegnamento della matematica nelle scuole medie della Germania. Boll. Mat., 1 (1902). 
248. Pietzker, F. Die Stellung der Fachkreise zu den Vorschlägen der von der Naturforschergesellschaft eingesetzten Unterrichtskommission. Vortrag. Unterrichtsblätter, 12 (1906).

249. — Über den wissenschaftlichen Charakter des elementarmathematischen Unterrichts. Unterrichtsblätter, 16 (1910).

250. — Die Vorschläge der Unterrichtskommission der Gesellschaft Deutscher Naturforscher und Ärzte für die Reform des mathematischen Unterrichts. Südwestdeutsche Schulblätter, 24 (1907).

251. Pincherle, S. See Risposte, etc.

252. Plasche, R. Beiträge zur Methodik des mathematischen Unterrichts. Progr. Staatsgymn. Kgl. Weinberge, 1911.

253. Pochin, E. A. N. Experimental mathematics. Philosophical Magazine, Series VI, 14 (1907).

254. Poincaré, H. Science et méthode. Paris, 1908.

255. — La valeur de la science. 2e éd., Paris, 1909.

256. Poske, F. Noch ein Wort über die Reformvorschläge der Unterrichtskommission der Gesellschaft Deutscher Naturforscher und Ärzte. Monatschrift für höhere Schulen, 6 (1907).

257. Pringsheim, A. Über Wert und angeblichen Unwert der Mathematik. Jahresber. D. M. V., 13 (1904); and München, 1904.

258. Privorszky, A. A mathematika tanításának reformjáról. Über die Reform des Unterrichts in der Mathematik. Magyar Középiskola, 1 (1908).

259. The public schools and the reform in the teaching of mathematics (by twentytwo masters in public schools). Mathematical Gazette, 2 (1901-04): 143.

260. Queisser, F. Die Verwendung der Etymologie der termini technici im Unterrichte der Mathematik. Progr. Krumau, (1906-07), (1907-08).

261. Quossek, K. Die Realschule und die Meraner Lehrpläne. Zschr. für lateinlose höhere Schulen, 18 (1907).

262. — Die Vorschläge der Unterrichtskommission der Gesellschaft Deutscher Naturforscher und Ärzte zur Neugestaltung des mathematischen Unterrichts. Zschr. für lateinlose höhere Schulen, 17 (1906).

263. — Zu den neuen mathematischen Lehrplänen. Monatschrift für höhere Schulen, 5 (1906).

264. Rados, I. Poroszországban jelentkezö újabb törekvések a mathematikai tanitás terén. Neue preussische Reformbestrebungen im Gebiete des mathematischen Unterrichts. O. K. T. K., 38 (1905).

265. Rapport sur l'enseignement des mathématiques dans les écoles de Finlande. Rapports de la sous-commission russe, 2.

266. Die Reformbewegung im mathematischen Unterricht in Holland. Zschr. für das Realschulw., 34 (1909): 672.

267. Die Reformbewegung in Ungarn. Lietzmann's Rubrik in Zschr. math. natw. Unt., 40 (1909).

268. Réformes à accomplir dans l'enseignement des mathématiques. I. Note sur la rédaction; II. Opinion de MM. Gino Loria et Em. Borel; III. Opinion de MM. J. Andrade, David Eugene Smith, F. Marotte. L'Ens. Math., 7 (1905): 257.

269. Reformtörekvések. Über die deutschen und französischen Reformbestrebungen. Középisk. Math. Lapok, 15 (1907).

270. Reformtörekvések. Über die ungarischen Reformbestrebungen. Középisk. Math. Lapok, 15 (1907); 17 (1909).

271. Reitmann, E. Referat über Mathematik im Wiener Verein “Die Realschule." Österr. Mittelschule, 22 (1909). 
272. Report of the American Commissioners of the International Commission on the Teaching of Mathematics. David Eugene Smith, William F. Osgood, J. W. A. Young. Washington, Government Printing Office, 1912. 84 p. $8^{\circ}$. (U. S. Bureau of Education. Bulletin, No. 14, 1912.)

273. Riesz, F. Reformok a math. oktatás terén. Reforme im Gebiete des mathemátischen Unterrichts. Vortrag in Löcse. O. K. T. K., 40 (1907).

274. Risposte ad alcune domande del questionario per le riforme da compiers, nell'insegnamento della matematica. A. Natucci, E. Borel, J. Andradei D. E. Smith, G. Loria, G. Garbieri, S. Pincherle, G. Ricci. Notes in Boll. Mat., 5, 1906.

275. Rohrberg, A. Beitrag zur Reform des mathematischen Unterrichts. Zschr. math. natw. Unt., 41 (1910): 95.

276. Rorer, J. T. The curriculum-present tendencies, future possibilities. Mathematics Teacher, 4 (1911-12): 1.

277. Rose, J. Sur l'enseignement des mathématiques en Belgique. L'Ens. Math., 12 (1910): 20.

278. Rühlmann, R. Über die Reformbestrebungen auf dem Gebiete des mathematischen Unterrichts. Päd. Arch., 47 (1905).

279. Saint Laguë, A. L'enseignement mathématique et l'expérimentation. Rev. de l'Ens. des Sciences, 4 (1910).

280. Saxelby, F. M. Experimental mathematics. Nature, 66 (1902).

281. Schäffer, A. Beiträge zur Reform des mathematischen Unterrichts. Progr. Realgymn. Halberstadt, 1910; 1911.

282. Scheibner, O. Die von der Reform geforderte stärkere Betonung des Logischen. Frauenbildung, 7 (1908).

283. Schimmack, R. Die Entwickelung der mathematischen Unterrichtsreform in Deutschland, 1907-1910. Klein's Abhandlungen, III, 1.

284. — Über die Gestaltung des mathematischen Unterrichts im Sinne der neueren Reformideen. Bericht über einen Vortrag von 0 . Behrendsen beim Göttinger Ferienkurs O. 1908 und über die anschliessende Diskussion. Zschr. math. natw. Unt., 39 (1908): 513.

285. - Über die Verschmelzung verschiedener Zweige des mathematischen Unterrichts. Habilitations Vortrag Göttingen. Zschr. math. natw. Unt., 42 (1911).

286. Schlesinger, J. Über die reine Sprache im mathematischen Schulbuch. Progr. Lessingsgymn., Berlin, 1904.

287. Schmid, B. Zur Neugestaltung des mathematischen Unterrichts. Päd. Arch., 48 (1.906).

288. Schmitz-Mancy, M. Bericht über die X. Hauptversammlung des Vereins zur Förderung des lateinlosen Schulwesens in Görlitz vom 28-30 September, 1907. Zschr. für lateinlose höhere Schulen, 19 (1908).

289. Schneider, F. Zur Methodik der Elementarmathematik. Stuttgart, 1908.

290. Schotten, H. 81. Versammlung deutscher Naturforscher und Ärzte, Salzburg 19-25. September, 1909. Zschr. math. natw. Unt., 41 (1910): 98.

291. — Diskussion über die Dresdener Vorschläge der Unterrichtskommission. Zschr. math. natw. Unt., 40 (1909): 209.

292. — Die Meraner Vorschläge in der Praxis des mathematischen Unterrichts. Unterrichtsblätter, 15 (1909); Zschr. math. natw. Unt., 40 (1909).

293. — Die Meraner Vorschläge und die neuere mathematische Schulliteratur. Jahresber. der Städt. Oberrealschule, Halle a. S., 1910.

294. _ Der Unterricht in der Mathematik. Zschr. math. natw. Unt., 34 (1903): 166. 
295. Schotten, H. Welche Aufgabe hat der mathematische Unterricht auf der deutschen Schule und wie passen die Lehrpläne zu dieser Aufgabe? Int. Math. Congr. in Heidelberg. Verhandl. des III. Leipzig, 1905.

296. Schülke, A. Alte und neue Ziele im mathematischen Unterricht. Blätter höh. Schulw., 25 (1908).

297. — Der mathematische Unterricht und seine Reform. Vortrag. Jahresber. der Oberrealschule auf der Burg, Königsberg i. P. (1905-06).

298. __ Ein neuer Vorschlag zur Vertiefung des mathematischen Unterrichts. Zschr. math. natw. Unt., 33 (1902): 513.

299. — Umkehr oder Fortschritt? Zschr. math. natw. Unt., 40 (1909): 65.

300. — Vereinfachungen im mathematischen Unterricht. Päd. Blätter, 31 (1902).

301. Das Schulwesen des Kantons Basel. Städt. Erziehungsdep., (1907).

302. Schulze, E. Über einige Bezeichnungen in der Schulmathematik. Zschr. math. natw. Unt., 33 (1902): 368; 34 (1903): 35.

303. Schuster, M. Bemerkungen über Inhalt und Methode des mathematischen Unterrichts. Lehrp. und Lehrg., 16 (1900): 83.

304. Schwatt, I. J. Modern tendencies in the teaching of mathematics. Mathe. matics Teacher, 1 (1908-09): 47.

305. - On the curriculum of mathematics. Mathematics Teacher, 2 (1909-10): $171 ; 3$ (1910-11): 9.

306. Schwering, K. Ist Mathematik Hexerei? Freiberg, 1909.

307. Severi, F. See Enriques.

308. Short, R. L. See Wright, H. C.

309. Siddons, A. W. See Langley, E. M.

310. Simon, M. Bericht über die Verhandlungen der mathematischen Sektion der 46. Versammlung deutscher Philologen in Strassburg. Zschr. math. natw. Unt., 33 (1903): 125.

311. _ Didaktik und Methodik des Rechnen- und Mathematik-Unterrichtes. Baumeister's Handbuch, München, 2. Aufl., 1908.

312. — Die Klein-Gutzmer'schen Vorschläge. Mit Erwiderung von Pietzker. Südwestdeutsche Schulblätter, 24 (1907).

313. — Über Mathematik. Philosophische Arbeiten herausgegeben von Cohen und Natorp, Giessen, 1908.

314. Simons, L. G. See Baker, A. L.

315. Sitzungsbericht des Prager Vereins. Österr. Mittelschule, 19 (1906): 342.

316. Slaught, H. E. Departmental conference in mathematics. School Review, 16 (1908): 89.

317. - Ideals in the teaching of mathematics. School Science and Mathematics, 5 (1905): 702.

318. - The present trend in the teaching of mathematics. School Review, 15 (1907): 690 .

319. Slocum, S. E. The rational basis of mathematical pedagogy. Science, 26 (1907).

320. — A review of current ideas in the teaching of mathematics. Science, 30 (1909).

321. Smith, D. E. The American work of the International Commission on the Teaching of Mathematics. Mathematics Teacher, 2 (1909-10):56.

322. —_ L'enseignement des mathématiques aux États-Unis. L'Ens. Math., 3 (1901): 157.

323. - How may the teaching of mathematics be made more efficient? School Science and Mathematics, 9 (1909). 
324. Smith, D. E. The International Commission on the Teaching of Mathematics. Mathematics Teacher, 2 (1909-10); American Mathematical Monthly, 17 (1910); Educational Review, 38 (1909): 507.

325. Movements in mathematical teaching. School Science and Mathematics, 5 (1905): 135.

326. The teaching of elementary mathematics. New York, 1900.

See also Réformes à accomplir, etc.; Risposte, etc., Report of the American Commissioners.

327. Smith, E. R. See Baker, A. L.

328. Snur, H. and Gottschalk, A. Über die Reformbestrebungen auf dem Gebiete des mathematischen Unterrichts mit besonderer Berücksichtigung der Vorschläge der Gesellschaft Deutscher Naturforscher und Ärzte. Westfalen, II. Direktorenkonferenz.

329. Soecknick, K. Zeichen, Benennungen, Definitionen in der Schuimathematik. Progr. Kgl. Friedrichsgymn., Kolberg, 1910.

330. Somervell, E. L. A systematic approach to mathematics. London, 1906.

331. Souls, - Sur l'emploi de la méthode expérimentale dans l'étude des sciences mathématiques. Revue des Mathématiques Spéciales, 13 (1902).

332. Sourek, A.-V. L'enseignement mathématique en Bulgarie. L'Ens. Math., 7 (1905): 257.

333. Steinbart, O. Die Durchführung der preussischen Schulreform in ganz Deutschland. Duisberg, 1904.

334. Story, w. E. The unification of mathematics in the school curriculum. School Review, 11 (1903): 832.

335. Stromeyer, C. Mathematical training. Nature, 66 (1902): 103.

336. — The reform of mathematical teaching. Nature, 62 (1900): 523.

337. Strong, C. Some difficulties of the mathematics teacher. School Science and Mathematics, 5 (1905): 349.

338. Study, E. See Verhandlung der 50. Versammlung, etc.

339. Suppantschitsch, R. Einige Fragen des mathematischen Unterrichts und seine neue Organisation in Frankreich. Zschr. für das Österr. Gymnasialw., 58 (1907).

340. Sykes, M. Radical and conservative elements in the teaching of mathematics. School Review, 13 (1905): 59.

341. Szabó, G. See Iskolaorvosok, etc.

342. Szuics, A. La réforme de l'enseignement mathématique en Hongrie. L'Ens. Math., 11 (1909).

343. Tanfi, I. Mennyiségtani oktatásunk reformjáról. Über die Reform unseres Unterrichts in der Mathematik, 2 Artikel. Magyar Tanitóképzö, 23 (1908).

344. Tannery, J. Les mathématiques dans l'enseignement. Revue de Paris, 1900.

345. - Questions pédagogiques. Rivista di Scienza, 1 (1907).

346. The teaching of elementary mathematics. Report of the committee of the Mathematical Association, 1902. London, 1903.

347. The teaching of mathematics. Nature, 66 (1902); 88 (1911-12): 147.

348. Treutlein, P. Anschauung in der Mathematik der höheren Schulen. Vortrag beim Göttinger Ferienkurs, 1909. Lietzmann's Rubrik in Zschr. math. natw. Unt., 41 (1910).

349. Uhlig, G. Zur Berichterstattung der von der Gesellschaft Deutscher Naturforscher und Ärzte eingesetzten Unterrichtskommission. Das Hum. Gymn., 17 (1906)

350. Vaes, F. J. Le mouvement de réforme de l'enseignement mathématique en Hollande. L'Ens. Math., 11 (1909): 303.

351. Vallée-Poussin, C. de la. L'objet de la démonstration mathématique et la. réalité. Bruxelles, 1908. 
352. Verbesserung des mathematischen Unterrichts in Nordamerika. Jahresber. D. M. V., 12 (1904): 348.

353. Verein deutscher Ingenieure. Die Stellungsnahme des Vereins deutscher Ingenieure zu den gegenwärtigen Fragen des Unterrichts und der Schulorganisation. Zschr. für lateinlose höhere Schulen, 18 (1907); Zschr. math. natw. Unt., 38 (1907).

354. Versammlung deutscher Philologen und Schulmänner in Basel, 1907. Verhandlungen der 49. Herausgegeben von G. Ryhiner. Leipzig, 1908.

355. Versammlung deutscher Philologen und Schulmänner in Bonn, 1908. (S. besonders die Rede von E. Study.) Blätter höh. Schulw., 1908.

356. Visnya, A. A reform életbeléptetése külföldön és nálunk. Verwirklichung der Reform im Ausland und in Ungarn. See Beke-Mikola., Sammlung, under Secondary Schools.

357. Vorschläge des von den Wiener Vereinen "Realschule" und "Mittelschule" eingesetzten Sonderausschusses. Österr. Mittelschule, 19 (1905): 298; Zschr. für das Österr. Gymnasialw., 1905.

358. Voss, A. Über das Wesen der Mathematik. Leipzig, 1908.

359. Walckling, R. Verhandlungen der mathematischen Sektion der 47. Versammlung deutscher Philologen und Schulmänner zu Halle a. d. S. Zschr. math. natw. Unt., 34 (1903): 590.

360. Walther, F. Der mathematische Unterricht nach den Meraner Lehrplänen. Päd. Blätter, 1911.

361. Ward, F. I. The teaching of mathematics. Nature, 64 (1901): 280.

362. Weber, H. Über die Stellung der Elementarmathematik in der mathematischen Wissenschaft. Zschr. math. natw. Unt., 34 (1903).

363. Weiss, F. Wissenschaftliche Strenge im mathematischen Unterricht. Unterrichtsblätter, 8 (1902).

364. Wendler, A. Gedanken eines Mathematikers über die auf der XXI. Generalversammlung eingebrachten Änderungsvorschiäge zum mathematischen Lehrplan. Blätter für das Gymn. Schulw., 37 (1901): 577.

365. Wernicke, A. Mathematik und philosophische Propädeutik. Klein's Abhandlungen, III, 7 .

366. Wieleitner, H. Mathematische Unterrichtsfragen auf dem IV. Internationalen Mathematischen Congress. Progr. Gymn. Pirmasens, 1910; Zsschr. math. natw. Unt., 41 (1910).

367. Wimperis, H. E. The reform of mathematical education. Engineering, 1903.

368. Witting, A. Die sächsischen Fachkreise und die Reform des mathematischen Unterrichts. Unterrichtsblätter, 12 (1906).

369. — Zur Reform des mathematischen Unterrichts. Das Hum. Gymn., 16 (1905): 151.

370. Woolen, H. The reform of mathematical teaching. Nature, 62 (1900): 436 .

371. Wright, H. C. and Short, R. L. A discussion of the report of the committee on the unification of mathematics. School Science and Mathematics, 10 (1910).

372. Young, J. W. A. The movement for reform in the teaching of mathematics in Prussia. Bulletin of the American Mathematical Society, 12 (1906).

373. — My struggle with the Italian language and the morals I drew from it for the teaching of mathematics. School Review, 15 (1907): 225.

374. _ _ Die Reformbewegung im mathematischen Unterricht in den Vereinigten Staaten von Nordamerika. Jahresber. D. M. V., 15 (1906).

375. — Some recent French views on concrete methods of teaching mathematics. School Review, 13 (1905): 275.

376. - What is the laboratory method? School Science, 1903. See also Secondary Schools, and Report of the American Commissioners. 
377. Young, W. H. The introduction of the mathematical idea of infinity. Mathematical Gazette, 4 (1907).

378. Zdelar, M. Bemerkungen über die Figuren der mathematischen Lehrbücher. Zschr. math. natw. Unt., 39 (1908): 449.

379. Zur Reform des mathematischen Unterrichtes. Beschlüsse der mathematischen Sektion in Abhandl. der natw. Ges. Isis in Dresden, 1904.

380. Zweger, M. Studien im Gebiete der Elementarmathematik. Progr. Würzburg, 1905.

\section{MATHEMATICS IN THE ELEMENTARY SCHOOLS.}

381. Appell, P. Réflexions sur l'ensiegnement des mathématiques à l'école primaire. Manuel Général de l'Instruction Primaire, 44 (1908).

382. Ballard, P. B. The teaching of mathematics in London public elementary schools. Reports of the British Subcommission, 3.

383. Bioche, Ch. Enseignement primaire. Rapports de la sous-commission française, 1.

384. Bittenbinder, $\mathbf{K}$. Der mathematische Unterricht in den Volksschulen. Berichte der ungarischen Subkommission.

385. Brun, F. Om matematiker och matematikundervisningen i vära elementarskolar. Ped. Tidskr., 44 (1908).

386. Cramer, H. . See Treutlein, P.

387. Crawford, L. Does the work of the grades prepare for high-school mathematics? School Science and Mathematics, 5 (1905): 23.

388. Czuber, E. See Kraus, $\mathbf{K}$.

389. Dahlgren, H. Die Mathematik an den Volksschulen und Volkschullehrerseminaren. Berichte der schwedischen Subkommission.

390. Dressler, H. Der mathematische Unterricht an den Volksschulen und Lehrerbildungsanstalten in Sachsen und Thüringen. Klein's Abhandlungen, V, 4.

391. Gale, A. S. Teaching of mathematics in the elementary and secondary schools. Mathematics Teacher, 1 (1气08-09): 113, 121.

392. Gehrig, H. Methodik des Volks- und Mittelschulunterrichts. 2. Band. Mathematisch-naturwissenschaftliche Fächer. Leipzig, 1906.

393. Hensing, Geck and Bock. See Treutlein, P.

394. Instructions et programmes de l'enseignement scientifique dans les écoles primaires supérieures. Rev. de l'Ens. des Sciences, 4 (1910).

395. Kendall, C. N. See Mathematics in the elementary schools, etc.

396. Kerschensteiner, G. See Treutlein, P.

397. Kraus, $\mathbf{K}$. Der mathematische Unterricht an den Volks- und Bürgerschulen. (Mit einem Begleitwort von E. Czuber.) Berichte über den mathematischen Unterricht in Österreich, 1.

398. Lietzmann, W. Die Organisation der Volksschulen, gehobenen Volksschulen, Präparandenanstalten, Seminare usw. in Preussen. Klein's Abhandlungen, $\mathrm{V}, 6$.

399. McMurry, F. M. See Smith, D. E.

400. Mathematics in the elementary schools of the United States. International Commission on the Teaching of Mathematics. American report. Committees nos. I and II. C. N. Kendall, and G. D. Strayer, chairmen. Washington, D. C., 1911. 185 p. $8^{\circ}$. (U. S. Bureau of Education. Bulletin, 1911, No. 15.)

401. Nya skolstadgan för elementarlärnverken, 1904.

402. S., B. L'enseignement mathématique dans les écoles primaires et les écoles normales. Rapports de la sous-commission russe, 4 . 
403. Smith, D. E. and McMurry, F. M. Mathematics in the elementary schools. Teachers' College Record, 4 (1903).

404. Spencer, H. J. The teaching of elementary mathematics in English public elementary schools. Report of the British Subcommission, 4.

405. Stephens, I. The teaching of mathematics to young children. Reports of the British Subcommission, 11.

406. Stöcklin, J. Der mathematische Unterricht an den schweizerischen Primarschulen. Rapports de la sous-commission suisse, 2.

407. Strayer, G. $\mathbf{D}$. See Mathematics in the elementary schools, etc.

408. Tanfi, I. A népiskolai mennyiségtan tanitás módszere. Methodik des mathematischen Unterrichts der Volkschule; für die Schüler der IV. Klasse der Lehrerseminare. Budapest, Lampel, 1906.

409. Treutlein, P. Der mathematischen Unterricht an den Volksschulen und Lehrerbildungsanstalten in Süddeutschland, mit Ausführungen von $G$. Kerschensteiner und $A$. Bock über Bayern, E. Geck über Württemberg, H. Cramer über Baden, H. Hensing über Hessen. Klein's Abhandlungen, V, 3.

410. Umlauf, K. Der mathematische Unterricht an den Seminaren und Volksschulen der Hansestädte. Klein's Abhandlungen, V, 5.

411. Wines, L. D. See Secondary Schools.

412. Young, J. W. A. The teaching of mathematics in the elementary and secondary schools. New York, 1907.

413. Zaremba, S. Bericht über die speziellen Verhältnisse des öffentlichen Mathematikunterrichtes an den Volks- und Mittelschulen Galiziens. Berichte über den mathematischen Unterricht in Österreich, 8.

\section{MATHEMATICS IN THE SECONDARY SCHOOLS.}

414. Alasia, C. L'enseignement mathématique dans les premières années des lycées italiens. Réunion de Pise, avril 1909. L'Ens. Math., 11 (1909): 397.

415. Albrich, K., jr. Eingehender Lehrplan für den Mathematik-Unterricht am Gymnasium auf Grund des vom Landeskonsistorium imJahre 1909 herausgegebenen Lehrplanes. Nagyszeben, 1909.

416. - Ziel und Methode des mathematischen Unterrichts in der Mittelschule. Nagyszeben, 1905.

417. Allen,F. What mathematical equipment should the high-school graduate have? School Science and Mathematics, 8 (1908): 10.

418. American Mathematical Society. Report of committee on definition of college entrance requirements in mathematics. Bulletin of the American Math. Society (1903); Educational Review, 26 (1903): 305.

419. Amieux, Mlle. Enseignement des jeunes filles. Rapports de la sous-commission française, 5 .

420. Amtliche Protokolle der Mittelschulenquête 1908. Wien, 1908.

421. Arrêté du 25 Juillet 1911, fixant le programme de la mathématique en une de la seconde partie du baccalauréat dans les classes de mathématiques A. et B. Rev. de l'Ens. des Sciences, 5 (1911).

422. Babb, M. J. What in high-school mathematics is of most importance as a preparation for analytic geometry and the calculus in college? Mathematics Teacher, 4 (1911-12): 65.

423. Badertscher, - - Der mathematische Unterricht an den schweizerischen Sekundarschulen. Rapport de la sous-cominission suisse, 2.

424. Bass, W. The historical argument for teaching arithmetic, algebra, and geometry together in the first year of the high school. School Science and Mathematics, 5 (1905): 712. 
425. Baud, —. La classe de mathématiques élémentaires. Rev. de l'Ens. des Sciences, 5 (1911).

426. Beke, M. and Mikola, S. A középiskolai mathematikai tanitás reformja. Reform des mathematischen Unterrichts an höheren Schulen; Budapest, 1909; German edition published by Teubner, 1910, under the title: Sammlung von Abhandlungen über die Reform des mathematischen Unterrichts in Ungarn; see Lietzmann's Rubrik, in Zschr. math. natw. Unt., 1911, Kraus in the Zschr. für das Realschulw., 1912, and Loria in Mathesis, 1912. See also Beke, Der mathematische Unterricht an den Mittelschulen, in the Berichte der ungarischen Subkommission.

427. Bergmann, F. Der mathematische Unterricht an den Realschulen. Berichte über den mathematischen Unterricht in Österreich, 1.

428. Bericht über den IX. deutsch-österreichischen Mittelschultag, mathematische Sektion. Österr. Mittelschule (1906): 254.

429. Bernstein, J. Über den mathematischen und naturwissenschaftlichen Unterricht in den höheren Realanstalten. Zschr. math. natw. Unt., 33 (1902): 592.

430. Bestimmungen über die Neuordnung des höheren Mädchenschulwesens in Preussen, nebst den Bestimmungen zur zulassung der Frauen zum Universitätstudium. Zentralblatt für die gesamte Unterrichtsverwaltung in Preussen, 1910.

431. Bestimmungen über die Neuordnung des Mittelschulwesens in Preussen vom 3. Februar 1910. Berlin, 1910, and Zentralblatt für die gesamte Unterrichtsverwaltung in Preussen, 1910.

432. Bettazzi, R. Un essai de réforme des études moyennes classiques en Italie. L'Ens. Math., 7 (1905): 400.

433. Bioche, Ch. Enseignement secondaire. Rapports de la sous-commission française, 2.

434. Blutel, E. Sur l'enseignement des mathématiques dans la classe de philosophie. 2 articles. Rev. de l'Ens. des Sciences, 2 (1908).

435. Bobynin, V. L'enseignement mathématique en Russie; état actuel; enseignement secondaire. L'Ens. Math., 5 (1903): 237.

436. Bode, P. Die baierischen Oberrealschulen und ihre Lehrpläne. Zschr. für lateinlose höhere Schulen, 19 (1908).

437. Bohle, G. Die Reformbestrebungen im mathematischen Unterricht und die neuesten Lehrpläne und Lehraufgaben für die höheren Schulen in Preussen. Zschr. für lateinlose höhere Schulen, 13 (1901).

438. Bonnesen, T. Matematikken i Gymnasiet. Nyt Tidsskrift, 16 (1905).

439. Borel, E。 Les exercices pratiques de mathématiques dans l'enseignement secondaire. Conf. du Musće Pédag., 1904.

440. Böttcher, L. L'enseignement des mathématiques dans les écoles secondaires. (Polish), in Wsechseviat Warszawa, 26 (1907).

441. - Quelques remarques du sujet de la réforme de l'enseignement des mathématiques aux collèges et aux lycées. (Polish), Muzeum Lwow, 23 (1907).

442. Böttger, A. See Thaer, A.

443. Böttger, W. Amerikanisches Hochschulwesen. Leipzig, 1906.

444. Bourlet, C. Ia pénétration des mathématiques pures et des mathématiques appliquées dans l'enseignement secondaire. Conférence à Bruxelles. L'Ens. Math., 10 (1910).

445. Brandenberger, K. Der mathematische Unterricht an den schweizerischen Gymnasien. Rapports de la sous-commission suisse, 4.

446. Brookman, T. IIigh-school mathematics. School Review, 18 (1910): 20. 
447. Brown, E. E. Secondary education [in the United States]. Albany, N. Y., 1900. 65 p. $8^{\circ}$. (Monographs on education in the United States, ed. by N. M. Butler. 4.)

This monograph was contributed to the United States educational exhibit, Paris Exposition, 1900 , by the State of New York.

448. Brown, F. B. Mathematics in secondary schools. School Review, 8 (1900): 292.

449. Burger, Ch. Mathematics in the high school. Investigations of the Department of Psychological Education of the University of Colorado, 2 (1905).

450. Burján, K. A középiskolai mathematikai oktatás fejlesztési iránya. Die Entwickelungsrichtung des mathematischen Unterrichts an höheren Schulen. Progr. des äuss. Obergymn. des VII. Bez. in Budapest, 1905-06.

451. Carver, W. B. Some topics of school mathematics of special importance to those students who expect to study analytic geometry and calculus. Nathematics Teacher, 4 (1911-12): 21.

452. Circulaire du conseil scolaire de la Basse-Autriche aux directeurs des gymnases et des écoles réales. L'Ens. Math., 9 (1907): 325.

453. Clarke, J. B. Vital questions for teachers of secondary mathematics. School Science and Mathematics, 6 (1906): 561, 657.

454. Combet, E. Rapport sur l'étude, faite par le groupe parisien de l'Association des Professeurs de Mathématiques dans les classes de mathématiques A et B. Rev. de l'Ens. des Sciences, 5 (1911).

455. Comstock, C. E. The unification of secondary mathematics. National Education Association of the United States. Journal of proceedings and addresses, 1904. p. 515.

456. Conti, A. La recente riforma della scuola classica. Boll. Mat., 3 (1904).

457. Corte, D. Versuch einer freieren Géstaltung des Unterrichts in Prima; darin über den mathematischen Lehrplan von R. Fuchs. Progr. Bismarck. Gymn. Wilmersdorf, 1907.

458. Cramer, F. Die freie Behandlung des Lehrplanes auf der Oberstufe höherer Lehranstalten. Berlin, 1907.

459. Cramer, H. Der mathematische Unterricht an den höheren Schulen nach' Organisation, Lehrstoff und Lehrverfahren und die Ausbildung der Lehramts-. kandidaten im Grossherzogtum Baden. Klein's Abhandlungen, II, 4.

460. Csallóközi, J. Útmutató a gimnaziumi gyakorlati tanitásban. Wegweiser für Probekandidaten an höheren Schulen im praktischen Unterricht. Miskolcz, 1909.

461. Daninger, J. Über die Reform des mathematischen Unterrichts an der Mittelschule. Österr. Mittelschule, 20 (1906); 23 (1909).

462. Dietz, C. Aufgaben, Ziele, Berechtigungen der Oberrealschule in Bremen. Bremen, 1907.

463. Dintzl, E. Der mathematische Unterricht an den Gymnasien. Berichte über den mathematischen Unterricht in Österreich, 3.

464. — Eine vergleichende Studie über die mathematischen Lehrpläne für die österreichischen und die preussischen Realgymnasien. Zschr. für das Österr. Gymnasialw., 1909.

465. Ditzel, H. Die Mathematik in der höheren Mädchenschule und die Berechtigungsfrage. Die höhere Mädchenschule, 23 (1910).

466. Dumas, H. Les mathématiques dans la section E. Rev. de l'Ens. des Sciences, 3 (1909).

467. Ebner, F. Zur Reform des mathematischen Unterrichts an den Mittelschulen: Unterrichtsblätter, 17 (1911).

468. Erdmann, R. Die neuen preussischen Lehrpläne für das höhere Mädchenschulwesen. Die Lehrerin in Schule und Haus, 25 (1909). 
469. Erlass vom 20. März 1909, betreffend einen neuen Lehrplan für die Gymnasien. Verordnungsblatt für den Dienstbereich des Ministeriums für Kultus und Unterricht. (Österreich.)

470. Erlass vom 8. August 1908, betreffend die Errichtung von achtklassigen Realgymnasien und Reformgymnasien. Verordnungsblatt für den Dienstbereich des Ministeriums für Kultus und Unterricht. (Österreich.)

471. Erlass vom 29. Februar 1908, betreffend neue Vorschriften für die Reifeprüfungen an Gymnasien und Realgymnasien. Verordnungsblatt für den Dienstbereich des Ministeriums für Kultus und Unterricht. (Österreich.)

472. Erlass vom 23. Februar 1900. Neuauflage des Lehrplanes und der Instructionen für den Unterricht an den Gymnasien. Verordnungsblatt für den Dienstbereich des Ministeriums für Kultus und Unterricht. (Österreich.)

473. Evans, G. W. The teaching of high-school mathematics. Boston, New York, and Chicago [ $\left.{ }^{\mathrm{c}} 1911\right]$. ix, 93p. $12^{\circ}$.

474. Faragó, A. Reformok a mathematikai oktatás terén az osztrák reáliskolában. Reformbestrebungen im Gebiete des mathematischen Unterrichtes an den österreichischen Realschulen. Magyar Középiskola, 3 (1910).

475. Fehr, H. Les leçons d'introduction et les leçons de révision dans l'enseignement secondaire. L'Ens. Math., 3 (1901): 317.

476. Les mathématiques dans l'enseignement secondaire en Suisse. Congr. Int. dei Mat. Atti del IV. Roma, 1909. L'Ens. Math., 10 (1908): 285.

477. Ficquet, M. Rapport sur un projet de programmes de mathématiques pour les lycées de jeunes filles. Rev. de l'Ens. des Sciences, 5 (1911).

478. Flatt, R. Der Unterricht im Freien auf der höheren Schulstufe. Frauenfeld, 1908.

479. Freiere Gestaltung (Die) des Unterrichtes in der Prima des Lyceums zu Hannover. Monatschrift für höhere Schulen, 8 (1909); Zschr. math. natw. Unt., 40 (1909), in Lietzmann's Rubrik.

480. French, J. S. College entrance examinations in mathematics. Educational Review, 27 (1904): 305.

481. - The practical limitations of an ideal course in secondary mathematics, and the educational waste or economy in the proposed sequence of studies. National Education Association of the United States. Journal of proceedings and addresses, 1910. p. 511.

482. Freud, Ph. Die mathematischen Schulbücher an den Mittelschulen und verwandten Anstalten. Berichte über den mathematischen Unterricht in Österreich, 6.

483. Füger, J. Schulordnung für die humanistischen Gymnasien, Progymnasien und Lateinschulen im Königreich Bayern. Würzburg, 1900. (Enthält auch die Prüfungsordnungen für das höhere Lehramt.)

484. Gale, A. S. What in high school mathematics is of most importance as a preparation for analytic geometry and the calculus in college? Mathematics Teacher, 4 (1911-12): 58.

See also Elementary Schools.

485. Gallucci, G. L' insegnamento della matematica nelle scuole medie. Il Pitagora (Palermo), 9 (1902-03).

486. - I programmi di matematica per le scuole classiche. Boll. Mat., 1 (1902).

487. Geck, E. Der mathematische Unterricht an den höheren Schulen nach Organisation, Lehrstoff und Lehrverfahren und die Ausbildung der Lehramtskandidaten im Königreich Württemberg. Klein's Abhandlungen, II, 3:

488. Gehrig, H. See Elementary Schools.

489. Gercken, W. Das mathematische Lehrpensum für die Prima der Realanstalten. Blätter höh. Schulw., 21 (1904).

490. Geschichte der oberen Realschule in Basel. Basel (1905). 
491: Geuther, N. See Thaer, A.

492. Giebel, K. Über mathematischen Unterricht an den höheren Schulen. Referat über die Vorlesung von F. Klein im W. S. 1904-5. Math. Natw. Blätter, 1905.

493. Goller, - - Der Lehrplan der baierischen Realgymnasien. Bay. Zschr. für Realschulw., 15 (1908).

494. Göransson, E. Bericht über die eingegangenen Antworten auf eine Umfrage über den mathematischen Unterricht an Real- und gemischten Schulen. Berichte der schwedischen Subkommission.

495. _ Die Mathematik an den schwedischen Gymnasien. Berichte der schwedischen Subkommission.

See also Hallgren, E.

496. Götting, E. Darlegung der Grundsätze für den mathematischen Unterricht am Göttinger Gymnasium. Vortrag am Göttinger Ferienkurs, 1909. Zschr. math. natw. Unt., 41 (1910), in Lietzmann's Rubrik.

497. — Über das Lehrziel im mathematischen Unterricht der höheren Lehranstalten. Jahresber. D. M. V., 11 (1902); Zschr. math. natw. Unt., 33 (1902): 294; wieder abgedruckt in Klein-Riecke, Neue Beiträge. (Under Correlation.)

498. Graham, E. Practical suggestions as to ways of improving the product of the high school. School Science and Mathematics, 9 (1909).

499: Gray, J. C. A general science course of elementary physics and mathematics combined. School Science and Mathematics, 12 (1912): 377.

500. Gubler, S. E. Der mathematische Unterricht an den höheren Mädchenschulen der Schweiz. Rapports de la sous-commission suisse, 3.

501. Gwatkin, E. R. The value of the study of mathematics in public secondary schools for girls. Reports of the British Subcommission, 18.

502. A gymnasiumi tanitás terve és a reá vonatkozó utasitások. Lehrplan und Instructionen des Unterrichtes an Gymnasien; neue Ausgabe der Instructionen vom Jahre 1903. Budapest.

503. Haeseler, O. Mathematik und Reformgymnasium. Zschr. für die Reform der höheren Schulen, 20 (1909).

504. Hallgren, E. and Görannson, E. Mathematik an den schwedischen Realschulen. Berichte der schwedischen Subkommission; and Ped. Tidskr., 50 (1910).

505. Hasse, W. Beiträge zur Methodik des mathematischen Unterrichtes in den unteren und mittleren Klassen. Progr. städt. Kaiser-Friedrich Realgymn., Rixdorf, 1909.

506. Hecht, C. Über Notwendigkeit und Gestaltung des Unterrichts in der elementaren Mathematik an den höheren Mädchenschulen. Vortrag. Bielefeld, 1905.

507. Höfler, A. Didaktik des mathematischen Unterrichts. Erster Band der didaktischen Handbücher für den realistischen Unterricht an höheren Schulen, herausgegeben von A. Höfler und Fr. Poske. Leipzig, 1910.

508. — Drei Vorträge zur Mittelschulreform. Wien, 1908.

509. __ Gymnasium. Encyklopädisches Handbuch der Erziehungskunde, p. 628-645.

510. — Vorschläge zu einer zeitgemässen Umgestaltung des mathematischen Unterrichtes an den österreichischen Gymnasien und Realschulen, im Auftrage der deutschen Mittelschule in Prag erstattet. Zschr. math. natw. Unt., 37 (1906): 145; Bericht hierüber in Österr. Mittelschule (1906): 342.

511. Horn, E. Das höhere Schulwesen der Staaten Europa's (Stundenpläne). II. Aufl., Berlin, 1907.

512. Hornig, —. Die sächsische Oberrealschule. Zschr. für lateinlose höhere Schulen, 19 (1908). 
513. Hossfeld, C. Der mathematische Unterricht an den höheren Schulen Thüringens. Klein's Abhandlungen, II, 6.

514. Hume, A. Mathematics in preparatory schools. School Review, 8 (1900): 75.

515. Instructionen für den Unterricht an den Realschulen in Österreich im Anschluss an einen Normallehrplan. Wien.

516. Istruzioni e programmi vigenti nei ginnasi e licei colle modificazioni a tutto il 24 ottobre 1900 . Torino, 1905.

1517. Klein, F. Hundert Jahre mathematischen Unterrichts an den höheren preussischen Schulen. In Lexis, Die Reform des höheren Schulwesens in Preussen. Halle, 1902; Klein-Riecke, Neue Beiträge, etc.; Jahresber. D. M. V., 13 (1904).

518. — Über den mathematischen Unterricht an den höheren Schulen. Jahresber. D. M. V., 11 (1902); Zschr. math. natw. Unt., 33 (1902): 114; Klein-Riecke, Neue Beiträge, etc.

519. — Über eine zeitgemässe Umgestaltung des mathematischen Unterrichts an höheren Schulen. Leipzig, Teubner, 1904; reprinted in Klein-Riecke, Neue Beiträge (under Correlation), etc.

520. - and Schimmack, R. Vorträge über den mathematischen Unterricht an den höheren Schulen. Leipzig, 1907.

521. Kleinpeter, H. Die Hauptrichtung der Reformtätigkeit unserer Mittelschulen. Vortrag; s. hierzu die Antwort von A. Höfler. Österr. Mittelschule, 20 (1906): 289.

522. ——ZurReform des realistischen Unterrichtsam Gymnasium. Zschr.für das Österr. Gymnasialw. (1902): 807.

523. Kondratiev, V. L'enseignement mathématique dans les gymnases de garçons du Ministère de l'Instruction publique et dans les instituts de jeunes filles du ressort des établissements de l'Impératrice Marie. Rapports de la souscommission russe, 4 .

524. Konrath, Th. Der mathematische Unterricht an den Mädchenlyzeen. Berichte über den mathematischen Unterricht in Österreich, 4.

525. Krause, M. Über die Reformbestrebungen auf dem Gebiete des mathematischen Unterrichts auf höheren Schulen seit 1890. Abhandl. der natw. Ges. "Isis" in Dresden, 1904.

526. Kretzschmar, J. F. Das höhere Schulwesen im Königreich Sachsen. Leipzig, 1903.

527. Krollik, H. Die Neugestaltung des französischen Unterrichtswesens im XX. Jahrhundert, insbesondere auf dem Gebiete der höheren Schulen. Zschr. für lateinlose höhere Schulen, 18 (1907).

528. Lehrpläne:

Baden. See Württemberg.

Bayern. Lehrplan der bayerischen Oberrealschulen. Ministerialblatt für Kirchen- und Schulangelegenheiten im Königreich Bayern; auch separat erschienen bei Kestner und Callweg, München.

Crefeld. Lehrplan der Oberrealschule zu Crefeld. Programm, 1909.

Elberfeld. Lehrpläne der Reformabteilung und der nach Fachgruppen geteilten Primen des städtischen Realgymnasiums zu Elberfeld. Programm, 1909.

Görlitz. Lehrplanentwurf des Gymnasiums Augustum zu Görlitz. Programm, 1906.

Hamburg. Lehrpläne und Lehrgänge für die Oberrealschulen des Staates Hamburg. 1904.

See also Württemberg. 
528. Lehrpläne-Continued.

Kiel. Lehrpläne und Lehraufgaben der Oberrealschule an der Waitzstrasse in Kiel. (S. hier den officiellen Lehrplan für den Unterricht in der Mathematik, der auf Grund der Meraner Entwürfe ausgearbeitet wurde.) Beilage zum Jahresbereichte, 1906.

Magdeburg. Lehrpläne für den Unterricht in Rechnen, Mathematik, etc., der Guericke-Oberrealschule in Magdeburg. Jahresberichte, 1909.

Österreich. Lehrpläne und Instructionen für den Unterricht an den Gymnasien in Österreich. Wien, 1904.

- Lehrpläne der österreichischen Gymnasien und Realschulen vom Jahre 1909, Wien, 1909. See also Lietzmann's Rubrik in Zschr. math. natw. Unt., 41 (1910),

Sachsen. Lehrpläne der sächsischen Oberrealschulen. Gesetz und Verordnungsblatt für das Königreich Sachsen (1908): 81; auch bei Meinhold, Dresden separat erschienen.

Schönberg. Lehrplan der Hohenzollernschule in Schönberg bei Berlin. See Lietzmann's Rubrik in Zschr. math. natw. Unt., 40 (1909).

Wilmersdorf. Lehrplan des Pismarckgymnasiums in Wilmersdorf. Programm, 1907.

Württemberg. Lehŗplan für die Oberrealschulen in Württemberg, Baden, und Hamburg. Zschr. für lateinlose höhere Schulen, 17 (1906).

- Die neuen Lehrpläne für die höheren Lehranstalten Württembergs (vom 19. vii-25. viii 1904). Zschr. für lateinlose höhere Schulen, 17 (1906).

529. Lietzmann, W. Die Organisation des mathematischen Unterrichts an den höheren Knabenschulen Preussens. Klein's Abhandlungen, I, 2.

530. — Stoff und Methode des mathematischen Unterrichts der höheren Schulen Deutschlands. Taschenbuch für Mathematik und Physik, II, Leipzig, 1911.

531. — Stoff und Methode im mathematischen Unterricht der norddeutschen höheren Schulen. Auf Grund der vorhandenen Lehrbücher. Klein's Abhandlungen, I, 1.

532. Liman, O. Inwieweit ist eine Umgestaltung des mathematischen Unterrichts an den höheren Lehranstalten im Sinne der Meraner Vorschläge zu empfehlen? Hauptbericht, mit einem Mitbericht von J. Schjerning, 77. Direktorenversammlung, Posen, 1907; Berlin, 1907.

533. Lindemann, F. See Waldvogel, J.

534. Lobky, B. A középiskolai mathematikai tanitás reformja. Reform des mathematischen Unterrichts an höheren Schulen. Jahrbuch des 14. Kongr. der röm. kath. Prof. in Siebenbürgen, Kolozsvár, 1910.

535. Lorey, W. Freiere Gestaltung und Privatstudien im mathematischen Unterricht der oberen Klassen. Zschr. math. natw. Unt., 39 (1908): 73.

536. — - Staatsprüfung und praktische Ausbildung der Mathematiker an den höheren Schulen in Preussen und einigen norddeutschen Staaten. Klein's Abhandlungen, I, 3.

537. Loria, G. La riforma della scuola media in Germania. Rivista Ligure, Genova, 1906.

538. — La scuola media e la sua attuale crisi di sviluppo. Padova, 1909.

539. Málly, F. Olasz középiskolai reformáramlatok. Reformbestrebungen der italienischen höheren Schulen. O. K. T. K., 41 (1907).

540. Marotte, F. Les récentes réformes de l'enseignement des mathématiques dans l'enseignement secondaire français. Jahresber. D. M. V., 13 (1904).

541. Maierialien zu einem Lehrprogramm der bayerischen Qberrealschulen. Bay. Zschr. für Realschulw., 15 (1908). 
542. Mathematics in the public and private secondary schools of the United States. International Commission on the Teaching of Mathematics. American report. Committees nos. III and IV. G. W. Evans and W. E. Stark, chairmen. Washington, D. C., 1911. 187 p. $8^{\bullet}$. (U. S. Bureau of Education. Bulletin, 1911, no. 16.)

543. Meyer, E. Die Einführung der Mathematik in die höhere Mädchenschule. Frauenbildung, 2 (1903).

544. Meyer, Fr. Die neuen Lehrpläne für die höheren Lehranstalten Württembergs. Zschr. für lateinlose höhere Schulen, 17 (1906).

545. Mikola, S. See Beke, M.

546. Milthaler, J. Der mathematische Lehrstoff der Oberstufe. Lehrp. und Lehrg., 1909.

547. Modifications apportées au plan d'étude des lycées et collèges de garçons. L'Ens. Math., 8 (1906): 65 .

548. Mollberg, - - Der mathematische Unterricht in der höheren Mädchenschule. Jahresberichte des Sophienstifts in Weimar, 1903.

549. Most, S. Der mathematische Unterrichtsstoff und das mathematische Bildungsgebiet in den oberen Klassen des Realgymnasiums und der Oberrealschule. Wissenschaftliche Beilage zum Jahresber. des Realgymn. zu Coblenz, 1901.

550. Müller, H. Das höhere Schulwesen Deutschlands am Anfang des XX. Jahrhunderts. Stuttgart, 1904.

551. — Die Mathematik auf den Gymnasien und Realschulen. Leipzig, 1906.

552. Myers, G. W. Correlation of the subjects in secondary mathematical teaching. School Review, 11 (1903): 21.

553. - Early impressions of the teaching of mathematics in the secondary schools of Paris. School Science and Mathematics, 11 (1911).

554. — Mathematics at University High School. School Review, 14 (1906): 57.

555. — The year's progress in the mathematical work of the University High School. School Review, 15 (1907): 576.

556. Nannei, E. Cause del poco profitto che fanno nello studio della matematica i giovanni delle scuole medie. Congr. fra i prof. di mat. delle scuole medie italiane promosso dall' Assoc. Mathesis. Atti del iii. Torino, 1909.

557. Nath, M. Die Bildungsaufgabe der Mathematik im Lehrplan der höheren Schulen. Unterrichtsblätter, 1904; Berlin, 1904.

558. — Die Gestaltung der Lehrpläne an den höheren Schulen bei Annahme der von der Unterrichts-Commission der deutschen Naturforscherversammlung gemachten Vorschläge. Neue Jahrb., 9 (1906).

559. — Lehrpläne und Prüfungsordnungen im höheren Schulwesen Preussens seit Einführung des Abiturienten-Examens. Berlin, 1900.

560. — Die preussischen Lehrpläne für den mathematischen Unterricht am Gymnasium und die Vorschläge der Breslauer Unterrichtscommission. Jahresber. D. M. V., 15 (1906).

561. - Zur Frage des mathematischen Lehrpensums in der Prima der realistischen Lehranstalten. Zschr. für lateinlose höhere Schulen, 18 (1907).

562. Natucci, A. Discussione sui programmi della scuola media riformata. Boll. Mat., 7 (1908).

563. Neuberg, J. Rapport sur l'enseignement secondaire des mathématiques. (Germany, Austria,'Hungary, Holland, Italy, Sweden, Luxembourg.) Bruxelles, 1911.

564. Die Neuordnung der höheren Mädchenschulen in Sachsen. Lietzmann's Rubrik, Zschr. math. natw. Unt., 42 (1911).

565. Die Neuordnung des Mittelschulwesens in Preussen. Zschr. math. natw. Unt., $41(1910)$. 
566. Newbold, w. Higher mathematics for the classical sixth form. Reports of the British Subcommission, 1.

567. Newhall, C. W. Correlation of mathematical studies in secondary schools. National educational association of the United States. Journal of proceedings and addresses, 1903. p. 480 .

568. — Discussion. A high school mathematics club. Educational Review, 29 (1905): 515.

569. - The real problem in secondary mathematics. Educational Review, 43 (1912): 472.

570. Noble, C. A. The teaching of mathematics in California secondary schools. School Science and Mathematics, 7 (1907): 762.

571. Nodnagel, __. Das höhere Schulwesen im Grossherzogtum Hessen. 1903.

572. Noodt, G. Über die Stellung der Mathematik im Lehrplan der höheren Mädchenschule vor und nach der Neuordnung des höheren Mädchenschulwesens in Preussen. Zschr. math. natw. Unt., 40 (1909): 185.

573. — Wie ist auf höheren Mädchenschulen das Interesse für den mathematischen Unterricht zu wecken? Frauenbildung, 5 (1906).

574. Nuovi programmi di matematica per i ginnasi ed $\mathrm{i}$ licei approvati per R. D. 11. nov. 1904. Boll. Mat., 3 (1904).

575. Nya lärnverksstadgan jamte instruktion for ofverstyrelsen. Stockholm, herausgegeben von Berggvist.

576. Oblath, R. A mathematika tanítása a vii. osztályban. Der Unterricht in Mathematik in der vii. Klasse. Gyakorlati Paedagogia, 3 (1907).

577. Organisation und Lehrplan der künftigen Oberrealschule in Bayern. Zschr. phys. chem. Unt., 20 (1907).

578. Packard, J. C. Suggestions as to a course in mathematics for the entering class in high school. School Science and Mathematics, 6 (1906): 292.

579. Palotay, A. A középiskolai mathematikai tanitás reformjáról. Über die Reform des mathematischen Unterrichts der höheren Schule. Progr. Staatl. Oberrealschule Arad, 1910.

580. Plan d'études et programmes de l'enseignement dans les lycées et collèges. Paris.

581. Plan d'études et programmes de l'enseignement secondaire. Paris, 1902.

582. Polakoff, S. Die Mathematik und die Mittelschule. (Russian) Mosk. Päd. Sammlung, 1907.

583. Pradel, C. Communication du groupe de Dijon de l'Association des professeurs de mathématiques sur la question de l'allègement du programme de mathématiques dans les classes de mathématiques A et B. Rev. de l'Ens. des Sciences, 5 (1911).

584. Die preussischen Lehrpläne für die höheren Mädchenschulen. Zschr. phys. chem. Unt., 32 (1909).

585. Programmes des examens du nouveau baccalauréat de l'enseignement secondaire. Paris, 1902.

586. Projet de programme pour la classe de mathématiques spéciales, publié par la Revue des mathématiques spéciales. L'Ens. Math., 7 (1905): 148.

587. Quossek, K. Das mathematische Lehrpensum für die Prima der Realanstalten. Zschr. für lateinlose höhere Schulen, 15 (1904).

588. La recente riforma degli studi secondari in Francia del 30 maggio, 1902. Periodico di Matematica, 3 (1906).

589. Regulations of the Oxford and Cambridge school-examination board. Various years.

590. Reiff, R. Vorschlag zu einem mathematischen Lehrplan für die Gymnasien. Korrespondenzblatt, 6 (1906).

$47398^{\circ}-12-3$ 
591. La réorganisation de l'enseignement mathématique dans les écoles supérieures de jeunes filles en Prusse. (Nach Arbeiten von Noodt und Lorey.) L'Ens. Math., 12 (1900).

592. Report on the teaching of mathematics in preparatory schools. Mathematical Gazette, 4 (1907-08): 137.

593. Réunion des maîtres de mathématiques des écoles moyennes autrichiennes. L'Ens. Math., 7 (1905): 145.

594. Richter, A. Ein Abschluss der Reform des mathematischen Gymnasialunterrichts durch die preussische Unterrichtskommission. Zschr. math. natw. Unt., 32 (1901).

595. — Die Reform des mathematischen Gymnasialunterrichts durch die Unterrichtskommission der Gesellschaft Deutscher Naturforscher und Ärzte. Zschr. math. natw. Unt., 37 (1906): 141.

596. Rönstrom, A. and Josephson, O. Die Mathematik an den höheren Mädchenschulen in Schweden. Berichte der schwedischen Subkommission, and Ped. Tidskr., 14 (1910).

597. Schjerning, J. See Liman, O.

598. Schlesser, E. La classe de mathématiques élémentaires. Rev. de l'Ens. des Sciences, 5 (1911).

599. Schlosser, A. Tetschener vereinigte Mittelschule. 7. Jahresber. Städt. Ober Realgymn. Tetschen i. E. (1905-06).

600. Schnell, H. Der mathematische Unterricht an den höheren Schulen und die Ausbildung der Lehramtscandidaten im Grossherzogtum Hessen. Klein's Abhandlungen, II, 5 .

601. Schröder, J. Die neuzeitliche Entwicklung des mathematischen Unterrichts an den höheren Mädchenschulen, insbesondere in Norddeutschland. Klein's Abhandlungen, I, 5 .

602. - Verhandlungen beim Göttinger Ferienkursus (Ostern 1906) über die Reform des mathematischen Unterrichts an den höheren Schulen. Zschr. math. natw. Unt., 37 (1906): 563.

603. Schülke, A. Über die Reform des mathematischen Unterrichts an höheren Schulen. Zschr. math. natw. Unt., 37 (1906): 161.

604. Schwatt, I. J. Is the average secondary school pupil able to acquire a knowledge of all the mathematics ordinarily given in the school? Mathematics Teacher, 3 (1910-11): 101.

605. Schwering, K. Zur Methodik des mathematischen Unterrichts am Gymnasium. Zschr. math. natw. Unt., 33 (1902): 26.

606. Shobinger, J. J. On the teaching of secondary mathematics. School Review, 15 (1907): 83 .

607. — Practical suggestions as to ways of improving the mathematical output of secondary schools. School Science and Mathematics, 5 (1905): 401.

608. Short, R. L. Secondary mathematics. School Science and Mathematics, 7 (1907): 290.

609. - The seventh to tenth grades a unit in mathematics. Mathematics. Teacher, 1 (1908-09): 150 .

610. Simon, O. L'enseignement des mathématiques au gymnase au trichien. L'Ens. Math., 4 (1902): 157.

611. Sintsof, D. Sur les dernières modifications dans l'enseignement des mathématiques aux écoles réales en Russie. L'Ens. Math., 11 (1909); Lietzmann's Rubrik in 'Zschr. math. natw. Unt., 40 (1909).

612. Skinner, E. B. The arrangement of the mathematical studies in the highschool course-a comparison with the French system. School Science and Mathematics, 10 (1910). 
613. Smith, D. E. L'enseignement mathématique dans les écoles secondaires aux Etats-Unis. L'Ens. Math., 10 (1908): 269.

See also The teaching of mathematics in the secondary schools of the United States. (Congr. Int. Mat. Atti del IV. Roma, 1909); and Reports of the American Commission, Elementary Schools, Geometry.

614. Stark, W. E. The teaching of mathematics in the private secondary schools of the United States of America. School Science and Mathematics, 11 (1911).

615. Story, $\mathbf{L}$. The organization of the teaching of mathematics in public secondary schools for girls. Reports of the British Subcommission, 9.

616. Stscherbina, $\mathbf{K}$. Die Mathematik in den russischen Mittelschulen. Kiev Universität, 11 (1908).

Russian report on the development of these schools in the period 1899-1907.

617. Suppantschitsch, R. L'application des idées modernes des mathématiques à l'enseignement secondaire en Autriche. Congr. Int. dei Mat. Atti del IV. Roma, 1909.

618. Szabó, P. A mathematika tanitása a gyakorló fögymnasiumban. Mathematischer Unterricht am Übungsgymnasium. Progr. des Übungsgymn. Budapest, 1911; Berichte der ungarischen Subkommission.

619. Szenes, A. A középiskolai mathematikai tanitás reformja. Reform des mathematischen Unterrichts an höheren Schulen auf Grund der ungarischen Bestrebungen. Polgárisk. Közl., 13 (1909).

620. Thaer, A. Lehrplanentwurf für die Oberrealschule vor dem Holstenthor zu Hamburg, 1902-03.

621. — Die Mathematik als Unterrichtsgegenstand der höheren Mädchenschulen. Zschr. für Weibliche Bildung, 29.

622. - Geuther, N. and Böttger, A. Der mathematische Unterricht in den Gymnasien und Realanstalten der.Hansestädte Mecklenburgs und Oldenburgs. Klein's Abhandlungen, I, 4.

623. Tinsley, S. B. What is the least amount of mathematics that should be in any secondary school course? National Education Association of the United States. Journal of proceedings and addresses, 1904. p. 514.

624. Tyler, H. W. Report of the committee on entrance requirements in mathematics. National Education Association of the United States. Journal of proceedings and addresses, 1903. p. 481. Also published in the Bulletin of the American Math. Society.

625. Undervisningsplan för gymnasiet, 1909, iv. 30. Svensk Förfättrings-Samling, Nr. 28, 1909.

626. Undervisningsplan för realskolan, 1906. Stockholm, edited by Berggvist; see also Svensk Forfättnings-Samling, Nr. 10, 1906.

627. Vailati, G. L'insegnamento della matematica: a) nel primo triennio della scuola secondaria; b) nello studio inferiore della scuola secondaria. Boll. Mat., 5 (1906).

628. — La matematica nell'insegnamento secondario. Boll. Mat., 6 (1907).

629. _ Sugli attuali programmi per l' insegnamento della matematica nelle scuole secondarie italiane. Congr. Int. dei Mat. Atti del IV. Roma, 1909.

630. __ Sull'insegnamento della matematica nello studio superiore della scuola secondaria. Boll. Mat., 6 (1907).

631. Verein deutscher Ingenieure. Aussprüche des Ausschusses für Hochschulund Unterrichtsfragen. Zschr. des Ver. deutscher Ing., 50 (1906).

632. Veronese, G. Interpellanza sulla recente riforma di programmi nelle scuole classiche del regno, 1905, iii. 4. Roma, 1905.

633. Versammlung zur Beratung von Hochschul- und Unterrichtsfragen. Zschr. math. natw. Unt., 38 (1907); Zschr. des Ver. deutscher Ing., 51 (1907). 
634. Verslag van het verhandelde ter 41 ste algemeene vergadering van de vereeniging van leeraren aan inrichtingen van middelbaar onderwijs. Alkmaar, 28-30 Aug., 1906. (A.) Sectie voor Wiskunde. Referat von G. J. van de Well, Weekblad for Gymn. en Midd. Ond., 3 (1907).

635. Visnza, A. Der mathematische Unterricht an den höheren Töchterschulen. Berichte der ungarischen Subkommission.

636. Vogt, H. Mathematik und Reformgymnasium. Leipzig, 1907.

637. Vogt, K. W. Bericht über den mathematischen Unterricht an den russischen Realschulen. Rapports de la souscommission russe, 3.

638. Volenszky, Gy. A magyarországi polgárískolák mathematikai oktatásának állapota. Referat über den mathematischen Unterricht der ungarischen Bürgerschulen. Polgáriskolai Közlöny, 15 (1911). Berichte der ungarischen Subkommission.

639. Vuibert, H. La réforme de l'enseignement secondaire. Paris, 1902.

See also his later work, La réforme de l'enseignement secondaire expliquée aux familles. Paris, 1907.

640. Wahlgren, A. Om kursena i matematik på latingymnasiet. Ped. Tidskr., 4 (1905).

641. Waldvogel, J. Die Gymnasialmathematik in der Beleuchtung des Herrn Prof. Dr. Lindemann. Blätter für das Gymn. Schulw., 41 (1905): 50.

642. Walsh, C. B. Mathematics in Ethical Culture High School. Mathematics Teacher, 2 (1909-10).

643. Well, G. J. van de. See Verslag, etc.

644. Wieleitner, H. Der mathematische Unterricht an den höheren Lehranstalten sowie die Fortbildung der Lehrkräfte im Königreich Bayern. Klein's Abhandlungen, II, 1.

645. Wilk, E. Bemerkungen zu dem Lehrplan in Mathematik für die höheren Schulen in Preussen vom Jahre 1901. Jahresbericht des Vereins für wissenschaftliche Pädagogik, Dresden, 35 (1901).

646. Wines, L. D. Mathematics in and below the high school. School Science and Mathematics, 7 (1907): 183.

647. Wirz, J. Der mathematische Unterricht an den höheren Knabenschulen sowie die Ausbildung der Lehramtskandidaten in Elsass-Lothringen. Klein's Abhandlungen, II, 7.

648. Witting, A. Der mathematische Unterricht an den Gymnasien und Realanstalten, nach Organisation, Lehrstoff und Lehrverfahren und die Ausbildung der Lehramtskandidaten im Königreich Sachsen. Klein's Abhandlungen, II, 2.

649. Wottling, A. Rapport sur l'étude faite par les professeurs de l'Académie de Lille pour alléger le programme des classes de mathématiques A. et $B$. Rev. de l'Ens. des Sciences, 5 (1911).

650. Wychgram, J. Der neue mathematische Lehrplan der höheren Töchterschulen in Frankreich. Frauenbildung, 10 (1911).

651. Young, J. W. A. Current tendencies in secondary mathematics in France. School Science and Mathematics, 7 (1907): 489.

652. — Current tendencies in secondary mathematics in Italy. School Science and Mathematics, 7 (1907): 352.

653. - The teaching of elementary mathematics in the higher schools of Prussia. New York, 1911.

See also Reports of the American Commission; Elementary Schools.

654. Zaremba, S. See Elementary Schools.

655. Ziehen, J. Über die bisherige Entwickelung und die weiteren Aufgaben der Reform unseres höheren Schulwesens. Frankfurt, 1908. 


\section{MATHEMATICS IN THE TECHNICAL SCHOOLS.}

656. Adamicka, M. Der mathematische Unterricht an der höheren Forstlehranstalt Reichstadt. Berichte über den mathematischen Unterricht in Österreich, 2.

657. Arany, D. Der mathematische Unterricht an den höheren Gewerbeschulen und gewerblichen Fachschulen.- Berichte der ungarischen Subkommission.

658. Arbeiten auf dem Gebiete des technischen Mittelschulwesens. Abhandlungen und Berichte über technisches Schulwesen, Leipzig, 1910.

659. Ashford, C. E. See Mercer, J. W.

660. Chatelier, H. L'enseignement technique dans ses rapports avec l'enseignement universitaire. Rev. de l'Ens. des Sciences, 3 (1909); Revue de Métallurgie, 1908.

661. Crelier, I. Les mathématiques dans l'enseignement technique moyen en Suisse. Rapports de la sous-commission suisse, 5 .

662. Czuber, E. Der mathematische Unterricht an den technischen Hochschulen. Berichte über den Mathematischen Unterricht in Österreich, 5.

663. Dolinski, M. Der mathematische und physikalische Unterricht an den höheren Handelsschulen. Berichte über den Math. Unt. in Österreich, 2.

664. Ehrig, G. Über Stoff und Methode des mathematischen Unterrichts an Baugewerkschulen. Leipzig, 1905.

665. Erikson, G. Die Mathematik an der technischen Schule in Stockholm. Berichte der schwedischen Subkommission, 1911.

666. Frixon,H. L'enseignement professionelle en Allemagne. Revue Pédagogique, 1 (1908).

667. Furtwängler, P. Die mathematische Ausbildung der Feldmesser. Klein's Abhandlungen, IV, 8.

668. Gallander, O. See Koch, H. von.

669. Girndt, M. Der mathematische Unterricht an den Baugewerkschulen. Klein's Abhandlungen, IV, 3.

670. Goldziher, K. Kereskedelmi és gazdasági ismeretek tanítása a középiskolában. Der Unterricht in der Handels- und Wirtschaftslehre auf der höheren Schule. Kereskedelmi Szakoktatás, 16 (1907).

671. Grossmann, M. Der mathematische Unterricht an der Eidgenössischen Technischen Hochschule. Berichte der schweizerischen Subkommission, 7.

672. Grübaum, H. Der mathematische Unterricht an den deutschen mittleren Fachschulen der Maschinenindustrie. Klein's Abhandlungen, IV, 1.

See also his article entitled, Der mathematische Unterricht an einem Technikum, Zschr. math. natw., Unt., 40 (1909): 424; Unterrichtsblätter, 15 (1909).

673. Haese, - Die mathematischen Fächer an den gewerblichen Fortbildungsschulen. Klein's Abhandlungen, IV, 5.

674. Hagström, K. I. Die Mathematik an elementartechnischen Gewerbeschulen. Berichte der schwedischen Subkommission, 1911.

675. Haskins, C. N. See Mathematics in the technical secondary schools, etc.

676. Havas, M. and Bogyó, S. Der mathematische Unterricht an den Handelsschulen. Berichte der ungarischen Subkommission.

677. Heumann, C. Die Mathematik in den Abteilungen der Maschinen-und Baugewerkschulen an den elementartechnischen Gewerbeschulen. Berichte der schwedischen Subkommission.

678. Huntington, E. V. The two-hour system at Harvard. See Mathematics in the technological schools of collegiate grade in the United States, p. 40.

679. Jahnke, E. Die Mathematik an Hochschulen für besondere Fachgebiete. Klein's Abhandlungen, IV, 7.

680. Kârmán, T. Részletek az angol müszaki oktatás köréböl, Perry irányáról. Einzelheiten über den technischen Unterricht in England, Perry'sche Richtung. Magyar Mérnök és Épitész-Egylet Heti Értesitöje, 31 (1908). 
681. Klein, F. See Colleges and Universities.

682. Kobalt, E. Der mathematische Unterricht an den Montanistischen Hochschulen. Berichte über den mathematischen Unterricht in Österreich, 10.

683. Koch, H. von and Gallander, O. Die Mathematik an den schwedischen technischen Lehranstalten. Berichte der schwedischen Subkommission.

684. Lacombe, M. L'enseignement mathématique à l'école d'ingénieurs de Lausanne. Rapports de la sous-commission suisse, 8.

685. Laisant, C. A. Les nouveaux programmes de l'École Polytechnique de Paris. L'Ens. Math., 5 (1903): 77.

686. Lazzeri, G. L'insegnamento della matematica nella R. Accademia Navale di Livorno e nella R. Accademia di Torino. Atti della Sottocommissione Italiana.

687. Lehrpläne der Handelsschule (Oberrealschule) in Bremen. 1904.

688. Low, D. A. Geometry for Engineers. Reports of the British Subcommission, 8. 689. Maestro, T. Proposta per una riforma dei programmi di matematica nelle scuole techniche feminili. Boll. Mat., 4 (1905).

690. Mathematics at West Point and Annapolis. International Commission on the Teaching of Mathematics. American report. Committee no. XI. C. B. Upton, chairman. Washington, D. C., 1912. 25 p. $8^{\circ}$ (U. S. Bureau of Education. Bulletin, 1912, no. 2.)

691. Mathematics in the technical secondary schools in the United States. International Commission on the Teaching of Mathematics. American report. Committee no. VI. C. N. Haskins, chairman. Washington, D. C., 1912. (U. S. Bureau of Education. Bulletin, 1912, no. 4.)

692. Mathematics in the technological schools of collegiate grade in the United States. International Commission on the Teaching of Mathematics. American report. Committee no. IX. H. W. Tyler, chairman. Washington, D. C., 1911. (U. S. Bureau of Education. Bulletin, 1911, no. 9.)

693. Matter, $\mathbf{K}$. Organisation und Methodik des mathematischen Unterrichts in den Landerziehungsheimen. Berichte der schweizerischen Subkommission, 3 .

694. Meldau, H. See Schilling, C.

695. Mercer, J. W. and Ashford, C. E. Mathematics at Osborne and Dartmouth. Reports of the British Subcommission, 17.

696. Mikuta, A. Der mathematische Unterricht an den k. u. k. Militär-Erziehungsund Bildungsanstalten. Berichte über den mathematischen Unterricht in Österreich, 10.

697. Morf, L. Les mathématiques dans l'enseignement commercial suisse. 'Rapports de la sous-commission suisse, 1912.

698. Ott, C. Die angewandte Mathematik an den technischen Mittelschulen der Maschinenindustrie. Klein's Abhandlungen, IV, 2.

699. Penndorf, B. Die Mathematik an den kaufmännischen Lehranstalten. Klein's Abhandlungen, IV., 6.

700. Perry, J. The preliminary education of the engineer. School Science, 2 (1902): 264.

701. Poprugenko, M. L'enseignement des mathématiques dans les Corps de Cadets. Rapports de la sous-commission russe, 5.

702. Possê, C. See Colleges and Universities.

703. Rados, G. Der heutige Stand des mathematischen Unterrichts am königlichungarischen Josefs-Polytechnikum. (Technische Hochschule in Budapest.) lierichte der ungarische Subkommission.

704. Reich, K. Der mathematische Unterricht an dem k. k. Technologischen Gewerbemuseum in Wien. Berichte über den mathematischen Unterricht in $\ddot{\text { sterreich, } 10 .}$ 
705. Richter, A. Die Studenten der Mathematik auf den technischen Hochschulen. Zschr. math. natw. Unt., 34 (1903): 473.

706. - Der Verein deutscher Ingenieure und der Besuch der technischen Hochschulen durch die Studenten der Mathematik. Zschr. math. natw. Unt., 40 (1909): 207.

707. Rollet, P. Enseignement technique. Rapports de la souscommission française, 4 .

708. Rulf, W. Der mathematische Unterricht an den gewerblichen Lehranstalten. Berichte über den mathematischen Unterricht in Österreich, 4.

709. Schilling, C. and Meldau, H. Die Mathematik an den Seefahrtsschulen. Klein's Abhandlungen, IV, 4.

710. Scorza, G. L'insegnamento della matematica nelle scuole e negli istituti tecnici. Atti della Sottocommissione Italiana.

711. Scott, C. F. The teaching of mathematics for engineers. Science, 28 (1908): 129.

712. Simony, O. Der mathematische Unterricht an der k. k. Hochschule für Bodenkultur in Wien. Berichte über den mathematischen Unterricht in Österreich, 10.

713. Stäckel, P. Die mathematische Ausbildung der Architekten, Chemiker, und Ingenieure an den deutschen technischen Hochschulen. Klein's Abhandlungen, IV, 9.

714. Symposium of mathematics for engineering students. (Chicago.) Science, 28 (1908): 129, 134, 288.

715. Tyler, H. W. The Chicago symposium on mathematics for engineering students. Bulletin of the American Mathematical Society, 15 (1909). See also Mathematics in technical schools of collegiate grade.

716. Upton, C. B. See Mathematics at West Point and Annapolis.

717. Usherwood, T. S. Mathematics with relation to engineering work in the schools. Reports of the British Subcommission, 12.

718. Woodward, R. S. The point of view in teaching engineering mathematics. Science, 28 (1908): 134. See also Reports of the British Subcommission, 12.

\section{MATHEMATICS IN THE COLLEGES AND UNIVERSITIES.}

719. Ball, w. W. Rouse. The Cambridge school of mathematics. Math. Gazette, 6 (1912).

720. Bestimmungen zur Einladung der Frauen zum Universitätstudium. Zentralblatt für die gesamte Lnterrichtsverwaltung in Preussen, 1910.

721. Blutel, E. La réforme des programmes d'admission aux grandes écoles en 1904. Rev. de l'Ens. des Sciences, 1 (1907); 2 (1908).

722. Bobynin, V. L'enseignement mathématique en Russie; état actuel; enseignement supérieur. L'Ens. Math., 5 (1903): 397.

723. Bôcher, M. See Graduate work in mathematics, infra.

724. Borel, E. Enseignement des mathématiques dans les facultés. Congrès de l'Association française pour l'avancement des sciences, 1908.

725. Buhl, A. L'enseignement dans les universités populaires. L'Ens. Math., 4 (1902): 37.

726. Chatelier, H. See Technical Schools.

727. Directions générales concernant les plans des études mathématiques à l'Université de Genève. Suisse Universitaire, 7 (1901).

728. Enriques, F. L'Università italiana. Revista di Scienza "Scientia," 3(1906).

729. Escherich, G. V. Reformirage unserer Universitäten. Jahresber. D. M. V., 13 (1907). 
730. Graduate work in mathematics in universities and in other institutions of like grade in the United States. International Commission on the Teaching of Mathematics. American report. Committee no. XII. M. Bôcher, chairman. Washington, D. C., 1911. (U. S. Bureau of Education. Bulletin, 1911, No. 6.)

A lso in Builetin of the American Mathematical Society, 17 (1910-11).

731. Graf, J. H. Der mathematische Unterricht an den schweizerischen Universitäten. Rapport de la sous-commission suisse, 8 .

732. Greenstreet, W. J. See Macaulay, F. S.

733. Gutzmer, A. Über die auf die Anwendungen gerichteten Bestrebungen im mathematischen Unterricht der deutschen Universitäten. Jahresber. D. M. V., 13 (1907).

734. Hatzidakis, N. J. Sur l'état actuel des mathématiques supérieures en Grèce. L'Ens. Math., 3 (1901): 397.

735. Hawkes, H. E. Mathematics in the college course. Educational Review, 40 (1910): 145.

736. Klein, F. De l'enseignement des sciences mathématiques et physiques dans les universités et hautes écoles techniques. L'Ens. Math., 8 (1906): 5.

737. — Über die Aufgaben und die Zukunft der philosophischen Fakultät. Rede, Göttingen 1909. Abgedruckt in Jahresber. D. M. V., 13 (1909).

738. Lilienthal, R. von. Ratschläge und Unterweisungen für die Studierenden der Mathematik und Physik an der Universität Münster. Jahresber. D. M. V., 15 (1906).

Similar instructions have been published for students at Göttingen, Jena, Kiel, and Leipzig.

739. Lorey, W. Das Studium der Mathematik an den deutschen Universitäten seit 1870. Klein's Abhandlungen, III, 8.

740. Macaulay, F. S. and Greenstreet, W. J. Examinations for mathematical scholarships. Reports of the British Subcommission, 14.

741. Mathematical reform at Cambridge. Nature, 68 (1903): 178.

742. Pincherle, S. Sugli studi per la laurea in matematica e sulla sezione di matematica delle scuole di magisterio. Atti della Sottocommissione Italiana.

743. Possé, C. Rapport sur l'enseignement mathématique dans les universités, les écoles techniques supérieures, et quelques-unes des écoles militaires. Rapports de la sous-commission russe, 1 .

744. Saint-Germain, A. de. Enseignement supérieure. Rapports de la souscommission française, 3; L'Ens. Math., 12 (1910): 177.

745. Sedgwick, Mrs. H. Higher mathematics for women. Reports of the British Subcommission, 18.

746. Snow, L. F. The college curriculum in the United States. Dissertation, Columbia University, New York, 1907.

747. Somigliana, C. Intorno all'ordinamento degli studi matematici nel primo biennio universitario in Italia. Atti della Sottocommissione Italiana.

748. Sterneck, R. von. Der mathematische Unterricht an den Universitäten. Berichte über den mathematischen Unterricht in Österreich, 7.

749. Townsend, E.J. An analysis of the failures in Freshman mathematics. School Review, 10 (1902): 675.

750. Undergraduate work in mathematics in colleges of liberal arts and universities. International Commission on the Teaching of Mathematics. American report. Committee no. X. H. S. White, chairman. Washington, D. C., 1911. 30 p. $8^{\circ}$. (U. S. Bureau of Education. Bulletin, 1911, no. 7. )

751. White, H. S. See Undergraduate work in mathematics, etc.

752. Wilcynski, E. J. Mathematical appointments in colleges and universities. Bulletin of the American Mathematical Society, 15 (1909). 
753. Wilson, W. H. To what extent is a closer correlation of the different branches of college mathematics desirable from the teacher's standpoint? School Science and Mathematics, 5 (1905): 235.

754. Wiman, A. Die Mathematik an den schwedischen Universitäten. Berichte der schwedischen Subkommission; Ped. Tidskr., 1910.

See also Technical Schools and Correlation.

\section{THE TEACHING OF ARITHMETIC.}

755. Adhémar, R. See Montessus, R.

756. Amaddii, I. L'insegnamento dell'aritmetica nelle scuole elementari. Firenze, 1907.

757. Atmanspacher, O. Der Rechenunterricht im ersten Schuljahre. Leipzig, 1906.

758. Babb, M. J. Some remarks on approximate computation. Mathematics Teacher, 3 (1910-11): 43.

759. Baewert, —. See Ziegler, —.

760. Bailey, M. A. The teaching of arithmetic in elementary schools. National Education Association of the United States. Journal of proceedings and addresses, 1905 . p. 380 .

761. Bandini, S. Sull'insegnamento dell'aritmetica e dell'algebra nella scuola inferiore. Boll. Mat., 8 (1909).

762. Beke, M. Vezérkönyv a népiskolai számtani oktatáshoz. Leitfaden zum Rechenunterricht der Volksschule. Budapest, 1900.

763. Berdellé, C. Propédeutique du calcul. L'Ens. Math., 6 (1904): 440.

764. Bertola, G. L'insegnamento dell'aritmetica nella scuola elementare. Conferenza.

765. Borchardt, P. Angewandtes Rechnen in der Sexta. Essen, 1901.

766. Branford, B. A study of mathematical education including the teaching of arithmetic. Oxford, 1908.

767. Brocke, E. See Algebra.

768. Brown, J. C. An investigation on the value of drill-work in the fundamental operations of arithmetic. Journal of Educational Psychology, 2 (1911).

769. Browne, C. E. Psychology of the simple arithmetical processes. American Journal of Psychology, 17 (1906): 1.

770. Brucher, K. Anschauung in der Arithmetik. Bamberg, 1911; Zschr. math. natw. Unt., 42 (1911).

771. Bruns, P. Zur Vorbereitung des mathematischen Unterrichts durch den Rechenunterricht. Jahresber. Realschule Pleschen, 1910.

772. Bühler, - Neue Wege im Rechenunterricht. Korrespondenzblatt, 14 (1907): 382, 428.

773. Büttner, A. Anleitung zum Rechenunterricht in der Volksschule. Leipzig, 1903.

774. Cailler, C. Une leçon sur la théorie élémentaire des fractions. L'Ens. Math., 6 (1904): 25.

775. Chiari, A. L'insegnamento dell'aritmetica e della geometria nelle scuole elementari. Boll. Mat., 5 (1907).

776. Ciamberlini, C. Sull'insegnamento dell'aritmetica e della geometria nelle scuole primarie. Bologna, 1902.

777. Collins, J. V. Discussion. The superintendent and the course in arithmetic. Educational Review, 27 (1904): 83.

778. Cosamassima, M. Sull'insegnamento dell'aritmetica pratica nelle scuole secondarie inferiore. Boll. Mat., 1 (1902).

779. Csada, I. A röviditett szorzás tanitásához. Zum Unterricht der abgekürzten Multiplication. Nevelés, 27 (1910). 
780. Csada, I. A röviditett szorzás hibája. Fehler bei der abgekürzten Multiplication. Magyar Tanitóképzö, 27 (1912).

781. Darnet, L. Counting and adding. American Journal of Psychology, 16 (1905): 327 .

782. Deckars, R. Die Veranschaulichung der Grundoperationen im Rechenunterricht. Neue Bahnen, 14 (1903).

783. Discussion of the arithmetic syllabus in secondary schools. Mathematical Gazette, 6 (1911): 32 .

784. Doerge, O. See Geometry.

785. Dooley, W. H. Arithmetic in a Massachusetts industrial school. School Science and Mathematics, 11 (1911).

786. Durrell, C. V. The arithmetic syllabus in secondary schools. Mathematical Gazette, 6 (1911): 28.

787. Epps, F. A first practical training in arithmetic. Mathematical Gazette, 4 (1907).

788. Färber, C. Über die Reformbestrebungen im arithmetisch-algebraischen Unterricht an höheren Schulen. Sitzungsber. der Berliner Math. Gesellschaft, 9 (1910); Zschr. math. natw. Unt., 40 (1909).

789. Fazzari, G. Nuova notazione nei problemi dell'aritmetica pratica. Il Pitagora Palermo, 13 (1906-07).

790. Fick, E. Zum Arithmetikunterricht. Blätter für das Gymn. Schulw., 37 (1901): 544 .

791. Frischauf, J. Zum Rechnen mit unvollständigen Zahlen. Zschr. für das Realschulw., 32 (1907).

792. Fröhlich, K. Az alsófokú számtani tanitás célszerü berendezéséröl. Über die zweckmässige Gestaltung des Rechenunterrichtes der Unterstufe. See Beke-Mikola, Sammlung, under Secondary Schools.

793. Gauss, A. Einige Grundbegriffe der Arithmetik, ihr Zusammenhang und ihre geometrische Deutung. Festschr. Dom- und Realgymn. Kolberg, 1908.

794. Geiger, K. Die Neunerprobe in der zweckmässigsten Form. Blätter für das Gymu. Schulw., 44 (1908): 480.

795. Gerlach, A. Der Rechenunterricht auf der Elementarstufe. Päd. Jahrb., 1911.

796. Göbelbecker, L. F. Das rechnerische Sachprincip in seiner historischen Entwickelung. Wiesbaden, 1901.

797. Goldziher, K. A számtan tanításának tárgyi köre:. Sachgebiete des Rechenunterrichts. O. K. T. K., 39 (1906).

798. — Der Rechenunterricht auf der Unterstufe der höheren Schulen. Zschr. math. natw. Unt., 39 (1908): 289.

799. Grimm, O. Die Einführung in die Percentrechnung. Archiv für Schulpraxis, Paderborn, 1900.

800. Guyou, E. Note sur les approximations numériques. Paris, 1909.

801. Haacke, C. Entwurf eines arithmetischen Lehrganges für höhere Schulen. Leipzig, 1905.

802. Hart, H. F. Highest common factor. Mathematics Teacher, 3 (1910-11): 167.

803. Hartmann, B. Der Rechenunterricht in den deutschen Volksschulen vom Standpunkt des erziehenden Unterrichts. Leipzig, 1904.

80-1. Hedgepeth, V. W. B. Spelling and arithmetic in 1846 and to-day. School Revicw, 14 (1906): 352.

805. Hedrick, E. R. Approximations and approximation processes. School Science and Mathematics, 8 (1908).

806. Heininger, Rais, Weit and Zenhain. Das Sachrechnen. Cannstadt, 1904.

807. Hiemesch, R. H. Präparationen für den Rechenunterricht. 1903. 
808. Hočvar, F. Über den arithmetischen Unterricht im Obergymnasium. Zschr. für das Österr. Gymnasialw., 52 (1901).

809. Huguenard, E. Lettre d'un professeur de physique sur l'enseignement du calcul. Rev. de l'Ens. des Sciences, 1 (1907).

810. Jackson, C. S. The elementary arithmetic of the theory of numbers. Mathematical Gazette, 4 (1908).

811. Jackson, L. L. The educational significance of sixteenth-century arithmetic. New York, 1906.

812. Jahn, O. Einiges vom Zahlbegriff. Progr. Städt. Gymn. Halle a. S., 1905.

813. Juckel, Gy. A korlátolt pontosságú müveletek hibái. Fehler bei den aborekürzten Operationen. Kereskedelmi Szakoktatás, 18 (1909-10).

814. — Numerikus kifejezések megközelitö kiszámitása. Approximative Berechnung numerischer Ausdrücke. Kereskedelmi Szakoktatás, 19 (1910-11).

815. Killing, W. and Hovestadt, H. See General topics.

816. Kirchberger, R. Zur Behandlung der gemeinen Brüche auf der untersten Stufe der Mittelschule. Zschr. für das Realschulw., 30 (1905): 337.

817. Kolhtsch, A. Was veranschaulichen wir im Rechenunterricht? Zschr. Lehrmittelwesen und päd. Literatur, 1908.

818. Kraus, K. Rechenunterricht in den Volks- und Bürgerschulen. Encyklopädisches Handbuch der Erziehungskunde, von Dr. J. Loos, Wien.

819. Kühn, F. Zur Methodik des Rechenunterrichts in der Quarta der höheren Lehranstalten. Lehrp. und Lehrg., 22 (1906): 435.

820. Lanner, A. Die wissenschaftlichen Grundlagen des ersten Rechenunterrichts. Wien, 1901.

821. Lietzmann, W. Arithmetik und Algebra in den höheren Schulen Frankreichs. Zschr. math. natw. Unt., 37 (1906): 228, 302, 399.

822. — Stoff und Methode des Rechenunterrichts in Deutschland. Ein Literaturbericht. Klein's Abhandlungen, V, 1.

823. Lightfoot, I. Approximations in arithmetic. London, Normal Press.

824. Lopič, P. J. Die Grundoperationen im Obergymnasium. Progr. Bozen (1905-06).

825. Lorenz, K. Das Rechnen mit unvollständigen Zahlen. Progr. Waidhofen a. d. Thaya (1901-02).

826. Lorey, w. Über die Wohlthat und das Werden der Zahl. Progr. Gymn. Görlitz, 1905.

827. Mc Murry, C. A. Special method in arithmetic. New York, 1907. vii, 225 p. 12 ?

828. Mehmke, R. Numerisches Rechnen. Encykl. der Math. Wissensch., I, 941.

829. Mende, J. Megjegyzések Fröhlich Károlynak "Az alsófokú számtani tanitás célszerü berendezéséröl irt cikkéhez. Bemerkungen zum Artikel K. Fröhlich's "Über die zweckmässige Gestaltung des Rechenunterrichts der Unterstufe." O. K. T. K., 48 (1910).

830. Messineo-Cantarella, S. L'insegnamento dell'aritmetica nelle scuole elementare. Acireole, 1908.

831. Mikola, S. A kutató és mérö módszer az alsófokú számtan és mértan tanitásában. Die Beobachtungs- und Messungsmethode im Rechen- und GeometrieUnterricht der Unterstufe. O. K. T. K. 35 (1902-1903). See Beke-Mikola, Sammlung, under Secondary Schools.

832. Möller, M. Die abgekürzte Decimalbruchrechnung. Wien, 1906.

833. Montessus, R. and Adhémar, R. Calcul numérique. Encyclopédie Scientifique, Paris, 1912.

834. Morawetz, J. Über die Grundlagen der Arithmetik und Algebra im Mittelschulunterricht. Progr. K. K. Staatsrealschule Salzburg, 1904. 
835. Nath, M. Hilfsmittel zum Unterricht im Rechnen und in der Arithmetik. Monatschrift für höhere Schulen, 1909-1910. Several articles.

836. Natucci, A. La riforma nell'insegnamento dell'aritmetica. Boll. Mat., 4 (1905).

837. - Sulla necessità di coordinare l'insegnamento dell'aritmetica a quello della geometria. Boll. Mat., 8 (1909).

838. Newcomb, Simon. Methods of teaching arithmetic. Educational Review, 31 (1906): 339 .

839. - The teaching of arithmetic. National Education Association of the United States. Journal of proceedings and addresses, 1906. p. 86.

840. Nitsche, O. Der arithmetische Lehrgang im symmetrischen Aufbau. Progr. Kgl. Kais. Augusta Gymn. Charlottenburg, 1910.

841. Paetz, W. Die Procentrechnung in der Quarta höherer Lehranstalten. Lehrp. und Lehrg., 1907.

842. Palmer, G. W. The teaching of arithmetic in secondary schools. Reports of the British Subcommission, 13.

843. Pařizek, A. Zur Theorie des grössten gemeinschaftlichen Masses und des kleinsten gemeinschaftlichen Vielfachen (Bohemian). Progr. Hohenmauth (1904-05).

844. Pedoth, A. Enstehung des Begriffes der ganzen und gebrochenen Zahlen und die einfachen Rechnungsarbeiten mit diesen. Progr. Reichenberg (1905-06).

845. Rais. See Heininger.

846. Rorer, J. T. An arithmetical test in three Philadelphia high schools. Mathematics Teacher, 3 (1910-11): 185.

847. Sainte Laguë, A. Calcul pratique et approximations numériques. Rev. de L'Ens. des Sciences, 3 (1909).

848. — Sur le calcul pratique. Rev. de l'Ens. des Sciences, 3 (1909).

849. Saunders, S. A. Contracted multiplication and division. Mathematical Gazette, 4 (1907).

850. Schneider, —. Denken und Schauen im Rechnen. Päd. Arch., 53 (1911).

851. Schülke, A. Zahlenrechnungen im Unterricht. Zschr. math. natw. Unt., 43 (1912).

852. Schwacha, P. B. Rechnungsproben im Gebiete der elementaren Operationen. Progr. Wilhering (1908-09).

853. Schwarz, P. Das Wesen der Zahl. D. Bl. erzieh. Unt., 37 (1909-10).

854. Schweter, R. Methodische Bemerkungen über die unterrichtliche Behandlung und die Gruppirung des Rechenstoffes. Wittenberg, 1903.

855. Shaw, C. A. The new syllabus in arithmetic for New York State. Mathematics Teacher, 3 (1910-11): 189.

856. Smith, D. E. The outlook for arithmetic in America. Boston, 1904.

857. - The teaching of arithmetic. Teachers College Record, 10 (1909). Revised and published separately in 1911; new edition in preparation.

858. — The teaching of elementary mathematics. New York, 1901.

859. Starch, D. Transfer of training in arithmetical operations. Journal of Educational Psychology, 2 (1911).

860. Steuer, W. Methodik des Rechenunterrichts nebst einem Abriss eines Unterrichtsganges in der Raumlehre. Breslau, 1903.

861. Stitt, E. W. School and business arithmetic-limitations and improvements. National Education Association of the United States. Journal of proceedings and addresses, 1900. p. 566.

862. Stone, C. W. Arithmetical abilities and some factors determining them. Dissertation, 'Teachers College, Columbia University, New York, 1908. 
863. Streng, K. and Zuckersdorfer, J. Praktische Anleitung zur Behandlung des Rechenunterrichts in der Volksschule. Wien, 1908.

864. Suppantschitsch, R. Über die Multiplication und Division unvollständiger Zahlen. Zschr. für das Realschulw., 29 (1904): 465.

865. Suzzallo, H. The teaching of primary arithmetic. Boston, 1912. $\mathbf{x}, 123$ p. 12 .

866. Szenes, A. A számtantanítás modszeréhez. Zur Methodik des Rechenunterrichts. Polgárisk. Közl., 12 (1908).

867. Thorndike, E. L. Practice in the case of addition. American Journal of Psychology, 21 (1910): 483.

868. — Relation between memory for words and memory for numbers. American Journal of Psychology, 21 (1910): 487.

869. Torrès, L. See Graphical Representation.

870. Tripard, L. Le calcul approximatif. L'Ens. Math., 4 (1910).

871. - Méthode pratique de calcul approximatif. Rev. de l'Ens. des Sciences, 3 (1909).

See also Calcul numérique et calcul mental. Rev. de l'Ens. des sciences, 4 (1909).

872. Utescher, O. Der Rechenunterricht an höheren Lehranstalten. Progr. Oberrealschule Freiburg i. Schlesien, 1905.

873. Valiron, G. Sur les programmes d'arithmétique et d'algèbre. Rev. de l'Ens. des Sciences, 5 (1911).

874. Vatuzzi, A. La riforma dell'insegnamento dell'aritmetica razionale. Boll. Mat., 4 (1905).

875. Vereinfachtes Quadriren. Zschr. für das Realschulw., 34 (1909): 722.

876. Vollprecht, H. Das Rechnen eine Vorbereitung zur allgemeinen Arithmetik. Leipzig, 1902.

877. Wagner, L. Veranschaulichung und praktische Aufgaben im Elementarrechnen; nebst Antwort von A. Ritthaler. Neue Bahnen, 16 (1905).

878. Wallentin, J. G. Arithmetik an den höheren Lehranstalten. Enzyklopädisches Handbuch der Erziehungskunde. Band 1; 46.

879. Walsemann, H. Anschauungsmittel im Rechenunterricht und ihre Verwendung. Neue Bahnen, 16 (1905).

880. Webster, F. Comparison of the fundamental operations. School Science and Mathematics, 5 (1905): 80 .

881. Weit. See Heininger.

882. White, W. F. The decimalization of arithmetic. School Science and Mathematics, 8 (1908): 409.

883. - Teaching of the metric system. School Science, 2 (1902): 350 .

884. Wieleitner, H. Der Zahl- und Mengebegriff im Unterricht. Unterrichtsblätter, 12 (1906).

Sez also his vorks in Mathemat. Bibliothek, nos. 2 and 7, 1912.

885. Wilk, E. Neue Rechenmethode, gegrundet auf das natürliche Werden der Zahlen und des Rechnens. Päd. Psych. Studien, 29 (1908).

886. Williams, R. P. Why teachers universally favor the metric system. School Science and Mathematics, 6 (1906): 351.

887. Williams, S. Shortening the course in arithmetic. Mathematics Teacher, 2 (1909-10): 147.

888. Winch, W. H. Accuracy in school children. Does improvement in numerical accuracy transfer to arithmetical reasoning? Journal of Educational Psychology, 1 (1910): 557.

889. — Further work on numerical accuracy in school children. Does improvement in numerical accuracy transfer? Journal of Educational Psychology, 2 (1911).

890. Young, W. H. Teaching of arithmetic. Education, 39 (1909).

891. Zenhain. See Heininger. 
892. Ziegler, —. Die Stellung der Decimalbrüche. Päd. Abhandl., Bielefeld, 6 (1901).

893. - Die socialpädagogischen Beziehungen des arithmetischen Unterrichts. Päd. Abhandl., Bielefeld, 6 (1901). In derselben Sammlung über dasselbe Thema eine Abhandlung von Baewert, 7 (1902).

894. Zoll, O. Eine geometrische Begründung der Arithmetik auf der Schule. Math. Natw. Blätter, 2 (1905).

895. Zuckersdorfer, J. See Streng, K.

\section{THE TEACHING OF ALGEBRA.}

896. Ackermann, C. Solution by factoring of problems under the factor theorem. School Science and Mathematics, 6 (1906): 493.

897. Baker, A. L. Algebraic multiplication. School Science and Mathematics, 8 (1908): 493.

898. Ballard, P. B. The teaching of algebra; its connection with arithmetic. School World, 3 (1909).

899. Biklé, C. E. The aims in teaching algebra and how to attain them. Mathematics Teacher, 1 (1908): 77.

900. Board of Education. See Geometry.

901. Bozóky, E. Az algebrai egész függvényekröl és az elemi uton megfejthetö maximum-minimum feladatokról. Über algebraische ganze Funktionen und über elementare Lösung von Maximum-Minimum Aufgaben. Középisk. Math. Lapok, 8 (1901).

902. Bremiker, H. Über die Behandlung der Ungleichungen im Unterricht. Progr. Kgl. Wilhelm Gymn., Berlin, 1911.

903. Brocke, E. Über die Benutzung der Beziehungen zwischen Mengen und Zahlen im arithmetisch-algebraischen Unterricht. Progr. Gymn. Zabern (1908-09).

904. Bruës, M. Zur Proportionalitätslehre. Zschr. math. natw. Unt., 4 (1910): 555.

905. Cajori, F. Discussion of the report of the committee on algebra. School Science and Mathematics, 8 (1908): 205.

906. Capelli, A. Sull' opportunità di dare nell' insegnamento secondario una sviluppo maggiore alla matematica combinatoria. Boll. Mat., 5 (1906).

907. Central Association. Report of committee on algebra in the secondary schools. School Science and Mathematics, 7 (1907): 694.

908. Collins, J. V. Economies in algebra. School Review, 13 (1905): 324.

909. Cornish, W. A. The effort to make algebra yield fruit. Mathematics Teacher, 1 (1908-09): 155; 2 (1909-10): 1.

910. Curtis, A. M. What should be the aims in teaching algebra and how to attain them. Mathematics Teacher, 1 (1908-09): 36.

911. Danielewicz, B. Orachunek prawdopodobienstwa skole sředniej. Die Wahrscheinlichkeitsrechnung im Unterricht der höheren Schulen. Wiadomošci Matematycjne, 13 (1909).

912. Davis, E. W. Sce Rouse, E. L.

913. Dean, P. A few algebra methods. Mathematics Teacher, 2 (1909-10): 41. See also p. 81.

914. Diekmann, J. Zur Auflösung der homogenen quadratischen Gleichungen mit zwei Unbekannten. Zschr. math. natw. Unt., 35 (1904): 499.

915. A discussion of the draft report of the committee on the teaching of algebra and trigonometry. Mathematical Gazette, 6 (1911): 42.

916. Donecker, F. C. An algebraic balance. School Science and Mathematics, 5 (1905): 411. 
917. Dressler, H. Die Entwickelungsgeschichte einer angewandten Gleichungsaufgabe. Unterrichtsblätter, 11 (1905).

918. Durrell, F: Modern tendencies in the teaching of algebra. Mathematics Teacher, 1 (1908-09): 85.

919. Eckhardt, E. Elementare Ableitung der Realitätsbedingungen für die Gleichungen dritten Grades ohne Auflösung dieser Gleichungen. Zschr. math. natw. Unt., 33 (1902): 446.

920. Evans, G. W. Discussion. Algebra in elementary schools. Educational Review, 28 (1904): 305.

921. Färber, C. See Arithmetic.

922. Faulland, J. Das Radizieren. Blätter für das Gymn. Schulw., 42 (1906): 85.

923. Genese, R. W. On the teaching of indices and surds. Mathematical Gazette, $1(1896-1900): 339$.

924. Godfrey, C. The algebra syllabus in the secondary school. Reports of the British Subcommission, 5.

925. - The teaching of algebra: What is educational and what is technical? Mathematical Gazette, 1909.

926. Goldziher, K. Bemerkungen über die Behandlung der quadratischen Gleichung im elementaren algebraischen Unterricht. Unterrichtsblätter, 18 (1911).

927. — Zur Methode der Multiplication relativer Zahlen. Zschr. math. natw. Unt., 41 (1910); Wiadomości mat. 15 (1912), Polish by Z. Gubalski.

928. Goodrich, M. F. The presentation of positive and negative numbers. School Science and Mathematics, 11 (1911).

929. Greenwood, G. W. Limits in elementary algebra. School Science and Mathematics, 8 (1908): 156.

930. Grühl, P. Die Lösung der Gleichung II. Grades mit einer Unbekannten $x$ und ihre Anwendung auf Gleichungen höheren Grades. Päd. Bausteine, No. 3-4. Berlin.

931. Haidl, H. Die Näherungsmethoden für Auflösung numerischer Gleichungen. Progr. Ober Hollabrunn (1906-07).

932. Hedrick, E. R. Imaginary quantities in elementary mathematics. School Science and Mathematics, 9 (1909).

933. - On the selection of topics for elementary algebra. School Science and Mathematics, 11 (1911).

934. Janzen, O. Die komplexen Zahlen im Unterricht der höheren Lehranstalten. Zschr. math. natw. Unt., 40 (1909).

935. Jones, F. T. Individual instruction in algebra and geometry. National Education Association of the United States. Journal of proceedings and addresses, 1905. p. 466.

936. Keferstein, H. Eine gemeinsame Methode zur Lösung der Gleichungen II., III., und IV. Grades. Zschr. math. natw. Unt., 37 (1906).

937. Killing, W. and Hovestadt, H. See General topics.

938. Kiseljak, M. Eine neue Auflösungsmethode der homogenen quadratischen Gleichungen zwischen zwei Unbekannten. Zschr. math. natw. Unt., 34 (1903): 559 .

939. Kleinpeter, H. Die allgemeine Arithmetik auf der Oberstufe der Mittelschule. Zschr. für das Realschulw., 27 (1902).

940. Krug, - - Die niedere Analysis auf der Unterstufe des Realgymnasiums. Progr. Kgl. Realgymn. Stuttgart, 1902-03 and 1906.

941. Kriise, K. Die unendliche geometrische Reihe. Zschr. math. natw. Unt., 36 (1905): 161.

942. Lennes, N. J. Another algebraic balance. School Science and Mathematics, 5 (1905): 602 . 
943. Lennes, N. J. Modern tendencies in the teaching of algebra. Mathematics Teacher, 1 (1908-09): 94.

944. Lissák, J. A középiskolai számtan és algebra tanitásának reformjáról. Über die Reform des arithmetischen und algebraischen Unterrichts der höheren Schule. Jahresber. der Staatl. Obergymn. VI. Bezirk, Budapest, 1910.

945. Loomis, H. B. High school algebra. School Science and Mathematics, 7 (1907): 590 .

946. McDill, R. N. Presentation of the ideas of positive and negative numbers to beginners. School Science and Mathematics, 7 (1907): 307.

947. McKelden, A. M. Problems that arise in the teaching of elementary algebra in the first year of the high school. School Science and Mathematics, 7 (1907): 363.

948. McKinney, T. K. and Stone, J. C. The teaching of algebra in its relation to the present educational trend. National Education Association of the United States. Journal of proceedings and addresses, 1908, p. 628.

949. Marjanek, A. Cubing and the extraction of cube root with decimal numbers. (Bohemian.) Progr. Strassnitz (1908-09).

950. Millis, J. F. Some modern notions in the rational teaching of elementary algebra. School Science and Mathematics, 7 (1907): 176.

951. Missouri Society of Teachers of Mathematics and Science. Recommendations for a high-school course in algebra. School Science and Mathematics, 8 (1908).

952. Morawetz, J. See Arithmetic.

953. Myers, G. W. A class of content problems for high-school algebra. School Science and Mathematics, 7 (1907): 19; School Review, 14 (1906): 563.

954. Newhall, C. W. The teaching of algebra by the laboratory method. School Science and Mathematics, 5 (1905): 40.

955. Noodt, G. Wie lassen sich die Meraner Vorschläge über die Reform des mathematischen Unterrichts für den algebraischen Unterricht an Lyceen verwerten? Frauenbildung, 5 (1906).

956. Nordlund, K. P. Ell tilläg i den algebraiska kursen. Ped. Tidskr., 43 (1907).

957. Nunn, J. P. The aim and method of school algebra. I. The aim of algebra teaching. Mathematical Gazette, 6 (1911): 167.

958. Orbán, Gy. A másod- és harmadfokú egyenletek valós gyökeinek közelitö meghatározása parabola segitségével. Näherungsweise Berechnung der realen Wurzeln von quadratischen und kubischen Gleichungen mittels der Parabel. Középisk. Math. Lapok, 16 (1909).

959. Osborn, C. S. Thought values in beginning algebra. School Review, 10 (1902): 169 .

960. Puller, —. Elementare Behandlung der Maximum-Minimumaufgaben. Unterrichtsblätter, 10 (1904).

961. Rabes, O. Das Princip der Konvergenz im Unterricht. Zschr. für Lehrmittelwesen und päd. Literatur, 6 (1910).

962. Rietz, H. L. An exposition of the Illinois syllabus on algebra. National Education Association of the United States. Journal of proceedings and addresses, 1909, p. 511.

963. — Is the situation in regard to the teaching of algebra in our high school satisfactory? School Science and Mathematics, 8 (1908): 496.

964. Rouse, E. L., Wooley, J. F. and Davis, E. W. An outline for algebra and geometry. School Science and Mathematics, 8 (1908): 18.

965. Saposnikov, A. Krisisz szovremennahv prepodavanija algebri. Krisis des heutigen algebraischen Unterrichts. Pedagogicseszka Zbornik, 1908.

966. Schmidt, A. See Logarithms. 
967. Schülke, A. Das Imaginäre im Unterricht. Schriften der phys.-ökonom. Gesellschaft in Königsberg i. Pr., 50 (1909).

968. — Über die Einführung negativer Zahlen. Zschr. math. natw. Unt., 37 (1906): 102 .

969. Seidler, H. Der casus irreducibilis für Mittelschulen. Zschr. für das Realschulw., 31 (1906): 76.

970. Short, R. L. Note on the use of fractional exponents. School Science and Mathematics, 8 (1908): 152.

971. Slaught, H. E. Aims in teaching algebra. School Science and Mathematics, 6 (1906): 105.

972. —_ What should be emphasized and what omitted in the high-school course in algebra. School Review, 16 (1908): 503.

973. Smith, D. E. Factoring of the type $p x^{2}+q x+r$. School Science and Mathematics, 7 (1907): 529.

974. - The nature of applied problems in algebra. School Science and Mathematics, 6 (1906): 482.

975. Smith, E. R. A shortened form of synthetic division and some of its applications. Mathematics Teacher, 3 (1910-11): 18.

976. Sommerville, F. H. Teaching of elementary algebra. Education, 24 (1908-09).

977. Stone, J. C. See McKinney, T. K.

978. Taylor, A. R. Suggestions for modifications in the order and time of presenting algebra. School Review, 13 (1905): 321.

979. Taylor, J. M. Equations. Mathematics Teacher, 2 (1909-10): 135.

980. Terry, H. S. Algebra in the high school. School Science and Mathematics, 10 (1910).

981. Thijn, A. Het onderwijs in der erste beginselen der algebra. Wiskundig Tijdschrift, 2 (1905).

982. Toan, B. E. Factoring of the type $p x^{2}-q x+r$. School Science and Mathematics, 7 (1907): 291.

983. Tresse, A. A propos des principes de l'homogénéité. Rev. de l'Ens. des Sciences, 2 (1908).

984. Tripp, M. O. Notes on the teaching of algebra in an Oberrealschule. Mathematics Teacher, 4 (1911-12): 35.

985. Valiron, G. See Arithmetic.

986. Weist, H. Bedeutung und Behandlung der Gleichungen im Unterricht. Progr. Städt. Realgymn. Görlitz, 1908.

\section{THE TEACHING OF GEOMETRY.}

987. Adler, A. Theorie der geometrischen Konstruktionen. Leipzig, Sammlung Schubert, Band 52.

988. Afnassjewa-Ehrenfest, T. Intuition und Logik im Mittelschulunterricht der Geometrie. (Russian.) Pädagogisches Museum der Kriegs Schulen, 1910.

989. Andrade, J. L'enseignement de la géométrie et les géométries non-Euclidiennes. L'Ens. Math., 2 (1900).

990. —_ Une leçon sur la géométrie de l'ajustage. L'Ens. Math., 8 (1906).

991. Appell, P. Notion de l'infini en géométrie élémentaire (à propos d'un article par M. Ripert). L'Ens. Math., 2 (1900): 127.

992. Arndt. See Erdmann.

993. Baker, A. L. Automatic diagrams in geometry. School Review, 10 (1902): 486.

$47398^{\circ}-12-4$ 
994. Baker, A. L. Limits in geometric forms. School Science and Mathematics, 5 (1905): 562 .

995. — Logical geometries. School Science and Mathematics, 6 (1906): 41.

996. The normal order of propositions in geometry; opening. School Science and Mathematics, 7 (1907): 94.

997. — Purposive geometry in the classroom. School Science and Mathematics, 6 (1906): 511 .

998. — The syllabus in geometry. School Science and Mathematics, 10 (1910).

999. Balser, L. Über die Verwendung der Parallelprojection im geometrischen Unterricht. Progr. Oberrealschule Darmstadt, 1910.

1000. Baudeuf, H. Contre la géométrie expérimentale. Rev. de l'Ens. des Sciences, 4 (1910).

1001. Beardsley, R. S. The teaching of geometry in its relation to the present educational trend. Discussion. National Education Association of the United States. Journal of proceedings and addresses, 1908. p. 638.

1002. Beman, W. W. Geometric constructions. School Science and Mathematics, $10(1910)$.

1003. Betz, W. Intuition and logic in geometry. Mathematics Teacher, 2 (190910): 3 .

1004. - Report of the national committee on the geometry syllabus and its practical pedagogical implications. School Science and Mathematics, 10 (1910): 684. National Education Association of the United States. Journal of proceedings and addresses, 1910. p. 511.

1005. - The teaching of geometry in its relation to the present educational trend. School Science and Mathematics, 8 (1908): 625; National Education Association of the United States. Journal of proceedings and addresses, 1908. p. 634 .

1006. Beuriger, J. Zur Lösung geometrischer Aufgaben. Zschr. math. natw. Unt., 38 (1907).

1007. Blum, R. Der Geometrieunterricht. Korrespondenzblatt, 17 (1910): 384.

1008. Bond, W. E. The aims in teaching geometry. Mathematics Teacher, 1 (1908): 17.

1009. Borel, E. À propos de l'enseignement de la géométrie élémentaire. Revue Scientifique, 8 (1907).

1010. Borgmeyer, J. Methodische Zusammenstellung des planimetrischen Lernstoffs für die mittleren Klassen. Progr. Kgl. Gymn. Meppen, 1907.

1011. Bourlet, C. Note sur la géométrie de M. Méray. Assoc. Franç. de Grenoble, 33 .

1012. _ La notion de groupe et la théorie des parallèles. L'Ens. Math., 9 (1907).

1013. — Thèse sur l'enseignement de géométrie. Bulletin de la Société Française de Philosophie, 7 (1907).

1014. Bozóky, E. A geométriai kutatás modzzereiröl. Über die Methoden der geometrischen Forschung. Középisk. Math. Lapok, 17 (1909).

1015. Brocke, E. Über die Benutzung symmetrischer Beziehungen im geometrischen Unterricht. Jahresber. Realschule Münster, Elsass, 1907.

1016. Buffa, P. A proposito di una proposta per l'insegnamento della geometria. nelle scuole medie inferiore. Boll. Mat., 3 (1904).

1017. Carson, G. St. I. The educational value of geometry. Reports of the Britis: Subcommission, 15.

In part a reprint from The functions of geometry as a subject of education. Tonbridge, 1910. See also On some unrealized possibilities in mathematical education. Mathematical Gazette, March, 1912. 
1018. Chase, H. J. How geometry should be learned. School Science and Mathematics, 8 (1908): 399.

1019. — Experimental geometry. School Science and Mathematics, 8 (1908): 577.

1020. Chatelet, A. Les principes de la géométrie; leur portée pour l'enseignement élémentaire. Rev. de l'Ens. des Sciences, 3 (1909).

1021. Chénard, H. Fusion de la géométrie plane et de la géométrie de l'espace. Rev. de l'Ens. des Sciences, 1 (1907).

1022. Chevalier, —. Quelques remarques sur l'enseignement de la géométrie par la méthode de M. Méray à l'école normale d'instituteurs. Assoc. Franç. de Grenoble, 33.

1023. Chiari, A. See Arithmetic.

1024. Child, J. M. The need of a sequence in geometry. Mathematical Gazette, 4 (1907).

1025. Ciamberlini, C. See Arithmetic.

1026. Cikot, C. A. Jets over vereenvoudiging van het elementair onderwijs in meetkunde. Wiskundig Tijdschrift, 2 (1905-06).

1027. Clairaut, A. C. Préface aux "Éléments de Géométrie." Printed in Rev. de l'Ens. des Sciences, 1 (1907).

1028. Combebiac, C. Sur le choix des principes dans l'enseignement de géométrie. Assoc. Franç. de Grenoble, 33.

1029. Davis, E. W. See Rouse, E. L., under Algebra.

1030. Dewey, J. The psychological and the logical in teaching geometry. Educational Review, 25 (1903): 287.

1031. Diekmann, J. Bewegung und Umformung. (Eine Skizze entwickelnder Geometrie für das Gebiet der Kreiskonstruktionen.) Zschr. math. natw. Unt., 35 (1904): 97.

1032. Dietrich, M. Der goldene Schnitt in der Schule. Blätter für das Gymn. Schulw., 38 (1902): 605.

1033. Dietsch, C. and Sievert, H. Aus dem Geometrie-Unterricht. Blätter für das Gymn. Schulw., 38 (1902): 605.

1034. Doerge, O. Über einige Beziehungen zwischen Geometrie und Arithmetik. Unterrichtsblätter, 16 (1910).

1035. Dressler, H. Abweichende Benennungen in Planimetrie und Stereometrie. Zschr. math. natw. Unt., 39 (1908): 593.

1036. Ducrue, J. Über den propädeutischen Geometrieunterricht in der vierten Gymnasialklasse. Unterrichtsblätter, 12 (1906).

1037. Durand, A. Sur le déplacement d'une figure dans le plan ou dans l'espace. Rev. de l'Ens. des Sciences, 2 (1908).

1038. Durrell, F. The locus problem in geometry with some discussion of the utilities in geometric study. School Science and Mathematics, 11 (1911).

1039. - Original demonstrations in geometry. Purpose, nature, and method of presentation. National Education Association of the United States. Journal of proceedings and addresses, 1907, p. 671; School Science and Mathematics, 7 (1907): 717.

1040. _ Teaching classes in geometry to solve original exercises. Mathematics Teacher, 1 (1908-09): 123.

1041. Ehrenfest, T. See Afnassjewa.

1042. Enquête sur l'enseignement de la géométrie. Rev. de l'Ens. des Sciences, 1 (1907).

1043. Erdmann and Arndt. Einführung in die Stereometrie als Pensum des I. Vierteljahres der I. Klasse. Progr. IV. Realschule Berlin, 1909. 
1044. Evans, G. W. A geometry pupil's brilliant work. School Science and Mathematics, 6 (1906): 595 .

1045. - Measurement theorems in geometry. School Review, 13 (1905): 307.

1046. Fabinger, F. The fundamental principles of the Euclidean geometry. (Bohemian.) Progr. Smichów (1902-03).

1047. Faragó, A. Über eine Induktion in der elementaren Geometrie. Zschr. für das Realschulw., 31 (1906): 213.

1048. Fehr, H. Vues stéreoscopiques pour l'enseignement de la géométrie. L'Ens. Math., 9 (1907).

1049. Ficquet, M. La géométrie dans les classes primaires. Rev. de l'Ens. des Sciences, 2 (1908).

1050. Finsterbusch, J. Geometrische Integrationen. Zwei Abhandlungen über neue Methoden zur Inhaltsbestimmung in der elementaren Geometrie. 32. Jahresbericht des Vereins für Naturkunde in Zwickau i. S., Leipzig, 1908.

1051. Fochtmann, —. Geometrische Örter. Lehrp. und Lehrg., 25 (1909): 415.

1052. Francis, W. A. Some changes in teaching geometry. School Science and Mathematics, 10 (1910).

1053. Frischauf, J. Über die Aufnahme der absoluten Geometrie in den höheren Unterricht. Zschr. math. natw. Unt., 33 (1902).

1054. G., C. Report on the teaching of geometry. Nature, 66 (1902): 201.

1055. Gaffney, M. A. The teaching of geometry, a way out. School Science and Mathematics, 8 (1908): 422.

1056. Gerlach, A. Das Masswerk im geometrischen Unterricht. Zschr. math. natw. Unt., 39 (1908): 341.

1057. Gille, A. Entspricht der geometrische Anfangsunterricht nach Euklid den Forderungen einer psychologischen Didaktik? Lehrp. und Lehrg., 20 (1904).

1058. Godfrey, C. The Board of Education circular on the teaching of geometry. Mathematical Gazette, 5 (1909-10): 195.

1059. - Is there need of a recognized sequence in geometry? Mathematical Gazette, 4 (1907).

1060. Greenwcod, G. W. Geometrical incongruities. School Science and Mathematics, 8 (1908): 250 .

1061. — Geometry report. School Science and Mathematics, 9 (1909).

1062. Limits in elementary mathematics. School Science and Mathematics, 8 (1908).

1063. - New terms in geometry. School Science and Mathematics, 8 (1908): 758.

1064. — Notes on geometry. School Science and Mathematics, 5 (1905): 369.

1065. - Trouble in solid geometry. School Science and Mathematics, 7 (1907): 102.

1066. Grossmann, M. Über die Aufgaben der Geometrie. Schweiz. Päd. Zschr., 1909.

1067. Haacke, F. Die Konstruktionsaufgabe im gymnasialen Unterricht. Lehrp. und Lehrg., 24 (1908): 405.

1068. Haas, M. Development of certain geometric propositions mechanically. (Bohemian.) Progr. Troppau, (1906-07).

1069. Haentzschel, E. Zur Genauigkeit geometrischer Konstruktionen. Unterrichtsblätter, 12 (1906); French summary in L'Ens. Math., 9 (1907): 45.

1070. Haffner, G. Anschauungsmittel zum propädeutischen Geometrieunterricht. Unterrichtsblätter, 16 (1910).

1071. Halsted, G. B. The teaching of geometry. Educational Review, 24 (1902): 456.

1072. Hamilton, J. G. The teaching of geometry. Mathematical Gazette, 4 (1907-08): 33 . 
1073. Hart, C. A. The teaching of geometry. School Science and Mathematics, 5 (1905): 649, 717 .

1074. Hart, H. F. Solid geometry. Mathematics Teacher, 3 (1910-11): 24 .

1075. - The way to begin solid geometry. Mathematics Teacher, 4 (1911-12): 54 .

1076. Hawkes, H. E. Some vital points in the teaching of geometry. School Science and Mathematics, 8 (1908): 222.

1077. Hedrick, E. R. The treatment of geometry for secondary instruction. National Education Association of the United States. Journal of proceedings and addresses, 1909. p. 515.

1078. Heinrich, M. Vereinfachter Lehrgang des Anfangsunterrichts in Planimetrie, analytischer Geometrie, und Trigonometrie. Progr. Luisen-Gymn. Berlin, 1911.

1079. - Vereinfachungen im planimetrischen Anfangsunterricht. Math. Natw. Blätter, 7 (1910).

1080. Hellweger, M. Die Huyghens'schen Methoden der Kreismessung, und Berechnung der Zahl $\pi$ auf 5 Dezimalen nach denselben. Progr. Brixen Privatgymn. (1906-07).

1081. Hill, M. J. M. Report of the Mathematical Association committee on the teaching of geometry and incommensurables. Mathematical Gazette, 2 (1901-04).

1082. Hioux, V. Parallélisme et translation rectiligne. L'Ens. Math., 9 (1907).

1083. Hogben, G. Notes on the teaching of elementary mathematics with special reference to geometry. Reports of the Australian Association, 10 (1905).

1084. Jackson, W. H. A simplification in elementary geometry. Mathematics Teacher, 3 (1910-11): 21.

1085. Janisch, W. Einige Aufgaben zur geometrischen Lehraufgabe in O. III. resp. U. II. Zschr. math. natw. Unt., 35 (1904): 43.

1086. —_ Eine neue Grundlegung der Geometrie. Zschr. math. natw. Unt., 41 (1910).

1087. Jones, F. T. See Algebra.

1088. Kelemen, I. A mértan tanitása. Der geometrische Unterricht. Polgárisk. Közl., 14 (1910).

1089. Kemlein, G. Der Fundamentalsatz der geometrischen Proportionen. Blätter für das Gymn. Schulw., 38 (1902): 678.

1090. Kleinpeter, H. Die Geometrie auf der Unterstufe. Zschr. für das Österr. Gymnasialw., 1904.

1091. Klug, R. Des kaiserlichen Mathematikers Johannes Kepler's Neujahrsgeschenk, oder über die Sechseckform des Schnees. Progr. Linz (1906-07).

1092. Koch, w. Zur Methode im geometrischen Unterricht. Progr. Sorau, 1906.

1093. Kopp, L. A geométria tananyagának beosztása nálunk és külföldön. Einteilung des geometrischen Lehrstoffes in Ungarn und im Ausland. See Beke-Mikola, Sammlung, under Secondary Schools.

1094. Kosmik, K. Das Dreieck, vornehmlich betrachtet in Bezug auf die ihm zukommenden besonderen Kreise und deren Radien. Progr. Mährisch Weisskirchen (1904-05).

1095. Kuhn, F. Beiträge zur Analysis des Beweises geometrischer Lehrsätze. Progr. Osnabrück, 1904.

1096. Kullrich, E. Bemerkungen über die Figuren des mathematischen Schulunterrichts. Zschr. math. natw. Unt., 38 (1907): 16.

1097. Laisant, C. A. Sur la méthode de M. Méray pour l'enseignement de la géométrie. Rev. de l'Ens. des Sciences, 1 (1907).

1098. Lasher, W. R. Some suggestions on decreasing the mortality in our geometry classes. Mathematics Teacher, 4 (1911-12): 26. 
1099. Lefèvre, A. L'enseignement de la géométrie dans les lycées de jeunes filles. Rev. de l'Ens. des Sciences, 2 (1908).

1100. Lennes, N. J. An attempt to adapt elementary geometry to the understanding and interests of young people. School Science and Mathematics, 10 (1910).

1101. - The treatment of limits in elementary geometry. School Science and Mathematics, 5 (1905): $743 ; 6$ (1906): 52 .

1102. Liard, L. L'enseignement de la géométrie dans les lycées et collèges de jeunes filles. Circulaire. Rev. de l'Ens. des Sciences, 1 (1907).

1103. Lietzmann, w. Eine französische Rundfrage über den geometrischen Unterricht. Päd. Arch., 15 (1908).

1104. — Die Grundlagen der Geometrie im Unterricht (mit besonderer Berücksichtigung der Schulen Italiens). Zschr. math. natw. Unt., 39 (1908): 177.

1105. — Der pythagorische Lehrsatz mit einem Ausblick auf das Fermatsche Problem. Mathemat. Bibliothek, 3. Leipzig, 1912.

1106. _ - Stoff und Methode des Raumlehrunterrichts auf Grund der Lehrbücher. Klein's Abhandlungen, V, 2.

1107. Liewald, K. Die Anschaulichkeit im geometrischen Anfangsunterricht. Zschr. math. natw. Unt., 40 (1909): 385.

1108. Löffler, E. Der Anfangsunterricht in der Geometrie. Korrespondenzblatt, 16 (1909).

1109. London. Board of Education. The teaching of geometry and graphic algebra in secondary schools. Circular no. 711, 1909.

A French translation by Bruce is contained in L'Ens. Math., 12 (1910): 238.

Opinions upon the Board of Education's circular published in School World (London), 11 (1909): 218.

1110. Lörcher, A. Zur Reform des Anfangsunterrichts in Geometrie. Korrespondenzblatt, 15 (1908).

1111. Lytle, E. B. Limits in elementary geometry. School Science and Mathematics, 10 (1910).

1112. Mack, K. Einige Bemerkungen zum Geometrieunterricht. Zschr. für•das Realschulw., 35 (1910).

1113. McPherron, J. M. Original demonstrations in geometry. Time of introduction and limitations in original demonstrations in geometry. National Education Association of the United States. Journal of proceedings and addresses, 1907. p. 671.

1114. Mannheim, A. Sur l'enseignement de la géométrie. L'Ens. Math., 7 (1905).

1115. Marotte, F. Les "Nouveaux Eléments de Géométrie" de M. Ch. Méray. Rev. de l'Ens. des Sciences, 1 (1907).

1116. Martin, P. Der Anschauungsunterricht in der Raumlehre nach Formengemeinschaften. Berlin.

1117. — Der gegenwärtige Stand der geometrischen Methodik, eine Rückantwort. Päd. Bausteine, Nr 20, Berlir.

1118. Méray, C. Justification des procédés et de l'ordonnance des "Nouveaux Eléments de Géométrie." L'Ens. Math., 6 (1904).

1119. — Les Nouveaux Eléments de Géométrie de M. Ch. Méray. Rev. de l'Ens. des Sciences, 2 (1908).

1120. — Opinions sur la méthode Méray pour l'enseignement de la géométrie. Rev. de l'Ens. des Sciences, 1 (1907).

See also Bourlet, Chevalier, Lalsant, Marotte, Perrin.
1121. Miller, G. A. On the development of elementary geometry in the nineteenth century. School Science and Mathematics, 7 (1907): 752.

1122. Minchin, G. M. The teaching of geometry. Nature, 80 (1909): 374 .

1123. Morawetz, J. Über die rechnende Geometrie an der Mittelschule. Progr. Staatsrealschule Salzburg (1906-07). 
1124. Moroff, A. Die Gleichheit in der Planimetrie. Blätter für das Gymn. Schulw., 37 (1901): 381.

1125. Muk, H. Auflösung eines schiefwinkeligen Dreiecks unter Benützung des Halbmessers des umgeschriebenen Kreises. (Bohemian.) Progr. Reichenau a. K. (1902-03).

1126. Müller, R. Über die Reformbestrebungen im geometrischen Unterricht an höheren Schulen, nebst einem Lehrplan für das mathematische Pensum in den drei Oberklassen der Oberrealschulen. Sitzungsber. der Berliner Math. Gesellschaft, 8 (1909); Lietzmann's Rubrik in Zschr. math. natw. Unt., 40 (1909).

1127. Nager, J. Einführung in die Elemente der geometrischen Analyse. Progr. Klosterneuburg (1908-09).

1128. Nath, M. Zur Methodik des geometrischen Anfangs-Unterrichts. Zschr. math. natw. Unt., 36 (1905): 1.

1129. Natucci, A. See Arithmetic.

1130. Nielsen, C. Neue Behandlung der Parallelenlehre. Unterrichtsblätter, 14 (1908).

1131. Noodt, G. Wie lassen sich die Meraner Vorschläge über die Reform des mathematischen Unterrichts für den geometrischen Unterricht an Lyceen verwerten? Frauenbildung, 5 (1906).

1132. Norrenberg, J. Die Methodik des geometrischen Anfangsunterrichts und die neuen preussischen Lehrpläne vom Jahre 1901. Zschr. für das Gymnasialw., 56 (1902): 230.

1133. Pannwitz, R. Psychologische Fehler im geometrischen Elementarunterricht. Zschr. für Phil. und Päd., 15 (1908).

1134. Patterson, C. A. A suggestion for review in geometry. School Science and Mathematics, 7 (1907): 704 .

1135. - Some thoughts on the teaching of geometry. School Science and Mathematics, 6 (1906): 756 .

1136. Peck, P. N. Special devices in teaching geometry. Mathematics Teacher, 3 (1910-11): 49.

1137. Perrin, E. La méthode de M. Méray pour l'enseignement de la géométrie. L'Ens. Math., 5 (1903): 441.

1138. Petit, G. L'enseignement de la géométrie aux débutants. Rev. de l'Ens. des Sciences, 2 (1908).

1139. Pfaff, H. Geometrische Örter als Übungstoff für die Prima. Zschr. math. natw. Unt., 37 (1906): 253, 321.

1140. Putney, E. N. Discussion on the teaching of geometry. Educational Review, 41 (1911): 200.

1141. Reisky, S. Zur Einführung in die geometrische Analysis. Progr. K. Kath. Gymn. Loetzschutz, 1906.

1142. Report of the Mathematical Association committee on geometry. Mathematical Gazette, 2 (1901-04): 168; Nature (1902).

1143. Richard, J. Sur l'enseignement de la géométrie; sur les mathématiques en philosophie. Rev. de l'Ens. des Sciences, 2 (1908).

1144. Richert, P. Umgestaltung der Parallelentheorie. Unterrichtsblätter, 14 (1908).

1145. Richter, O. Neue Elemente der Geometrie. Neue Jahrb., 8 (1905).

1146. Riechemeier, W. Das erste Jahr des planimetrischen Unterrichts am Gymnasium zu Gütersloh. Progr., 1906.

1147. Rigge, W.F. Polar triangles. School Science and Mathematics, 6 (1906): 663.

1148. Ripert, L. Sur la notion de l'infini en géométrie élémentaire. L'Ens. Math., 2 (1900): 370 . 
1149. Ripert, I. Sur l'utilité de la notion de l'infini dans l'enseignement de la géométrie élémentaire. L'Ens. Math., 2 (1900): 127.

1150. Roe, E. D. A generalized definition of limit. Mathematics Teacher, 3 (1910-11): 43 .

1151. - Some thoughts on space. Mathematics Teacher, 2 (1910-11): 31.

1152. Rorer, J. T. Interesting work of young geometers. Mathematics Teacher, 1 (1908-09): 150 .

1153. Rouse, E. R. See Algebra.

1154. Rousseau, H. La géométrie élémentaire basée sur le groupe de déplacements. L'Ens. Math., 11 (1909): 81.

1155. Row, T. Sundara. Geometric exercises in paper folding. Edited by Beman and Smith. Chicago, 1900.

1156. Russell, B. The teaching of Euclid. Mathematical Gazette, 2(1901-04): 165.

1157. Sainte Laguë, A. Un programme anglais de géométrie pratique. (With translation of the 1901 programme for the technical schools of the Board of Education.) Rev. de l'Ens. des Sciences, 4 (1910).

1158. _ Sur l'emploi de réseaux de parallèles équidistantes. Rev. de l'Ens. des Sciences, 3 (1909).

1159. Salomon, A. L'enseignement de la géométrie dans les lycées de jeunes filles. Rev. de l'Ens. des Sciences, 3 (1909).

1160. Sandars, A. Is the treatment of the theory of limits in our elementary geometries logical? School Science and Mathematics, 5 (1905): 396.

1161. Schacht, J. Die Ausbildung des räumlichen Anschauungsvermögens im mathematischen Unterrichte des Gymnasiums. Jahresber. Marien-Gymn. Posen, 1903.

1162. - Die methodische Verbindung der Planimetrie, Trigonometrie, und Stereometrie, zu einer einheitlichen Raumlehre. Progr. Marien-Gymn. Posen, 1908.

1163. — Ein neue Lehrgang für den Unterricht in der Raumlehre der höheren Lehranstalten. Jahresber. Marien-Gymn. Posen, 1906.

1164. Schaewen, P. von. Schüleraufgabe über rationelle Tetraedra. Zschr. math. natw. Unt., 35 (1904): 27.

1165. —— Stereometrische Analoga in planimetrischen Lehrsätzen. Zschr. für das Realschulw., 34 (1909).

1166. Schlesinger, J. Beitrag zur Lehre der Proportionalität der Linien; ein Beispiel von Grenzbetrachtung. Zschr. math. natw. Unt., 42 (1911).

1167. Schmehl, C. Über die methodische Behandlung einiger Fundamentalsätze der Geometrie vom Standpunkt der neueren Reformbestrebungen. Lehrp. und Lehrg. (1910).

1168. Schiilke, A. Welche Ziele hat der Unterricht in der Geometrie? Zschr. math. natw. Unt., 39 (1908).

1169. Schulte, T. H. Stereometrischer Unterricht in der Untersekunda der Realschulen. Monatschrift Höh. Schulw., 1907.

1170. Schultz, E. Über den einleitenden geometrischen Unterricht auf Quarta. Unterrichtsblätter, 11 (1905).

1171. Schultze, A. On the importance of numerical exercises in the teaching of geometry. School Science and Mathematics, 5 (1905): 247.

1172. Schulze, E. W. Grundzüge für eine pädagogische Reform und Abstufung des geometrischen Unterrichts in Quarta und Untertertia. Lehrp. und Lehrg., 1911.

1173. — Die Reformbestrebungen in der Methodik des geometrischen Anfangsunterrichts und die neuen preussischen Lehrpläne vom Jahre 1901. Zschr. für das Gymnasialw., 55 (1901). 
1174. Schuster, M. Über die constructive Behandlung der Stereometrie im Unterricht. Päd. Arch., 45 (1903).

1175. Schwatt, I. J. Some suggestions in the teaching of geometry. Mathematics Teacher, 2 (1909-10): 94 .

1176. Servit, F. Euclid's fundamental principles. (Bohemian.) Progr. Königliche Weinberge (1903-04); (1904-05); (1906-07).

1177. Sherk, W.H. Approximate values of $\pi$. Mathematics Teacher, 2 (1909-10): 84 .

1178. Shutts, G. C. Discussion of the report of the committee on geometry. School Science and Mathematics, 8 (1908): 199.

1179. Sievert, H. Über indirekte Beweise. Blätter für das Gymn. Schulw., 38 (1902): 386 .

See also Dietsch, C.

1180. Simon, M. Über die Entwickelung der Elementargeometrie im 19. Jahrhundert. Leipzig, 1906.

1181. Z Zur Frage der geometrischen Anschauungslehre. Südwestdeutsche Schulblätter, 24 (1907).

1182. Sisson, E. P. The aims of studying geometry and how to attain them. Mathematics Teacher, 1 (1908-09): 44.

1183. Skinner, E. B. Some hints on the use of limits in geometry. School Review, 17 (1909): 694 .

1184. Slaught, H. E. Geometrical syllabus. National committee of fifteen on geometry syllabus. Preliminary report. In National Education Association of the United States. Journal of proceedings and addresses, 1910. p. 511-15.

1185. - Provisional report. In National Education Association of the United States. Journal of proceedings and addresses, 1911. p. 607-29.

Reprinted in pamphlet form from School Science and Mathematics, April-June, 1911. [Chicago, 1911.] 77 p. $8^{\circ}$.

Bibliography: p. 2-4.

1186. Smith, D. E. The teaching of geometry. Boston, 1911.

1187. Smith, E. R. Elementary logic as a basis for plane geometry. Mathematics Teacher, 1 (1908-09): 6.

1188. _ The national geometry syllabus committee. Mathematics Teacher, 2 (1909-10): 164.

1189. — The syllabus method of teaching plane geometry. Mathematics Teacher, 1 (1908-09): 135.

1190. Soecknick, K. Zeichen, Benennungen, Definitionen in der Schulmathematik mit besonderer Berücksichtigung des Parallelenaxioms. Jahresber. des Friedrich-Kollegiums, Königsberg i. Pr., 1910.

1191. Stamper, A. W. The history of the teaching of elementary geometry with reference to present-day problems. New York, Teachers College, Columbia University, 1909.

1192. Stiessen, M. Beiträge zur Behandlung des Stereometrie-Unterrichts an der Realschule. Progr. Städt. Realschule, Königsberg i. Pr., 1901.

1193. Suppantschitsch, R. Die verschiedenen Arten des räumlichen Anschauungsvermögens. Zschr für das Österr. Gymnasialw., 60 (1909).

1194. Sutton, C. W. Some problems confronting teachers of geometry. School Science and Mathematics, 5 (1905): 139.

1195. Sykes, M. Some practical applications of elementary geometry. School Science and Mathematics, 6 (1906): 367.

1196. Szabó, P. A geométria tanítása az alsó osztälyokban. Der geometrische Unterricht in den unteren Klassen. See Beke-Mikola, Sammlung, under Secondary Schools.

1197. — A stereométria tanítása a VII. oszt.-ban. Der Unterricht der Stereometrie in der VII. Klasse. Progr. des Übungsgymn. Budapest (1904-05). 
1198. Szücs, A. A geométria tanitás ujabb módszereiröl. Über die neuen Methoden des geometrischen Unterrichts. Mag. Paed., 19 (1910).

1199. Tannery, J. L'enseignement de la géométrie. Revue Pédagogique, 46 (1903).

1200. Taylor, C. K. Teaching geometry: a criticism of the common method. Education, 30 (1910): 297.

1201. Thiede, J. Der Begriff der Inkommensurabilität im geometrischen Unterricht. Zschr. math. natw. Unt., 35 (1904): 198.

1202. Thieme, H. Die Parallelenlehre im Unterricht. Zschr. math. natw. Unt., 33 (1902): 549 .

1203. — Die Umgestaltung der Elementar-Geometrie. Progr. Posen, Nr. 175 (1900).

1204. Timerding, H. E. Für und wider die Dreieckskonstruktionen. Zschr. math. natw. Unt., 42 (1911).

1205. — Die Erziehung zur Anschauung. Leipzig, 1911.

1206. Treutlein, P. Anschauungsunterricht als Unterstufe eines zweistufigen Unterrichts in Geometrie. Leipzig, 1911.

1207. — Der geometrische Anschauungsunterricht. Leipzig, 1911.

1208. — Zur Frage der geometrischen Anschauungslehre. Südwestdeutsche Schulblätter, 24 (1907).

See also his Sammlung mathematischer Schulmodelle, Leipzig, 1912.

1209. Vareil, A. La géométrie par la méthode Méray. Rev. de l'Ens. des Sciences, 1 (1907).

1210. Veronese, G. Les postulats de la géométrie dans l'enseignement. Congr. Int. des Math. Compte Rendu du II. Paris, 1902.

1211. Volpi, R. Alcuni considerazioni sull'insegnamento della geometria sperimentale induttiva. Boll. Mat., 4 (1905).

1212. Walther, F. Die Neugestaltung des geometrischen Unterrichts. Unterrichtsblätter, 13 (1907).

1213. - Die Umgestaltung des geometrischen Unterrichts nach den Meraner Beschlüssen. Der Sämann, 4 (1908).

1214. Weist, H. Modelle zur räumlichen Veranschaulichung für die Hand der Schüler. Zschr. Lehrmittelwesen und päd. Literatur, 3 (1907).

1215. _ Zur stereometrischen Veranschaulichung. Zschr. math. natw. Unt., 36 (1905): 336 .

1216. Whitford, E. E. Geometry in the high school. School Science and Mathematics, 10 (1910).

1217. Witting, A. Einige Beweise elementarer planimetrischer Sätze. Zschr. math. natw. Unt., 42 (1911).

1218. Wolff, A. Über die Grundlagen unserer Schulgeometrie. Lehrp. und Lehrg., 24 (1908).

1219. Wooley, J. F. See Rouse, E. L., under Algebra.

1220. Zdelar, M. See General Topics.

1221. Zeissig, E. Die Raumphantasie im geometrischen Unterricht. Berlin, 1902.

\section{THE TEACHING OF TRIGONOMETRY.}

1222. Combet, E. Pour l'emploi des fonctions circulaires naturelles. With notes by C. Roubaudi. Rev. de l'Ens. des Sciences, 2 (1908).

1223. Delitala, G. La tetragonometria piana nelle scuole secondarie. Congr. Int. dei Mat. Atti del IV. Roma, 1909.

1224. Dintzl, E. Die erstèn Lehrstunden im Trigonometrieunterricht. Progr. Rainer-Realgymn., Wien, 1912.

1225. Fricke, F. Geometrische IIerleitung verschiedener trigonometrischer Formeln. Unterrichtsblätter, 14 (1908). 
1226. Glauer, R. Die trigonometrische Aufgabe in Untersekunda. Zschr. math. natw. Unt., 44 (1903): 397; Progr. Städt. Realschule Erfurt, 1902.

1227. Häbler, Th. Die Ausnahmslosigkeit beim Definiren trigonometrischer Funktionen. Zschr. math. natw. Unt., 37 (1906).

1228. Janisch, w. Die formelarme und logarithmenlose Methode der Auflösung trigonometrischer Aufgaben. Zschr. math. natw. Unt., 33 (1902): 551.

1229. Kreuschmer, R. Behandlung von trigonometrischen Vermessungsaufgaben im mathematischen Unterricht. Lehrp. und Lehrg., 23 (1907): 426.

1230. Lanner, A. Die tetragonometrischen Probleme in der Mittelschule. Zschr. math. natw. Unt., 40 (1909): 137.

1231. Lorey, w. Über die Genauigkeit bei angewandten Aufgaben aus der Trigonometrie. Zschr. math. natw. Unt., 41 (1910).

1232. Moritz, R. E. On the definition and scope of plane trigonometry. School Science and Mathematics, 8 (1908): 392.

1233. Moroff, A. Ist es notwendig oder sonst gerechtfertigt, den Sinus- und Kosinusbegriff nur für spitze Winkel aufzustellen? Blätter für das Gymn. Schulw., 38 (1902): 523.

1234. Schacht, J. See Geometry.

1235. Schlosser, A. Über ebene Trigonometrie und Lösung von Aufgaben. Progr. Tetschen a. E. (1907-08).

1236. Schmehl, Chr. Beiträge zur Methodik des Unterrichtes in der ebenen und sphärischen Trigonometrie. Progr. Grossherzogl. Oberrealschule Darmstadt, 1908.

1237. Schröder, J. Einige geometrische Herleitungen von goniometrischen Formeln. Zschr. math. natw. Unt., 39 (1908).

1238. Sommer, J. Einführung in die Trigonometrielehre. Progr. Prag Kleinseite (1904-05).

1239. Szabó, D. Az elsö trigonometriai ora. Die erste trigonometrische Lektion. Prog. des Uebungsgymnasium. Budapest, 1910.

1240. Szücs, E. Egyszerüsitések a trigonometria bizonyitásaiban. Vereinfachungen der trigonometrischen Beweise. Középisk. Math. Lapok, 17 (1910); Zschr. math. natw. Unt., 43 (1912).

1241. Wendler, A. Elementare Plan- und Kugelgeometrie im Zusammenhang mit der sphärischen Trigonometrie. Blätter für das Gymn. Schulw., 39 (1903): 72.

\section{THE TEACHING OF ANALYTIC GEOMETRY.}

1242. Baumgartner, A. A parabola módszeres tárgyalása. Methodische Behandlung der Parabel. Középisk. Math. Lapok., 17 (1910), 18 (1911).

1243. Carver, W. B. See Secondary Schools.

1244. Durell, C. V. A school course in advanced geometry. Reports of the British Subcommission, 16.

1245. Fiser, P. R. Rationale Zahlen in der analytischen Geometrie der Ebene. Progr. Braunau (1907-08).

1246. Heinrich, M. See Geometry.

1247. Kemlein, G. Zum Unterricht in der analytischen Geometrie an den humanistischen Gymnasien Bayerns. Progr. Hum. Gymn. Ludwigshafen a. Rh., 1905.

1248. Koeppner, K. Ausgewählte Kapitel aus der analytischen Geometrie in methodischer Behandlung. Lehrp. und Lehrg., 23 (1907): 194; 24 (1908): 298.

1249. Müller, H. Unvollkommene Darstellungen in Lehrbüchern der analytischen Geometrie und die mutmasslichen Ursachen derselben. Zschr. math. natw. Unt., 41 (1910): 529. 
1250. Rose, J. Sur l'enseignement de la géométrie analytique plane dans les écoles: secondaires. L'Ens. Math., 11 (1909): 190.

1251. Schoute, P. H. Une leçon de géométrie analytique. L'Ens. Math., 5 (1903): 106.

1252. Seidler, H. Die Verwendung des Krümmungsradius im Mittelschulunterrichte. Zschr. für das Realschulw., 30 (1905): 149.

1253. White, H. S. How should the college teach analytical geometry? Bulletin of the American Mathematical Society, 12 (1906): 493.

\section{THE TEACHING OF THE CALCULUS.}

1254. Beischer, K. Beispiele zur Differential- und Integral-Rechnung aus dem Gebiete der Physik. Progr. Oberrealschule Ravensburg, 1909; 1910.

1255. Beke, M. A differencziál- és integrál-számitás középiskolai anyagáról. Der Lehrstoff aus der Differential- und Integral-Rechnung an Mittelschulen. Zschr. math. natw. Unt., 41 (1910): 623. See Beke-Mikola, Sammlung, under Secondary Schools.

1256. Benesch, R. Die Differentiation der Exponential- und logarithmischen Funktion an der Mittelschule. Zschr. für das Realschulw, 32 (1907): 584.

1257. Berichte über die Ferienkurse in Göttingen. Especially the Zschr. math. natw. Unt., 39 (1908) by R. Schimmack, and 43 (1912) by A. Weinreich.

1258. Blutel, E. Sur une démonstration de la règle de l'Hospital. Rev. de l'Ens. des Sciences, 2 (1908).

1259. - Sur l'emploi des fonctions équivalentes. Rev. de l'Ens. des Sciences, 2 (1908).

1260. Bochow, K. Eine einfache und umfassende Methode zur Ableitung der Differentiation der Potenz und der Exponentialgrösse in Prima. Unterrichtsblätter, 17 (1911).

1261. Borel, E. See Molnár, E.

1262. Bryan, G. H. To reach the calculus as early as possible. Mathematical Gazette, 3 (1903).

1263. Buchrucker, B. Vortrag in der II. Versammlung der rheinländischen Professoren über die Einführung der Differentialrechnung. Bonn, 1908; Neue Jahrb. (1908).

1264. Bütler, K. Einführung in die Differential- und Integral-Rechnung für Schüler höherer Lehranstalten. Jahresber. der Kantonalen Industrieschule des Städt. Gymn. und der Secundarschule in Zug (1906-07).

1265. Capilleri, K. Die Einführung der Infinitesimalrechnung in der Realschule. Progr. Staatsrealschule Wien, 2 (1907-08).

1266. Carver. See Secondary Schools.

1267. Cikot, C. A. See Rapport van de commissie.

1268. Cornet, $\mathbf{L}$. Une démonstration simple de la règle de l'Hospital. Rev. de l'Ens. des Sciences, 1 (1907).

1269. Czuber, E. Die Frage der Einführung der Infinitesimalrechnung in den Mittelschulunterricht vom österreichischen Standpunkte. Jahresber. D. M. V., (1906): 116; Zschr. für das Realschulw., (1905): 641.

1270. Debat over de invoering van de infinitesmalrekening op de middelbare scholen. Wiskundig Tijdschrift, 2 (1905-06).

1271. Düsing, K. Lehrgang der Anfangsgründe der Differential- und IntegralRechnung an den höheren Maschinenbauschulen. Technik und Schule, 1 (1907).

1272. Ebner, F. Zur Frage der Infinitesimalrechnung an höheren Maschinenbauschulen. Technik und Schule, 1 (1907).

1273. — and Schülke, A. Infinitesimalrechnung im Unterricht. Unterrichtsblätter, 10 (1904). 
1274. Frank, H. Die Infinitesimalrechnung auf höheren Lehranstalten. Monatschrift für höhere Schulen, 2 (1903); 4 (1905).

1275. Franz, J. Zur Frage des Unterrichtes in der Infinitesimalrechnung an den höheren Lehranstalten. Unterrichtsblätter, 10 (1904).

1276. Frenzel, C. Die Fundamente für eine elementare Einleitung in die Differential- und Integral-Rechnung. Festschrift Gymn. Lauenburg i. Pr., II., Leipzig, 1910.

1277. Graves, G. H. Development of the fundamental ideas of differential calculus. Mathematics Teacher, 3 (1910-11): 82.

1278. Haacke, F. Die Maximalaufgabe als Einleitung in die Differentialrechnung. Zschr. math. natw. Unt., 38 (1907): 473.

1279. — Die Körperberechnung als Einleitung in die Integralrechnung. Zschr. math. natw. Unt., 40 (1909): 307.

1280. Habán, M. A differencziál- és integrálszámitás elemei alkalmazásokkal. Elemente und Anwendungen der Differential- und Integral-Rechnung. Jahresber. des Staatl. Obergymn. Erzsébetváros, 1909.

1281. Heffter, L. Differencziál- és integrál számitás. Vortrag. Differential-und Integralrechnung. (Ungarische Übersetzung von E. Molnár.) Középisk. Math. Lapok, 15 (1907).

1282. Herting, G. Von Strecke, Quadrat und Wurzel zum bestimmten Integral. Progr. Gymn. St. Anna Augsburg, 1910.

1283. Hočevar, F. Sind die Elemente der Infinitesimalrechnung an den Mittelschulen einzuführen oder night? Jahresber. D. M. V., (1906): 262; Zschr. für das Realschulw., (1906): 257.

1284. Holzmüller, G. Ist es möglich und wünschenswert die Differential- und Integralrechnung in den Lehrplan der höheren Schulen aufzunehmen? Monatschrift für höhere Schulen, 2 (1903).

1285. — Karl Schellbach und seine Stellung zur Frage der Differential- und Integral-Rechnung auf höheren Schulen. Unterrichtsblätter, 12 (1906).

1286. Hoppe, E. Die Elemente der Differential- und Integral-Rechnung im Lehrplan des humanistischen Gymnasiums. Progr. Wilhelm Gymn., Hamburg, 1910.

1287. Hubbes, H. Systematischer Lehrgang der Arithmetik und Algebra mit Berücksichtigung der Elemente der Differential- und Integral-Rechnung. Progr. Gymn. Brassó, (1910-11).

1288. Kántor, N. A differenciál- és integrálszámitás elemei. Elemente der Differential- und Integral-Rechnung. Progr. der Staatl. Oberrealschule in Eger, 1908.

1289. Kewitsch, G. Höhere Analysis in der Schule. Unterrichtsblätter, 11 (1905).

1290. Kubassa, C. Aufgaben für den Unterricht in Differenziren an Realschulen. Progr. Staatsoberrealschule Troppau, 1911.

1291. Laisant, C. A. L'enseignement du calcul. Paris, 1910.

1292. Lémeray, E. M. Sur l'enseignement élémentaire des fonctions elliptiques. L'Ens. Math., 5 (1903): 92.

1293. Lorey, w. Die Ableitung der Funktion $\mathrm{y}=\mathrm{x}^{\mathrm{m}}$. Zschr. math. natw. Unt., 40 (1909).

1294. — Die Behandlung des Taylor'schen Satzes in der Schule. Unterrichtsblätter, 17 (1911).

1295. — Über die Maclaurin'sche und Taylor'sche Entwickelung einer Funktion. Zschr. math. natw. Unt., 39 (1908).

1296. — Zur Differentialrechnung auf der Schule. Blätter höh. Schulw., 25 (1908). 
1297. Luckhaub, Gy. A függvénytan, a diff. és int. számítás bevezetése a középisko_ lába. Die Einführung der Funktionenlehre, der Differential- und IntegralRechnung in die höhere Schule. Vortrag. O. K. T. K., 40 (1907).

1298. Milarch, E. Der Differentialquotient im mathematischen Schulunterricht. Jahresber. Städt. Gymn. und Realgymn. Bonn, 1907.

1299. _ Eine elementare Verwertung der Differentialquotienten für die Berechnung von $\pi$. Zschr. math. natw. Unt., 38 (1907); 524.

1300. Molnár, E. A differencziálhányados elemi tárgyalása. Elementare Behandlung des Differentialquotienten; nach der Algebra von E. Borel, Középisk. Math. Lapok, 15 (1908).

1301. Nagele, L. Über die Einführung der Grundbegriffe der Infinitesimalrechnung in den Mittelschulunterricht. Wien, Plan der Staatsrealschule (1905-06).

1302. Newbold, W. See Secondary Schools.

1303. Osgood, W. F. The calculus in our colleges and technical schools. Bulletin of the American Mathematical Society, 13 (1907).

1304. — The teaching of calculus. Bulletin of the American Mathematical Society, 14 (1908): 165.

1305. Otta, M. The introduction of the calculus in the middle schools. (Bohemian.) Progr. Königliche Weinberge (1907-08).

1306. Ramaswami, A. The A B C of the higher analysis. Mathematical Gazette, 4 (1907).

1307. Rapport van de commissie belast met het onderzoek naar de wenschelijkheid en mogelijkheid van de invoering an de hoogere analyse of de middelbare school. Weekblad voor Gymn. en Midd. Ond. 3 (1907).

1308. Rátz, L. A függvények és az infinitesimál-számitas elemeinek tanitása középiskoláinkban. Unterricht der Elemente der Fuktionenlehre und des Infinitesimalkalküls an unseren höheren Schulen. See Beke-Mikola, Sammlung, under Secondary Schools.

1309. Report of discussions on elementary analysis at the Leicester meeting of the British Association. Mathematical Gazette, 4 (1907).

1310. Schiede, J. Die Begriffe der Funktion und des Differentialquotienten in Gymnasialprima. Jahresber. Gymn. Köslin, (1909).

1311. Schimmack, R. Eine specielle Frage der Schul-Infinitesimalrechnung. Unterrichtsblätter, 14 (1908).

1312. Schmall, C. N. High school course in the differential calculus. New York, 1907.

1313. Schmidt, J. Der Infinitesimalkalkül. Progr. Staatsrealschule, Wien, 9. (1908-09).

1314. - Zur Methodik der Infinitesimalrechnung. Zschr. math. natw. Unt., 41 (1910): 81 .

1315. Schöngut, L. Praktische Erfahrungen bei der Einführung der Infinitesimalrechnung im mathematischen Unterrichte der Mittelschule. Österr. Mittelschule, (1908): 459.

1316. Schrautzer, K. Zur Einführung der Unendlichkeitsrechnung in der Mittelschule. Progr. Staatsoberrealschule, Laibach, (1906-07).

1317. Schröter, K. Die bekannteren allgemeinen Methoden zur elementaren Bestimmung der Maximi und Minimi von Functionen mit einer veränderlichen Grösse. Jahrb. des Pädagogiums zum Kloster U. L. Frau, Magdeburg, 1906.

1318. Schülke, A. Differential- und Integralrechnung im Unterricht. Beilage zum Jahresber. der Kgl. Oberrealschule auf der Burg zu Königsberg i. Pr., 1907; Schriften der phys. ökonom. Gesellschaft in Königsberg, 47.

1319. — Differentielle et dérivée. L'Ens. Math., 13 (1911). 
1320. Schülke, A. Infinitesimalrechnung im Unterricht. Vortrag. Zschr. math. natw. Unt., 42 (1911).

See also Ebner, F.

1321. Schultze, E. Die Integralrechnung am Gymnasium. Unterrichtsblätter, 18 (1911).

1322. Schumann, E. Die höhere Mathematik in den württembergischen Oberrealschulen. Zschr. math. natw. Unt., 33 (1902): 441.

1323. Steckelberg, H. Die Elemente der Differential- und Integral-Rechnung. Wissenschaitliche Beilage zum Jahresber. des Realgymn. zu Witten, 1906.

1324. Tešar, L. Zur Frage der Behandlung der Infinitesimalrechnung im Mittelschulunterricht. Zschr. für das Realschulw., (1905): 276.

1325. Thaer, A. Differentialrechnung auf Realanstalten. Monatschrift für höhere Schulen, 3 (1904).

1326. — Zur Einführung in die Infinitesimalrechnung. Unterrichtsblätter, 19 (1910).

1327. Thiede, J.' Über die propädeutische Behandlung der Begriffe der Funktion und des Differentialquotienten in der Gymnasialprima. Zschr. math. natw. Unt., 42 (1911).

1328. Thieme, H. Zur Infinitesimalrechnung an Realanstalten. Zschr. math. natw. Unt., 38 (1907).

1329. Timerding, H. E. Die Infinitesimalrechnung auf der Schule. Vortrag. Sitzungsber. der Berliner Math. Gesellschaft, 10 (1911).

1330. Tuckey, C. O. The teaching of the elements of analysis. Report of British Association, 1907.

1331. Urban, A. Die Verwertung von $e$ im mathematischen Unterrichte. Zschr. math. natw. Unt., 39 (1908): 144.

1332. Weinmeister, P. Unendlichkeitsrechnung in der Schule. Zschr. math. natw. Unt., 38 (1907): 1.

1333. Well, G. J. van de. Die behandeling van het differentiaal begrip. Wiskundig Tijdschrift, 4 (1907).

1334. Wendler, A. Einführung in die Differential- und Integral- Rechnung auf Grund von Mittelwertsätzen. Unterrichtsblätter, 17 (1911).

1335. Werkmeister, P. Herleitung des Taylor'schen Satzes mit Hilfe der Figur. Zschr. math. natw. Unt., 40 (1909).

1336. Zahradniček, K. Eine neue Ableitung des Taylor'schen Theorems. Zschr. math. natw. Unt., 41 (1910).

1337. — Über die Frage der Verwendung der Infinitesimalrechnung beim Unterricht in der Mathematik und Physik an den österreichischen Mittelschulen. Österr. Mittelschule, 19 (1905): 36.

1338. _ Zur Frage der Einführung der Infinitesimalrechnung an den österreichischen Mittelschulen. Progr. Staatsrealschule, Wien, 2 (1904-05).

1339. _ Zur Frage der Infinitesimalrechnung an den österreichischen Mittelschulen. Vortrag, gehalten am IX. deutsch-österreichischen Mittelschultag, (1906). Österr. Mittelschule (1906): 189.

1340. Zimmermann, F. Zur Einführung der Infinitesimalrechnung. Progr. Landes Realschule und Obergymnasiums Horn, 1909. 


\section{THE TEACHING OF DESCRIPTIVE GEOMETRY AND GEOMETRICAL DRAWING.}

1341. Ács, I. A középiskolai rajztanításról. Über den Zeichenunterricht der höheren Schule. O. K. T. K., 41 (1908).

1342. Adler, A. Der Unterricht in der darstellenden Geometrie an den Realschulen und Realgymnasien. Berichte über den mathematischen Unterricht in Österreich, 9.

1343. Beyel, Chr. Die Bezeichnung in der darstellenden Geometrie. Zschr. math. natw. Unt., 34 (1903): 542.

1344. —_ L'enseignement de la géométrie descriptive dans les écoles moyennes. L'Ens. Math., 3 (1901): 431.

1345. Bleucke, Fr. Die Verbindung des Linearzeichnens mit dem stereometrischen Unterricht auf Untersekunda. Jahresber. Oberrealschule Essen, 1901.

1346. Bodenstedt, H. Geometrographie. Zschr. math. natw. Unt., 34 (1903): 32.

1347. — Ein Vortrag über Geometrographie. Zschr. math. natw. Unt., 35 (1904): 299.

1348. Bognár, P. C. A rajzoló géométria tanitásáról a gimnáziumban. Über den Unterricht der zeichnenden Geometrie am Gymnasium. Magyar Középiskola, 3 (1910).

1349. Bogner, - Wie leite ich die Hauptgesetze des perspectivischen Freihandzeichnens empirisch ab, und wie wende ich sie an? Bay. Zschr. für Realschulw., 17 (1909).

1350. Crelier, L. Le dessin de projection dans l'enseignement secondaire. L'Ens. Math., 6 (1904): 301.

1351. Diskussion über die Gestaltung des Unterrichts in der darstellenden Geometrie auf der X. Hauptversammlung des Vereins zur Förderung des Unterrichts in der Mathematik und der Naturwissenschaften zu Giessen. Mit Nachschrift von K. Hammdorf. Unterrichtsblätter, 7 (1901).

1352. Ducrue, J. Das stereometrische Zeichnen in der 8. Gymnasialklasse. Blätter für das Gymn. Schulw., 37 (1901): 401.

1353. Flegel, W. Der Zweck des Zeichenunterrichts an deutschen Schulen. Progr. Gymn. Recklingshausen, 1905.

1354. Gaubatz, M. Bericht über die Geometrie und darstellenden Geometrie im Wiener Verein, "die Realschule." Österr. Mittelschule, 22 (1909); 23 (1910).

1355. — Über Modelle und ihre Anwendung beim Unterricht in der darstellenden Geometrie. Z Zschr. Lehrmittelwesen und päd. Literatur, 6 (1911).

1356. Gercken, W. Die darstellenden Geometrie auf dem Realgymnasium nach den neuen preussischen Lehrplänen. Moratschrift für höhere Schulen, 1'(1902).

1357. Grothmann, H. Das Linearzeichnen an den preussischen höheren Schulen nach den Bestimmungen vom 17. IX. 1908. Schauen und Schaffen, 36 (1909).

1358. Grunewald, W. Die Zukunft des Linearzeichnens an den preussischen Realanstalten. Deutsche Blätter für Zeichen und Kunstunterricht, 9 (1901).

1359. Haage, K. Beiträge zur Geometrographie. Zschr. math. natw. Unt., 40 (1909): 494.

1360. Hammdorf, K. See Diskussion, etc.

1361. Hohlstein, P. Der Unterricht im Linearzeichnen an den preussischen Realanstalten nach der Verfügung vom 17. IX. 1908. Monatschrift für höhere Schulen, 8 (1909).

1362. Kirchberger, R. Die darstellende Geometrie an unseren Realschulen seit deren Bestande. Zschr. für das Realschulw., 31 (1906): 705.

1363. Kronke, F. Das Linearzeichnen in der Realschule. Progr. Städt. Oberrealschule zu Gaudenz, 1901. 
1364. Kullrich, E. and Wägler, E. Das Linearzeichnen. Jahresber. Realgymn. mit Realschule Gera, 1909.

1365. Massau, J. Discours sur l'enseignement de la géométrie descriptive. Ghent. 1366. Meissner, O. Über das Linearzeichnen in Verbindung mit dem mathematischen Unterricht in der Untersekunda. Progr. Realschule Pillau, 1901.

1367. Müller, E. Anregungen zur Ausgestaltung des darstellendgeometrischen Unterrichtes an technischen Hochschulen und Universitäten. Jahresber. D. M. V., 19 (1910).

1368. — Der Unterricht in der darstellenden Geometrie an den technischen Hochschulen. Berichte über den mathematischen Unterricht in Österreich, 9.

1369. Pietzker, F. Bericht über die darstellende Geometrie im Lehrplan der höheren Schulen. Unterrichtsblätter, 6 (1900).

1370. Pillet, F. J. La technologie dessinée dite dessin géométrique ou graphique. Rev..de l'Ens. des Sciences, 2 (1908).

1371. Quossek, K. Das Linearzeichnen der Oberrealschule. Zschr. für lateinlose höhere Schulen, 16 (1905).

1372. — Linearzeichnen und darstellende Geometrie. Zschr. für lateinlose höhere Schulen, 18 (1907).

1373. Reinhardt, R. Die Bedeutung des in den Händen der Schüler befindlichen Zeichenmaterials für die Erfolge des Zeichenunterrichts. Progr. Realschule Oberstein-Idar, 1902.

1374. Renbach, W. On the importance of descriptive geometry in the programmes of general education. (Polish.) Spraw. dyr. szk. real, 1907.

1375. Richter, A. Die Übertragung des Unterrichtes im Linearzeichnen an die Mathematiklehrer. Zschr. math. natw. Unt., 33 (1902): 46.

1376. Richter, H. Das geometrische Zeichnen. Progr. I. Realschule Leipzig, 1901.

1377. Richter, M. Über die Einführung in die Stereometrie und in das stereometrische Zeichnen. Leipzig, 1910.

1378. Richter, O. Das geometrische Zeichnen in der Realschule. Beilage zum Jahresber. der I. Realschule zu Leipzig, 1901.

1379. Roubaudi, C. Géométrie et dessin géométrique. Rev. de l'Ens. des Sciences, 4 (1910).

1380. L Le programme de dessin graphique dans le premier cycle B. des lycées et collèges. Rev. de l'Ens. des Sciences, 1 (1907).

1381. Rühlmann, K. Zwei Wünsche zum Linearzeichnen. Zschr. math. natw. Unt., 34 (1903): 437.

1382. Schiffner, F. See Preparation of Teachers.

1383. Schilling, F. Über die Anwendungen der darstellenden Geometrie, insbesondere über die Photogrammetrie. See Klein-Riecke, Neue Beiträge, under Correlation.

Also printed separately, Leipzig, 1904.

1384. Schmehl, Chr. Neue Modelle für den Unterricht in der darstellenden Geometrie, Perspektive, und rechtwinkligen Axonometrie. Zschr. math. natw. Unt., 34 (1903): 245.

1385. Schotten, H. Das Zeichnen in seinen Beziehungen zum naturwissenschaftlichen und erdkundlichen Unterricht. Zschr. math. natw. Unt., 33 (1902): 520.

1386. Schuberth, A. Über den bildenden Wert und die Bedeutung der darstellenden Geometrie in den österreichischen Mittelschulen. Österr. Mittelschule, 23 (1909).

1387. Schütte, Fr. Anfangsgründe der darstellenden Geometrie für Gymnasien. Progr. des Realgymn. zu Düren. Leipzig, 1906.

$47398^{\circ}-12 \longrightarrow 5$ 
1388. Sellenthin, R. Lehrgang der Linearperspective für höhere Lehranstalten. Beilage des Jahresber. der Oberrealschule zu Elberfeld, 1903.

1389. - Methodischer Lehrgang des Linearzeichnens für höhere Lehranstalten. Beilage des Jahresber. der Oberrealschule zu Elberfeld, 1904.

1390. Tiedge, E. Die darstellende Geometrie in den höheren Schulen Preussens. Päd. Arch., 51 (1909).

1391. Twrdy, K. Ein Beitrag zum kristallographischen Unterricht in der VII. Klasse der österreichischen Realschulen. Zschr. für das Realschulw., 28 (1903): 65 .

1392. Verordnungnen für den Zeichenunterricht an preussischen Realanstalten. Unterrichtsblätter, 14 (1908).

1393. Wägler, E. See Kullrich, E.

1394. Weinbeer, E. W. Die statischen Konstruktionen im Unterricht des geometrischen Zeichnens. Monatschrift für höhere Schulen, 9 (1910).

1395. Weise, K. Zur gegenwärtigen Lage des Unterrichts in der zeichnenden Stereometrie. Zschr. für lateinlose höhere Schulen, 15 (1904).

1396. Zühlke, P. Bericht über die seit 1901 erschienenen Schulbücher der darstellenden Geometrie. Sitzungsber. der Berliner Math. Gesellschaft, 9 (1910).

1397. — Mathematiker und Zeichenlehrer im Linearzeichenunterricht der preussischen Realanstalten. Berichte und Mitteilungen, veranlasst durch die I. M. U. K., 4; Zschr. math. natw. Unt., 41 (1910).

1398. — Über den Unterricht in der darstellenden Geometrie. Vortrag. Zschr. math. natw. Unt., 42 (1911).

1399. __ Der Unterricht im Linearzeichnen und in der darstellenden Geometrie in den deutschen Realanstalten. Klein's Abhandlungen, III, 3.

Contains a good bibliography.

\section{THE VALUE AND THE TEACHING OF GRAPHS.}

1400. André, D. Résolution graphique de l'équation $x^{2}-p x+q=0$. Nouv. Annales de Math., $4^{\circ}$ série, 5 (1905).

1401. Ayné, P. Nouvelles applications des méthodes graphiques aux opérations financières. Revue Générale des Sciences, 15 (1904).

1402. Beman, W. W. Graphic railroad time-tables. School Science and Mathematics, 10 (1910).

1403. Bérard, R. Sur la construction graphique des racines d'une équation du second degré. Rev. de l'Ens. des Sciences, 2 (1908).

1404. Bettazzi, R. La représentation graphique des nombres. L'Ens. Math., 3 (1901).

1405. Betz, W. Graphic methods in elementary algebra. School Science and Mathematics, 6 (1906): 683.

1406. Blakslee, T. M. Graphical solution of quadratic with complex roots. School Science and Mathematics, 11 (1911).

1407. Board of Education. See Geometry.

1408. Boon, F. C. Graph tracing. School Science and Mathematics, 5 (1905): 398.

1409. Brand, E. Méthodes graphiques pour déterminer les racines réelles de l'équation $x^{2}-p x+q=0$. L'Ens. Math., 8 (1906).

1410. Büchel, W. Beitrag zur Anwendung graphischer Darstellung auf der Mittelschule. Zschr. math. natw. Unt., 39 (1908): 608.

1411. Cajori, F. Graphic railroad time-tables. School Science and Mathematics, $10(1910)$.

1412. Courcot, A. Résolution graphique de l'équation du troisième degré. Rev. de l'Ens. des Sciences, 2 (1908). 
1413. Darnell, A. A graphical solution of the quadratic equation. School Science and Mathematics, 11 (1911).

1414. Deny, L. Note sur la représentation géométrique des polynomes algébriques. Nouv. Annales de Math., $4^{\circ}$ série, 5 (1905).

1415. Dickson, I. E. Graphical methods in trigonometry. American Mathematical Monthly, 1905.

1416. Doležal, E. Graphische Darstellungen auf Grund der Flächengleichungen. Jahresber. für Berg- und Hüttenwesen, Leoben, 1907.

1417. Doolittle, F. W. See Epstein, S.

1418. Dougall, J. Graphical treatment of geometrical progression. Mathematical Notes, Edinburgh, 1 (1911).

1419. Ebner, F. Neue graphische Darstellungen. Zschr. gewerbl. Unt., 24 (1909).

1420. Engelhardt, P. Über die graphischen und näherungsweise numerischen Auflösungen von Gleichungen II. ụnd III. Grades mit einer Unbekannten an der Mittelschule. Progr. Würzburg, 1910.

1421. Epstein, S. and Doolittle, F. W. Graphical illustrations of convergence of series. School Science and Mathematics, 7 (1907): 770.

1422. Evans, G. W. Arithmetic and algebra-graphical representation. School Review, 11 (1903): 618.

1423. Gallander, 0 . Forberedende kurs i grafisk algebra. Stockholm.

1424. Glenn, O. E. The geometric representation of the roots of equations. American Mathematical Monthly, 14 (1907).

1425. Goldziher, K. A másodfokú egyenlet grafikai tárgyalása. Graphische Behandlung der Gleichung zweiten Grades. Középisk. Math. Lapok, 17 (1910); im Auszug, Unterrichtsblätter (1912): 1.

1426. — A mértani sor grafikai vizsgálata. Graphische Behandlung der geometrischen Reihe. Középisk. Math. Lapok, 17 (1910).

1427. — A számtani sor grafikai vizsgálata. Die graphische Behandlung der arithmetischen Reihe.. Középisk. Math. Lapok, 16 (1908).

1428. — Grafikai módszerek a számtani oktatásban. Graphische Methoden im Rechenunterricht. O. K. T. K., 41 (1908); Beke-Mikola, Sammlung (ungarische Ausgabe). See Secondary schools.

1429. —_ Les méthodes graphiques en mathématiques. Bibliographie. Rev.de l'Ens. des Sciences, 1 (1907).

1430. _ Über die Anwendung des graphischen Verfahrens im mathematischen Schulunterricht. Unterrichtsblätter, 15 (1909).

1431. Goy, P. de. Rapport sur le mémoire de l'abbé Thorelle intitulé: Solutions graphiques des problèmes d'arithmétique à l'aide du papier quadrillé. Metz, Mémoires de l'Académie, $3^{\mathrm{e}}$ série, 33 (1903).

1432. Grosse, W. Die graphische Behandlung der Gleichungen im Unterricht. Zschr. math. natw. Unt., 37 (1906): 267.

1433. Hayashi, T. Graphic solution of the cubic and quartic. (With notes by Vacca.) Nature, 63 (1900-01).

1434. Heck, R. C. H. Notes on the graphics of machine forces. New York, 1911.

1435. Kuhn, I. Graphische Darstellung und elementare Berechnung einiger Integrale. Zschr. für das Realschulw., 33 (1908).

1436. Lehrfeldt, R. A. Note on the graphical treatment of experimental curves. Philosophical Magazine, series VI, 1 (1901).

1437. Lennes, N. J. Graph in high-school mathematics. School Review, 14 (1906): 339.

1438. Lesser, O. Graphische Darstellungen im Mathematikunterricht der höheren Schulen. Leipzig, 1908. 
1439. Marotte, F. Résolution graphique de l'équation $a \sin x+b \cos x=c$. Rev. de l'Ens. des Sciences, 1 (1907).

1440. Mehmke, R. Graphisches Rechnen. Kap. III. des Encyklopädiereferates "Numerisches Rechnen." Encykl. der Math. Wissensch., I, 953.

1441. Mettler, H. Graphische Berechnungsmethoden im Dienste der Naturwissenschaften und Technik. Zürich, 1910.

1442. Milankowitsch, M. Eine graphische Darstellung der geometrischen Progression. Zschr. math. natw. Unt., 40 (1909).

1443. Milne, R. M. Graphic methods in an educational course on mechanics. Nature, 70 (1904): 5.

1444. Mittag, M. Zurgeometrischen Darstellung algebraischer Formeln. Jahresber. Hgl. Anh. Landessem., 19 Cöthen, O. 1901.

1445. Moore, E. H. Cross-section paper as a mathematical instrument. School Review, 14 (1906): 317; School Science and Mathematics, 6 (1906): : 431.

1446. Noaillon, P. Résolution graphique de l'équation du III ${ }^{\circ}$ degré. Mathesis, Paris, $3^{\mathrm{e}}$ série, 7 (1907).

1447. Nonne, Th. Zinseszinz und Rentenrechnung mit Hilfe graphischer Darstellung. Berlin, 1903.

1448. Noodt, G. Graphische Darstellung im Rechenunterricht an höheren Mädchenschulen. Frauenbildung, 7 (1908).

1449. Oberbach, J. Graphische Darstellung beim Unterricht in der Handelstechnik. Zschr. für das gesamte kaufmänn. Unterrichtsw., 9 (1907).

1450. Ocagne, M. d'. Calcul graphique et nomographie. Encyclopédie Scientifique, Paris, 1908.

1451. _ La méthode graphique en mathématique appliquée. Revue du Mois, 1 (1906).

1452. - La nomographie dans l'enseignement. L'Ens. Math., 2 (1900).

1453. Orbán, Gヌ. A mérátani sor grafikai vizsgálata. Graphische Behandlung der geometrischen Reihe. Középisk. Math. Lapok, 17 (1908).

1454. Pages, A. La notion de lieu géométrique acquise par la construction graphique. Rev. de l'Ens. des Sciences, 1 (1907).

1455. Péch, A. A grafikus módszer alkalmazásáról. Über die Anwendung des graphischen Verfahrens. See Beke-Mikola, Sammlung, under Secondary Schools.

1456. Poussin, R. Sur l'application des procédés graphiques aux calculs d'assurance. (Especially chap. I.) Bulletin Trimestriel des Actuaires Français, N. 58, 1904.

1457. Pratt, A. Calculation by geometry. Practical Teacher, 1903.

1458. Prieur, —. Les méthodes graphiques en mathématiques. Bibliographie. Rev. de l'Ens. des Sciences, 1 (1907).

1459. Richert, P. Die ganzen rationalen Funktionen der ersten drei Grade und ihre Kurven. Progr. 3. Realschule, Berlin, 1907.

1460. Rottsieper, W. Zur Konvergenz der geometrischen Reihe. Unterrichtsblätter, 16 (1910).

1461. Sainte Laguë, A. Note sur les usages du papier quadrillé. L'Ens. Math., 12 (1910): 5 .

1462. Salow, A. Über die Benutzung der graphischen Darstellung im mathematischen Unterricht der Mittelstufe. Päd. Arch., 60 (1911).

1463. Schlags, W. Geometrische Lösung algebraischer Gleichungen. Leuchtturm (Trier), 1910.

1464. - Graphische Lösung der Gleichung $\mathrm{x}^{2}+\mathrm{ax} \pm \mathrm{b}=0$. Unterrichtsblätter, 16 (1910).

1465. Schotten,H. Einige Beispiele zur graphischen Darstellung. Zschr. math. natw. Unt., 41 (1910): 467. 
1466. Schröder, J. Beiträge zur Anwendung des graphischen Verfahrens im mathematischen Unterricht. Beiträge zum Jahresber. Oberrealschule vor dem Holstentore, Hamburg, 1909.

1467. Sieber, A. Graphische Lösung höherer algebraischer Gleichungen. Schweizerische Bauzeitung, 32 (1901).

1468. Smith, A. W. What results are we getting from graphic algebra? Mathematics Teacher, 4 (1911-12): 21.

1469. Sós, E. A számtani sor összegezö tagjának kiszámitása. Graphische Berechnung der Summe einer arithmetischen Reihe. Középisk. Math. Lapok, 16 (1909).

1470. — Grafikai módszerek a kereskedelmi számtanban. Graphische Methoden in der kaufmännischen Arithmetik. Kereskedelmi Szakoktatás, 18 (1909-10).

1471. Stuyvaert, M. Conséquences diverses d'une formule algébrique; leurs interprétations géométriques. L'Ens. Math., 8 (1906).

1472. Szántó, H. Grafikus és mérömódszerek a számtani oktatásban. Graphische und messende Methoden im Rechenunterricht. Nemzeti Népoktatás, 1911.

1473. Szijártó, M. A grafikus módszer felhasználása az algebrai tanitás kezdetén. Die graphischen Methoden beim Beginn des algebraischen Unterrichts. Progr. des Budapester Mädchengymnasiums des Frauenbildungs-Vereines, 1905.

1474. Tanikiewits, V. A függvények ismertetése és ábrázolása a VII. osztályban. Behandlung und Darstellung der Funktionen in der VII. Klasse. Jahresber. der Staatl. Oberrealschule Székesfehérvár, 1909.

1475. Thomson, H. I. Graphical solution of cubic and quartic equations. Nature, 70 (1905).

1476. Torrès, L. Sur les rapports entre le calcul mécanique et le calcul graphique. Bull. Soc. Math., 29 (1901).

1477. Touton, F. C. Graph work in elementary algebra. School Science and Mathematics, 5 (1905): 557.

1478. Wallis, - Note on the graphic solution of simultaneous equations in three unknowns. Educational Times, 57 (1904).

1479. Weill, A. Über die graphische Bestimmung der Kreisfläche auf III. sup. der Gymnasien. Zschr. math. natw. Unt., 41 (1910).

1480. — Graphischer Aufgaben Sammlung. Gebweiler, 1911.

1481. Werkmeister, P. Beitrag zur graphischen Darstellung von Gleichungen der Form $a b-c=0 . \quad$ Zschr. für Math. und Phys., 57 (1909).

\section{THE TEACHING OF LOGARITHMS AND THE SLIDE RULE.}

1482. Arbes, J. Sollen vierstellige Logarithmen an den österreichischen Mittelschulen eingeführt werden? Zschr. für das Realschulw., 30 (1905): 207.

1483. — Über Erfindung, Gestaltung und Wertschätzung der Logarithmen. Progr. Smichów, (1906-07), (1907-08).

1484. _ Z Zur Einführung vierstelliger Logarithmentafeln in die Mittelschulen. Österr. Mittelschule (1904): 373.

1485. Aubert, J. La règle à calcul au baccalauréat. Rev. de 1'Ens. des Sciences, 1 (1907).

1486. Bauernberger, A. Eine methodische Behandlung der Rechengesetze für Potenzen, Wurzeln, und Logarithmen. Zschr. für das Österr. Gymnasialw., 62 (1911).

1487. Cahn, E. See Mineur, P.

1488. Dobbs, W. J. A practical introduction to indices and logarithms. School World, 1909. 
1489. Glauer, R. Aufgaben für das Rechnen mit vierstelligen Logarithmen. Zschr. math. natw. Unt., 33 (1902): 370.

1490. Hammer, E. Der logarithmetische Rechenschieber und sein Gebrauch. Stuttgart, 1908.

1491. Hinckley, F. C. and Ramsay, W. W. The slide rule. Boston, 1911.

1492. Jackson, C. S. The slide rule and its use in teaching logarithms. Mathematical Gazette, 2 (1903).

1493. Junge, G. Les logarithmes de base 1.01. Rev. de 1'Ens. des Sciences, 2 (1908).

1494. Kullrich, E. Bęmerkungen zur Rezeichnung der Logarithmisirung. Unterrichtsblätter, 14 (1908).

1495. Lissák, J. Handbuch des Rechenschiebers. (Hungarian.) Budapest, 1911.

1496. McLeed, C. Practical introduction of logarithms. School World, 3 (1909).

1497. Mayer, T. E. Das mechanische Rechnen und seine Hilfsmittel. Leipzig, 1908.

1498. Meyer, E. Die Lehre von den Logarithmen. Zschr. math. natw. Unt., 39 (1908).

1499. Milarch, E. Elementare Berechnung der Logarithmen. Zschr. math. natw. Unt., 37 (1906): 43.

1500. Mineur, P. Une exposition élémentaire de la théorie des logarithmes. (With notes by E. Cahn and A. Mauduit.) Rev. de l'Ens. des Sciences, 1 (1907)

1501. Moritz, R. E. On the computation of common logarithms. School Science and Mathematics, 9 (1909).

1502. Müller, C. H. Der logarithmetische Rechenstab und die Schule. Zschr. math. natw. Unt., 38 (1907): 526.

1503. Myers, G. W. Logarithms in the first year of the secondary school. School Science and Mathematics, 5 (1905): 709; 6 (1906): 99 .

1504. - The meaning and use of the three-place table. School Science and Mathematics, 6 (1906): 204.

1505. Ocagne, M. d'. Instruction sur l'usage de la règle à calcul. Paris, 1910 .

1506. Otti, - L. Les tables de logarithmes à quatre décimales et la division décimale de l'angle dans l'enseignement des écoles moyennes. Schweiz. Päd. Zschr., 1906.

1507. Quint, N. Elementare berekening van logarithmen. Wiskundig Tijdschrift, 2 (1906).

1508. Ramsay, W. W. See Hinckley, F. C.

1509. Richter, O. Dreistellige Logarithmen. Zschr. math. natw. Unt., 39 (1908): 373.

1510. Rozé, P. Théorie et usage de la règle. Paris, 1907.

1511. Schmidt, A. Beiträge zum mathematischen Unterricht. 1. Berechnung von Logarithmen. 2. Einführung der complexen Zahlen. Jahresber. Kgl. Prinz Heinrich Gymn., Berlin, (1905).

1512. Schubert, H. Elementare Berechnung der Logarithmen. Zschr. math. natw. Unt., 34 (1903): 497, 551; 35 (1904): 273; and Leipzig, 1903.

1513. Schülke, A. Über die Bezeichnung der Logarithmen. Unterrichtsblätter, 15 (1909).

1514. — Vierstellige Logarithmen. Blätter für das Gymn. Schulw., 39 (1903): 629.

1515. Schumacher, R. Ein Rechenschieber. Bay. Zschr. für Realschulw., 17 (1909).

1516. Spiess, J. Zur elementaren Berechnung der Briggsschen Logarithmen. Math. Natw. Blätter, 3 (1906).

1517. Suppantschitsch, R. Sur une introduction première des logarithmes. L'Ens. Math., 11 (1909): 374. 
1518. Tresse, A. Un mode d'exposition de la théorie des logarithmes. Rev. de l'Ens. des Sciences, 5 (1911).

1519. Vital, A. Über die Methode der Berechnung einer Logarithmen-Tafel auf elementarem Wege. Zschr. für das Realschulw., 25 (1900): 22.

1520. Weisner, —. Der Rechenstab im Unterricht an Maschinenbauschulen. Technik und Schule, 1 (1907).

1521. Winter, J. Táblázatok és számolóléc. Tabellen und Rechenschieber. See Beke-Mikola, Sammlung, under Secondary Schools.

\section{THE INTRODUCTION OF THE FUNCTION CONCEPT.}

1522. Albrich, K.,jr. Behandlung der Funktionen im Mittelschulunterricht. Progr. des evang. Gymn. A. B., Nagyszeben, 1906.

1523. Antal, M. A függvények változásának és ábrázolásának tanulmányozása. Studium der Veränderung und der Darstellung von Funktionen. Középisk. Math. Lapok, 16 (1909).

1524. Dintzl, E. Einführung in die Funktionenlehre. Jahresber. K. K. Erzherzog Rainer Gymn., Wien, 1908.

1525. Dressler, H. Die Bedeutung functioneller Beziehungen in der Geometrie. Lehrp. und Lehrg. (1911).

1526. Fehr, H. Der Funktionsbegriff im mathematischen Unterricht der Mittelschule. Vortrag und Discussion. Verhandlungen der Vereinigung der Mathematiklehrer an schweizerischen Mittelschulen (1905).

152i. — La notion de fonction dans l'enseignement mathématique des écoles moyennes. L'Ens. Math., 7 (1905): 177.

1528. Geissler, K. Die Funktion wissenschaftlich und im Unterricht. Zschr. für lateinlose höhere Schulen, 19 (1908).

1529. — Zur Frage der stetigen Funktion ohne Differentialquotienten. Unterrichtsblätter, 15 (1909).

1530. Goering, W. Eine Abiturientenprüfung im funktionalen Denken. Zschr. math. natw. Unt., 39 (1908); Verhandl. der Unterrichtskommission der G. D. N. u. Ä., 1908.

1531. Hack, - Beispiele aus der Elementarmathematik und verwandten Gebieten zur Einführung in den Funktionsbegriff. Zschr. math. natw. Unt., 41 (1910): 1.

1532. Josephson, 0 . On begreppet derivata och dess användning för studiet av enkla funktioner. Växjö, 1909.

1533. Lesser, O. Die Entwickelung des Funktionenbegriffs und die Pflege des funktionalen Denkens im mathematischen Unterricht unserer höheren Schulen. Festschrift der Klinger Oberrealschule, Frankfurt a. M., 1907.

1534. Maltson, R. Funktionsbegreppets införande i matematikundervisningen. Ped. Tidskr., 43 (1907).

1535. Mendelssohn, V. Die Erleicbterung des mathematischen Unterrichts durch Einführung des Funktionsbegriffs. Math. Natw. Blätter, 8 (1911).

1536. Quossek, K. Die Entwickelung des Funktionsbegriffs und die Pflege des funktionalen Denkens im Mathematikunterricht unserer höheren Schulen. Zschr. für lateinlose höhere Schulen, 18 (1907).

1537. Rohrauer, G. Das funktionale Denken im Mittelschulunterricht. Österr. Mittelschule, (1909).

1538. Schmehl, Chr. Die Entwickelung des Funktionsbegriffs im Mathematikkurs der höheren Schulen. Päd. Arch., 51 (1909).

1539. Schmidt, W. Wie gewinnen wir für die Behandlung des Funktionsbegriffs Platz im mathematischen Unterricht? Beilage zum Progr. N ${ }^{r} .595$ des Realgymn. in Düren (1906). 
1540. Schnee, W. Der Funktionsbegriff im mathematischen Unterricht des Gymnasiums. Math. Natw. Blätter, 7 (1910).

1541. Schreber, K. Das funktionale Denken im Physikunterricht. Zschr. phys. chem. Unt., 20 (1907).

1542. Schultz, E. Über das Funktionale in der Geometrie. Unterrichtsblätter, 15 (1909).

1543. Tanfi, I. Bevezetés a mennyiségtani függvényfogalomba. Einführung in den mathematischen Funktionsbegriff. Néptanitók Lapja, 41 és 42 (1908-9).

1544. Thiede, J. Die Begriffe der Funktion und des Differentialquotienten in der Gymnasialprima. Progr. Gymn. Köslin, 1909.

1545. Wendler, A. Funktion und Invariante im mathematischen Unterricht. Blätter für das Gymn. Schulw., 1906.

1546. — Über Funktionalgleichungen in der Elementarmathematik. Unterrichtsblätter, 14 (1908).

1547. — Weiteres über Funktionalgleichungen in der Elementarmathematik. Unterrichtsblätter, 15 (1909).

\section{THE QUESTION OF CORRELATION AND THE APPLI- CATIONS OF MATHEMATICS.}

1548. Ahrendt, H. Bericht über die XII. Hauptversammlung des Vereins zur Förderung des Unterrichts in Mathematik und Physik. Zschr. math. natw. Unt., 34 (1903): 381.

1549. Albrecht, J. A biztositási ismeretek a népiskolában. Versicherungswesen in der Volksschule. Néptanitók Lapja, 42 (1909).

1550. Albrich, K., jr. Die Lehre von der Bewegung fester Körper. Progr. evang. Gymn. A. B. Nagyszeben, 1902.

1551. Appell, P. L'enseignement des sciences et la formation de l'ésprit scientifique. Address at the 37th meeting of " $L$ 'Association française pour l'avancement des sciences." Revue du Mois, 1908; Rev. de l'Ens. des Sciences, 1908; Notes by Ch. Bioche in L'Enseignement Secondaire, 30 (1909).

1552. —_ L'enseignement scientifique à l'Université de Paris. L'Ens. Math., 8 (1906).

1553. — L'enseignement supérieure des sciences. Paris, 1905.

1554. — Les sciences dans l'éducation nationale. Idées Modernes, 1909.

1555. Ascoli, M. Les sciences mathématiques et physiques dans l'enseignement secondaire d'après les conférences du Musée Pédagogique. Revue Générale des Sciences, 15 (1904).

1556. Bartorelli, —. Alcune considerazioni dei calcolo utili per le applicazioni alle scienze sperimentali. Periodico di Matematica, ser. III, 6 (1909).

1557. Bartram, H. The correlation of elementary practical geometry and geography. Reports of the British Subcommission, 6.

1558. Bayern. Zeitgemässe Umgestaltung des mathematischen und naturwissenschaftlichen Unterrichts am humanistischen Gymnasium in Bayern. Natur und Schule, 5 (1906).

1559. Beischer, K. See Calculus.

1560. Bergmann, F. Der Kalender als Lehrmittel. Zschr. Lehrmittelwesen und päd. Literatur, 4 (1909).

1561. Besprechung von Vertretern der angewandten Mathematik in Göttingen, 22-23, III., 1907. Jahresber. D. M. V., 16 (1907).

1562. Bettazzi, R. L'application dans l'enseignement de la mathématique. L'Ens. Math., 2 (1900); and Italian in the Atti Accademia Lucchese, 30 (1900).

1563. Bioche, Ch. See Appell, P. 
1564. Bishop, F. L. Progress in the correlation of physics and mathematics. School Science and Mathematics, 5 (1905): 396.

1565. Branford, B. Measurement and simple surveying, an experiment in the teaching of elementary geometry. Journal of Education (1899-1901).

1566. Breckenridge, W. E. Applied problems. National Education Association of the United States. Journal of proceedings and addresses, 1910. p. 515.

1567. Brown, E. W. The teaching of mechanics. Bulletin of the American Mathematical Society, 14 (1908): 165.

1568. Buchrucker, B. Uber die Sprache der Lehrbücher für Mathematik und Naturwissenschaft. Math. Natw. Blätter, 6 (1909).

1569. Busse, M. Kaufmännisches Rechnen oder Wirtschaftskunde. Zschr. für lateinlose höhere Schulen, 20 (1909). (S. daselbst die Bemerkungen von Dantzig.)

1570. Castelli, G. Sull'insegnamento della matematica attuariale e finanziara nelle scuole professionali inferiori, medie e superiori. Congr. Int. dei Mat. Atti del IV. Roma, 1909.

1571. Ciamberlini, C. and Umani, A. Sul coordinamento degli studio di matematica e di fisica nelle scuole medie. Boll. Mat., 2 (1903).

1572. Clark, C. A. Commercial branches in the high-school curriculum. Educational Review, 37 (1909).

1573. Cobb, H. E. Proposals for reform in the teaching of mathematics and science in the nine-class higher schools of Prussia. School Science and Mathematics, 6 (1906): 708 .

1574. Collins, J. V. Correlation of mathematics with biology, history, and literature. School Science and Mathematics, 5 (1905): 640, 726.

1575. Comstock, C. E. Correlation of mathematics and science. National Education Association of the United States. Journal of proceedings and addresses, 1905. p. 788.

1576. Correlation of the teaching of mathematics and science. Mathematical Gazette, 5 (1909).

1577. Czakó, A. Néhány észrevétel a technikus szempontjából. Einige Bemerkungen vom Standpunkt des Technikers (über die Reform des mathematischen Unterrichts). See Beke-Mikola, under Secondary Schools.

1578. Dalwigk, F. von. Beiträge zur Frage des Unterrichts in angewandter Mathematik an der Universität. Jahresber. D. M. V., 15 (1906).

1579. Daninger, J. Ästhetisches aus der Mathematik und der Physik. Progr. Prag-Altstadt (1906-07).

1580. Danzig, -. See Busse, M.

1581. Darboux, G. Review of E. Fabry: "Traité de mathématiques générales à l'usage des chimistes, physiciens, ingénieurs et des élèves des facultés des sciences" (Paris, 1908) in the Bull. des Sciences Math., 32 (1908).

See also the preface of the work.

1582. Dressler, H. Über bewegliche Modelle für den mathematischen und naturwissenshaftlichen Unterricht. Unterrichtsblätter, 14 (1908).

1583. Dunker, K. Forderungen für den mathematisch-naturwissenschaftlichen Unterricht und seine Vertretung. Unterrichtsblätter, 11 (1905).

1584. — Thesen betreffend die Stellung des mathematisch-naturwissenschaftlichen Unterrichts und seine Vertreter. Unterrichtsblätter, 11 (1905).

1585. Eggar, W. D. The coordination of the teaching of elementary mathematics and physics. School World, 7 (1905).

1586. - The teaching of elementary mechanics. Reports of the British Subcommission, 7 .

1587. Emch, A. Sur quelques exemples mathématiques dans les sciences naturelles. L'Ens. Math., 12 (1910): 114. 
1588. L'enseignement des sciences mathématiques et des sciences physiques. Conf. du Musée Pédag., 6 (1904). (Addresses by L. Liard, H. Poincaré, G. Lippmann, L. P. Poincaré, P. Langevin, E. Borel, F. Marotte.)

1589. Fabry, E. See Darboux, G.

1590. Faulland, J. Methodische Behandlung der Zinseszinsrechnung und Rentenrechnung. Blätter für das Gymn. Schulw., 41 (1905): 206.

1591. Fick, A. Über die Vorbildung zum Studium der Medizin. Ges. Schriften Würzburg, 4 (1906).

1592. Filon, L. N. G. The relation of mathematics and physics. Reports of the British Subcommission, 2.

1593. Fischer, K. T. Der naturwissenschaftliche Unterricht in England. Leipzig, 1901.

1594. Förster, w. Der mathematisch-naturwissenschaftliche Unterricht. Vortrag. Lebensfragen und Lebensbilder, Berlin, 1902.

1595. Forsyth, A.R. Discussion on the teaching of elementary mechanics. British Association meeting at Johannesburg, 1905. Edited by A. R. Forsyth. London, 1906.

1596. Francis, W. A. Mathematics and the coordination of mathematics and physics in secondary schools. School Review, 11 (1903): 811.

1597. Fricke, K. Über die heutige Lage des mathematisch-naturwissenschaftlichen Unterrichts an den höheren Schulen. Phys. Zschr., 5 (1909); abgedruckt in den Wangerin'schen Verhandlungen der Gesellsch. D. N. und $\ddot{A}$.

1598. Friemel, R. Wie sind die angewandten Rechenaufgaben für die einzelnen Stufen zweckmässig anzuwählen und anzuordnen? Aus der Schule, Leipzig, 16 (1905).

1599. Galdéano, Z. G. L'enseignement scientifique en Espagne. L'Ens. Math., 4 (1902).

1600. Geissler, K. Methodische Wege für dauernden Zusammenhang der Mathematik mit den übrigen Lehrfächern. Unterrichtsblätter, 15 (1909).

1601. Gerhardt, R. Die Lebensversicherung im mathematischen Unterricht. Unterrichtsblätter, 15 (1909); Zeitschrift für die gesamte Versicherungswissenschaft, 12 (1912); Berlin, 1912.

1602. _ Zur Anwendung der Zinseszinsrechnung im Unterricht. Unterrichtsblätter, 15 (1909).

1603. Giebel, K. Die Handarbeit an den höheren Knabenschulen. Progr. Oberrealschule Zeitz (1908-9).

1604. Glauner, Th. Die Bedeutung der Mathematik und der Naturwissenschaften für die allegmeine Bildung. Progr. Melanchton Gymn. Wittenberg, 1907.

1605. Goldziher, K. Alkalmazott matematika és fizika az egyetemen. Angewandte Mathematik und Physik auf der Unversität. Magyar Egyetemi Szemle, 1 (1901).

1606. — Reformtörekvések a reális tárgyak oktatása terén. Reformbestrebungen im Gebiete des Unterrichts der realen Fächer. O. K. T. K., 39 (1905-06). See also Technical Schools.

1607. Gouilly, A. Sur l'enseignement élémentaire de la mécanique. L'Ens. Math., 6 (1904): 12, 219.

1608. Greenhill, Sir G. Teaching of mechanics by familiar applications on a large scale. Verhandl. des III. Int. Math. Congr. in Heidelberg (1904). Leipzig, 1905.

1609. Grosse, W. Über eine praktische Rechnungsaufgabe der Feldmesskunst. Zschr. math. natw. Unt., 35 (1904): 33.

1610. — Vorbemerkungen zu neuen Lehrplänen in Mathematik und Physik. Zschr. math. natw. Unt., 41 (1910): 256. 
1611. Guerritore, G. Utilità delle considerazioni geometriche nella discussione dei problemi di matematica finanziaria. Il Pitagora, 1910.

1612. Gutzmer, A. (a) Der mathematische und naturwissenschaftliche Unterricht an den Reformschulen; (b) an den sexklassigen Realschulen; (c) an den höheren Mädchenschulen. Vorschläge der Stuttgarter Versammlung, 1906: Gesamtbericht.

1613. Hadamard, J. Les sciences dans l'enseignement secondaire. La Science au $\mathrm{XX}^{\mathrm{me}}$ siècle, 1 (1903).

1614. Hall, E. H. Mathematics and the coordination of mathematics and physics in secondary schools. School Reveiw, 11 (1903): 807.

1615. Halsted, G. B. Measurement. School Science and Mathematics, 8 (1908): 208.

1616. Harper, G. A. Discussion on the report of the commission on real applied problems in algebra and geometry. School Science and Mathematics, 10 (1910).

1617. Hauck, G. Über den Universitätsunterricht in angewandter Mathematik. Jahresber. D. M. V., 8 (1900).

1618. Hecht, W. Der mathematische und physikalische Unterricht in den drei oberen Klassen unserer humanistischen Gymnasien. Blätter für das Gymn. Schulw., 36 (1900): 34 .

1619. Hermes, J. Geschwindigkeitslehre in der Schule. Jahresber. Realgymn. Osnabrück, 1901.

1620. Hessen. Über die Reform des mathematischen und naturwissenschaftlichen Unterrichts im Grossherzogtum Hessen. Gesuch der Ortsgruppe Darmstadt des Realschulmännervereins; in J. W. Volsing's Article in Monatschrift für Natw., 2 (1909); and Lietzmann's Rubrik Zschr. math. natw. Unt., 40 (1909).

1621. Die heutige Vertretung der Interressen des mathematischen und naturwissenschaftlichen Unterrichts durch die Fachlehrer selbst. Zschr. für lateinlose höhere Schulen, 19 (1908).

1622. Höckner, G. Die Mathematik in der Lebensversicherung. Klein's Abhandlungen, III., 9 .

1623. Hoffmann, B. Astronomie, Vermessungswesen, mathematische Geographie an den höheren Schulen. Klein's Abhandlungen, III, 4.

1624. Höfler, A. Das Mathematische im physikalischen Unterricht. Zschr. phys. chem. Unt., 18 (1905).

1625. Huber, - Mathematische Behandlung der Elektronentheorie im gymnasialen Unterricht. Baseler Verhandlungen.

1626. Hurst, G. H. G. Mathematics and physics in public schools. Nature, 63 (1900-1901): 370 .

1627. Jackson, C. S. The teaching of mathematics and physics. Mathematical Gazette, 3 (1904-06): 75.

1628. Jackson, D. C. Desirable product from the teacher of mathematics, the point of view of an engineering teacher. School Science and Mathematics, 5 (1905): 67.

1629. Jacobs, A. Was leistet der Mathematikunterricht für die Erziehung zur Wissenschaft? Zschr. math. natw. Unt., 39 (1908): 625.

1630. Jacquemir, A. L'enseignement des sciences en Amérique. Rev. de l'Ens. Sciences, 2 (1908).

1631. Jamieson, W. R. Educational method with special reference to the teaching of mathematical and physical sciences. Australian Association for the Advancement of Sciences, 11 (1907).

1632. Johanneson, P. Eine Bemerkung über physikalisches Rechnen. Zschr. math. natw. Unt., 42 (1911). 
1633. Jung, P. Zur Behandlung der Versicherungslehre im Unterricht. Zschr. math. natw. Unt., 34 (1903): 226.

1634. Junge, G. Nochmals Astronomie auf der Schule. Monatschrift für höhere Schulen, 8 (1909).

1635. Kinkaid, E. M. Banking and other business arithmetic in the schoolroom. School Journal, 76 (1908).

1636. Kissel, F. Zum Unterricht in der Zins-Zinseszins-und RentenrechnungProgr. Hum. Gymn. Kaiserslautern, 1908.

1637. Kleefstra, J. Over het onderwijs in de wiskunde. Haarlem, 1909.

1638. Klein, F. Allgemeines über angewandte Mathematik. In Klein-Riecke, Über angewandte Mathematik und Physik.

1639. - Bemerkungen zum mathematischen und physikalischen Unterricht. Wangerin'sche Verhandlungen; mit dem Titel: "Bericht an die Breslauer Naturforscherversammlung über den Stand des mathematischen und physikalischen Unterrichts an den höheren Schulen" in Klein-Schimmack, Vorträge, under Secondary Schools.

1640. — Mathematik und Naturwissenschaft. Universität und Schule, Leipzig, 1907.

1641. Probleme des mathematisch-physikalischen Hochschulunterrichts. Jahresber. D. M. V., 14 (1905); Zschr. math. natw. Unt., 36 (1905): 451; and in Klein-Schimmack, Vorträge, under Secondary Schools.

1642. U Über die Aufgabe der angewandten Mathematik, besonders über die pädagogische Seite. Verhandl. des III. Int. Math. Congr. in Heidelberg, Leipzig, 1905.

1643. Z Zur Besprechung des mathematisch-naturwissenschaftlichen Unterrichts auf der nächsten Naturforscherversammlung zu Breslau. Jahresber. D. M. V., 13 (1907).

See also Colleges and Universities.

1644. - and Riecke, E. Neue Beiträge zur Frage des mathematischen und physikalischen Unterrichts an den höheren Schulen. Leipzig, 1904.

1645. — Über angewandte Mathematik und Physik in ihrer Bedeutung für den Unterricht an den höheren Schulen. Nebst Erläuterung der bezüglichen Göttinger Universitätseinrichtungen. Leipzig, 1900; Lehrp. und Lehrg., 17 (1901).

1646. Knabl, E. Konstruktive Lösung elementarer astronomischer Aufgaben als Beitrag zur Himmelskunde und angewandten Geometrie an Mittelschulen. Progr. Stockerau (1903-04).

1647. Krimphoff, w. Die Grundzüge des bankmässigen Rechnens. Progr. Kgl. Gymn. Warendorf, 1908.

1648. Laparevicz, A. Bibliografia nauczania przedmiotów matematyczno-fizycznych w zakresie šrednim. Wiadomości Matematyczne, 14 (1910).

1649. Lemoine, J. Les mathématiques et la physique dans les sections du II. cycle. Rev. de l'Ens. des Sciences, 2 (1908).

1650. Liard, L. L'enseignement des sciences mathématiques et des sciences physiques. Circulaire. Rev. de l'Ens. des Sciences, 1 (1907).

1651. - Les sciences dans l'enseignement secondaire. Conf. du Musée Pédag., 6 (1904).

1652. Lietzmann, w. Deutscher Ausschuss für mathematischen und naturwissenschaftlichen Unterricht und Internationale Mathematische Unterrichtskommission. Zschr. für Hochschulpäd., 2 (1911).

1653. - Mathematische und naturwissenschaftliche Schülerbibliotheken. Päd. Arch., 53 (1910); mit Bemerkungen von J. Moeller und H. O. Zimmer daselbst, 54 (1911). 
1654. Lietzmann, w. Über die Reformbewegung auf dem Gebiete des mathematischen und naturwissenschaftlichen Unterrichts. Zschr. math. natw. Unt., 39 (1908): 646.

1655. — Zur Reformbewegung auf dem Gebiete des mathematischen und naturwissenschaftlichen Unterricht. Regular department in Zschr. math. natw. Unt., since 1909.

1656. Lilienthal, R. von. Über die Verwertung der politischen Arithmetik im mathematischen Unterricht. Unterrichtsblätter, 17 (1911).

See also Colleges and Universities.

1657. Long, E. Discussion. Correlation of algebra, geometry, and physics. Educational Review, 24 (1902): 309.

1658. Lorenz, H. Der Unterricht in angewandter Mathematik und Physik an den deutschen Universitäten. Jahresber. D. M. V., 12 (1903).

1659. Ludwig, F. Mathematische Schulaufgaben aus der Naturgeschichte. Natur und Schule, 7 (1907).

1660. Mach, A. Die Rentenrechnung ohne geometrische Progressionen. Zschr. für das Realschulw., 29 (1904): 211.

1661. McIntosh, J. The mathematics of elementary chemistry. School Science and Mathematics, 7 (1907).

1662. Marotte, $\mathbf{F}$. L'enseignement des sciences mathématiques et physiques dans l'enseignement secondaire en Allemagne. Conf. du Musée Pédag., 1904, 1905.

1663. - Les mathématiques et la physique dans les sections littéraires du second cycle. Rev. de l'Ens. des Sciences, 2 (1908).

1664. Mathematical Association and Association of Public School Science Masters. Report of the joint committee on the correlation of science and mathematics teaching. Mathematical Gazette, 5 (1909-10): 145.

1665. Maurer, A. Der mathematisch-naturwissenschaftliche Unterricht. Südwestdeutsche Schulblätter, 31 (1904).

1666. Medicin. Welche Mittelschulbildung ist für das Studium der Medicin wünschenswert? Ärzt. Verein München, 17. Nov. 1909. Münch. Medic. Wochenschrift, 1910.

1667. Mikola, S. A középiskolai mathematikai és természettudományi tanitás reformja. Reform des mathematischen und naturwissenschaftlichen Unterrichts der höheren Schule. Urania, 1909.

1668. Millis, J. F. Real applied problems in algebra and geometry. School Science and Mathematics, 9 (1909); 10 (1910).

1669. - The real problem movement in its relation to the teaching of geometry and algebra in the secondary schools. National Education Association of the United States. Journal of proceedings and addresses, 1909. p. 519.

1670. Müller, F. Welche Mittelschulbildung ist für das Studium der Medicin wünschenswert? Zschr. math. natw. Unt., 42 (1911); Schriften des Deutschen Auschusses, Leipzig, 1911.

1671. Myers, G. W. A practical presentation of some problems correlating mathematics and physics. School Review, 11 (1903): 232.

1672. Neurath, O. Die allgemeine Einführung des volkswirtschaftlichen und staatsbürgerlichen Unterrichts. Kultur und Fortschritt, 168 (1907).

1673. Nitsche, O. Die Behandlung versicherungs-mathematischer Aufgaben im Unterricht. Lehrp. und Lehrg., 23 (1907): 294.

1674. Het onderwijs in de beginselen de Wiskunde in Italië. Wiskundig Tijdschrift, $2(1905-06)$.

1675. Packard, J. C. Mathematics and the coordination of mathematics and physics in secondary schools. School Review, 11 (1903): 798. 
1676. Pahl, F. Literaturbericht über Mathematik und Physik. Pädagogischer Literaturbericht, Berlin.

1677. Perry, J. The correlation of the teaching of mathematics and science. Nature, 79 (1908-09): 143; Mathematical Gazette, 5 (1909).

1678. —_ L'enseignement mathématique dans ses rapports avec l'enseignement des sciences. L'Ens. Math., 11 (1909): 136.

1679. Peters, F. N. Scientific and mathematical teaching in the future. School Science and Mathematics, 9 (1909).

1680. Pietzker, F. Der exaktwissenschaftliche Unterricht in der SchulreformBewegung. Unterrichtsblätter, 9 (1903).

1681. Pologne. L'enseignement des mathématiques et de la physique dans les écoles privées de Pologne. Rapport du Cercle mathematico-physique de Varsovie. L'Ens. Math., 13 (1909): 299.

1682. Postelmann, E. Zur Reform des mathematischen und naturwissenschaftlichen Unterrichts auf dem Gymnasium. Progr. Bartenstein (1910-11).

1683. Presler, O. Bericht über die Hauptversammlung des Vereins zur Förderung des mathematischen und naturwissenschaftlichen Unterrichts. Zschr. für lateinlose höhere Schulen, 18 (1907).

1684. _ Die Ergebnisse der Dresdener Tagung des Vereines zur Förderung des Unterrichts in Mathematik und in den Naturwissenschaften. Zschr. für lateinlose höhere Schulen, 18 (1907).

1685. Pressland, A. J. The reform of mathematical and science teaching in Germany. Nature, 88 (1910-11): 147.

1686. Pyle, L. A correlation of mathematics and physics. School Science and Mathematics, 10 (1910): 586.

1687. Reformvorschläge für den mathematischen und naturwissenschaftlichen Unterricht. Entworfen von der Unterrichtskommission der Gesellschaft Deutscher Naturforscher und Ärzte. I. (Meran, 1905), Zschr. math. natw. Unt., 36 (1905): 533; II. (Stuttgart, 1906), ib. 37 (1906): 409; III. (Dresden, 1907), ib. 38 (1907): 401.

See also Verhandlungen, edited by A. Wangerin.

1688. Reinhardt, C. Ziele des mathematisch-naturwissenschaftlichen Unterrichts am Gymnasium. Neue Jahrb., 2 (1901).

1689. Richter, O. Zur Vertiefung des Unterrichts in Mathematik und den Naturwissenschaften. Neue Jahrb., 10 (1907).

1690. Rieh1, A. Humanistische Ziele des mathematischen und naturwissenschaftlichen Unterrichts. Berlin, 1909.

1691. Roubault, _- Pour l'enseignement du travail manuel. Rev. de l'Ens. des Sciences, 2 (1908).

1692. Ruault, _. Enseignement de la physique et des mathématiques dans les classes supérieures. Rapport presenté au Congrès de 1910 de la Fédération des Collèges. Rev. de l'Ens. des Sciences, 5 (1911).

1693. Rudel, E. Die Fühlung zwischen Mathematik und ihren Anwendungsgebieten im Unterricht. Bay. Zschr. für Realschulw., 15 (1907).

1694. Rundschreiben des Pariser Schulbezirkes an die Bezirksinspektoren (inspecteurs d'Académie), Direktoren und Professoren der Mathematik und Physik seines Amtsbezirkes. Zschr. für das Realschulw., 32 (1907): 577.

1695. Schilling, G. Verhandlungen über den mathematisch-naturwissenschaftlichen Unterricht auf der Breslauer Versammlung Deutscher Naturforscher und Ärzte. Zschr. für das Österr. Gymnasialw., 56 (1905).

1696. Schmidt, O. Beiträge zu einem Lehrplan der realistischen Fächer. Päd. Bausteine, Nr. 2, Berlin.

1697. — Koncentration des Unterrichts auf realistischer Grundlage. Päd. Bausteine, Nr. 13, Berlin. 
1698. Schotten, $\mathbf{H}$. Der Deutsche Ausschuss für mathematischen und naturwissenschaftlichen Unterricht. Zschr. math. natw. Unt., 39 (1908): 537.

1699. — Der mathematisch-naturwissenschaftliche Unterricht an den sechsklassigen Realschulen. Zschr. math. natw. Unt., 37 (1906): 235.

1700. — Zur Reform des mathematisch-naturwissenschaftlichen Unterrichts. Zschr. math. natw. Unt., 36 (1905): 226, 311, 380.

1701. Schülke, A. Die Behandlung von Dach- und Brücken-constructionen im Unterricht. Zschr. phys, chem. Unt., 14 (1901).

1702. - Finanzpolitik und die Erziehung zum öffentlichen Leben. Blätter höh. Schulw., 25 (1908).

1703. — Lebensversicherungsrechnungen im Unterricht. Unterrichtsblätter, 9 (1903).

1704. Schwartz, A. Der geodätische Kursus der Oberrealschule an der Waitzstrasse in Kiel. Progr. Oberrealschule an der Waitzstrasse in Kiel, 1906.

1705. Slater, C. H. What physics asks of mathematics. School Science and Mathematics, 6 (1906): 468.

1706. Smith, D. E. The question of problems in elementary mathematics. Educational Review, 31 (1906): 300.

1707. Stäckel, P. Angewandte Mathematik und Physik an den deutschen Universitäten. Jahresber. D. M. V., 13 (1909).

1708. - Angewandte Mathematik in den preussischen Universitäten. Monatschrift für höhere Schulen, 3 (1904).

1709. Sturm, —. Unser Geld; Lektion für die Oberstufe. Arch. für Volksschullehrer, 11 (1908).

1710. Szabó, G. A matematika és fizika vonatkozásai. Wechselbeziehungen der Mathematik und Physik. Beke-Mikola, Sammlung, under Secondary Schools.

1711. Tešar, I. Beispiele aus der Mechanik. 39. Jahresber. deutschen Landesoberrealschule Prossnitz (1903-04).

1712. — Beiträge zum Unterrichte in der Hydrodynamik. Zschr. für das Realschulw., 33 (1908): 333.

1713. Thiemayer, K. Die Mathematik in ihrer Anwendung auf Versicherungswesen. Progr. Städt. Realprogymn. Papenburg, 1902.

1714. Thieme, H. Wirkung der wissenschaftlichen Ergebnisse auf den Unterricht in den Elementen der Mathematik. Verhandl. des III. Int. Math. Congr. in Heidelberg, Leipzig, 1905.

1715. Thurston, E. I. Mathematics in commercial work. School Review, 11 (1903): 585; National Education Association of the United States. Journal of proceedings and addresses, 1903. p. 726.

1716. Tiebe, A. Die mathematisch-naturwissenschaftlichen Lehrpläne der Unterrichtscommission der Gesellschaft Deutscher Naturforscher und Ärzte. Zschr. für lateinlose höhere Schulen, 18 (1907).

1717. Timerding, H. E. Die kaufmännischen Aufgaben im mathematischen Unterricht der höheren Schulen. Klein's Abhandlungen, III, 5.

1718. _ Die Mathematik in den physikalischen Lehrbüchern. Klein's Abhandlungen, III, 2.

1719. Tower, M. E. How much mathematics should the physics teacher teach? National Education Association of the United States. Journal of proceedings and addresses, 1904. p. 516.

1720. Treutlein, P. Die Verhandlungen des Jahres 1905 zur Hebung des mathematisch-naturwissenschaftlichen Unterrichts mit besonderer Berücksichtigung der Reformschule. Vortrag. Zschr. des Ver. deutscher Ing., 1906.

1721. Umani, A. See Ciamberlini, C.

1722, Unger, F. Gewerbliches Rechnen. Zschr. math. natw. Unt., 37 (1906). 
1723. Upshall, C. W. Weather observation in schools. Practical Teacher, 29 (1908).

1724. Verein deutscher Ingenieure. Bericht zur Frage des für höhere wissenschaftliche Laufbahn vorbereitenden Schulunterrichts. (1904, 1905.)

1725. Vogel, E. Die Grundsätze der stereographischen Projektion." (Für den Unterricht in der VII. Realschulklasse.) Zschr. für das Realschulw., 34 (1909): 598 .

1726. Walckling, R. Bericht über die XIII., XIV., XVI., XVII. Hauptversammlung des Vereins zur Förderung des Unterrichtes in der Mathematik und den Naturwissenschaften. Zschr. math. natw. Unt., 35 (1904): 359, 443; 36 (1905): $385,465,582$; 38 (1907): 307, 369; 39 (1908): 197, 309, 401.

1727. Wallentin, J. G. Über die Methodik des physikalischen Unterrichts. Zschr. für das Österr. Gymnasialw., (1902): 698.

1728. Wangerin, A. Verhandlungen der Naturforscher-Versammlung über den naturwissenschaftlichen und mathematischen Unterricht an den höheren Schulen. Herausgegeben von A. Wangerin. Verhandl. der G. D. N.u. Ä., Leipzig (1904-07).

1729. Weitere und engere Verbände auf dem Gebiete des mathematischen und naturwissenschaftlichen Unterrichtes. Unterrichtsblätter, 14 (1908).

1730. Wernicke, A. Die kulturelle Bedentung der mathematisch-naturwissene schaftlichen Forschung. Päd. Arch., 45 (1904).

1731. Westman, J. Meteorologien i realskolan och gymnasiet. Ped. Tidskr., 1908.

1732. White, R. D. What mathematics can do for physics. School Science and Mathematics, 6 (1906): 287.

1733. Wieleitner, H. Über die mathematisch-physikalische Lehraufgabe und diAusbildung der Fachlehrerim Königreich Bayern. Zschr. math. natw. Unt., 34 (1903): 89.

1734. Witting, A. Die Abteilung für mathematischen und naturwissenschaftlichen Unterricht auf der $82^{\text {er }}$ Versammlung Deutscher Naturforscher und Ärzte in Königsberg. Päd. Arch., 53 (1911).

1735. _ B Bericht über die XIX ${ }^{\text {te }}$ Hauptversammlung des Vereins zur Förderung des mathematischen und naturwissenschaftlichen Unterrichts zu Posen, 1910. Päd. Arch., 52 (1910); Münster, i. W., 1911, Päd. Arch., 53 (1911).

1736. Woodruff, E. C. Algebra from the standpoint of the teacher of physics. School Review, 11 (1903): 798.

1737. Wormell, $\mathbf{R}$. Unstable questions in the teaching of elementary science. Educational Times, 1900.

1738. Young, J. W. A. The movement in Prussia for the reorganization of the instruction in mathematics and the natural sciences in the secondary schools. Science, 1906.

\section{THE VALUE OF THE HISTORY OF MATHEMATICS IN TEACHING.}

1739. Braunschweig, R. Über die Berücksichtigung des Historischen im mathematischen Unterricht. Progr. Staatsrealschule Böhmisch-Leipa, (1908-09).

1740. Broomell, B. L. Mathematical historical material from the East. Mathematics Teacher, 1 (1908-9): 16.

1741. Gebhardt, M. Geschichte der Mathematik an den höheren Schulen. Klein's Abhandlungen, III, 6.

1742. — Das Geschichtliche im mathematischen Unterricht mit besonderer Berücksichtigung des humanistischen Gymnasiums. Progr. 47. Vitzthum'sches Gymn. Dresden, 1908; Päd. Arch., 52 (1910).

1743. Miller, G. A. The study of the history of mathematics. School Science and Mathematics, 6 (1906): 294. 
1744. Richter, A. Der Unterricht in der Geschichte der Mathematik. Zschr. math. natw. Unt., 40 (1909): 87.

1745. Servaty, J. Some points in the history of Greek mathematics which are useful in secondary teaching. School Science and Mathematics, 5 (1905): 553,606 .

\section{PREPARATION OF TEACHERS OF MATHEMATICS.}

1746. Arldt, - Die Vorbildung der sächsischen Realschullehrer. Zschr. für lateinlose höhere Schulen, 18 (1907).

1747. Ausbildung von Lehramtskandidaten der Mathematik in Physik an technischen Hochschulen. Leitsätze der Darmstädter Versammlung des Vereins deutscher Ingenieure, 6 (1908): 5; Jahresber. D. M. V., 17 (1908).

1748. Beke, M. A matematikai tanárképzés tekintettel a reformtörekvésekre. Die Lehrerausbildung in der Mathematik mit Rücksicht auf die Reformbestrebungen. See Beke-Mikola, Sammlung, under Secondary Schools.

1749. Berak, J. Über den mathematischen Unterricht an den Lehrerbildungsanstalten. Progr. Lehrerbildungsanst. Kremsier.

1750. Bock, A. See Treutlein, under Elementary Schools.

1751. Böhme, B. Das Seminar als höhere Schule; Abschnitt Mathematik. Leipzig, 1910.

1752. Bryan, G. H. The uses of mathematics and the training of teachers. Mathematical Gazette, 4 (1908).

1753. Cauer, P. Die Lehrerausbildung in Bayern und Preussen. Neue Jahrb., 13 (1910).

1754. Chabot, C. The professional training of teachers in France. Congress of Arts and Science, St. Louis Exposition, 1904, vol. 8. Boston, 1907.

1755. Chatelet, A. Le laboratoire d'enseignement mathématique de l'École Normale Supérieure de Paris. L'Ens. Math., 11 (1909); Lietzmann's Rubrik in Zschr. math. natw. Unt., 40 (1909).

1756. Coar, H. L. The teacher of mathematics. School Science and Mathematics, 7 (1907): 282.

1757. Conti, A. Relazione nell'insegnamento della matematica nelle scuole normali. Congr. dei Prof. di Mat. Atti del II. Livorno, 1902.

1758. _ Sulla iniziazione alle matematiche e sulla preparazione matematica dei maestri elementari in Italia. Vortrag auf dem IV. Int. Math. Congr. in Rom, 1908; Boll. Mat., 9 (1910).

1759. Cours de vacances pour les maîtres de l'enseignement secondaire en Autriche. L'Ens. Math., 7 (1909).

1760. Courses for the training of teachers of mathematics. Teachers College, Columbia University, New York; Announcements of the Department of Mathematics. Similar announcements of the University of Chicago and several other American universities.

1761. Cramer, H. See Secondary Schools, and Elementary Schools under Treutlein.

1762. Czuber, E. Angewandte Mathematik als Gegenstand der Lehramtsprüfung. Zschr. für das Realschulw., 25 (1900): 17.

1763. Dahlgren, H. See Elementary Schools.

1764. Dannemann, F. Der naturwissenschaftliche Unterricht auf praktischheuristischer Grundlage. Kap. E. Über die Vorbildung der Lehrer für den praktisch-heuristischen Unterricht. Hannover, 1908.

1765. Dékány, M. A tanitóképzö-intézetek mennyiségtani oktatásának sociális vonatkozásai. Sociale Beziehungen des mathematischen Unterrichts an Lehrerseminaren. Magyar Tanitóképzö, 24 (1909).

$47398^{\circ}-12-6$ 
1766. Dressler, H. Die gegenwärtige Lage des mathematischen und naturwissenschaftlichen Unterrichts an den sächsischen Seminaren. Zschr. math. natw. Unt., 39 (1908): 406.

See also Elementary Schools.

1767. Eickhoff, R. Die seminaristisch gebildeten Lehrer an den höheren Lehranstalten. Blätter höh. Schulw., 26 (1909).

1768. Escherich, G. and Jacob, J. Die Ausbildung der Mittelschullehramtskandidaten in Mathematik. Österr. Mittelschule, 24 (1910); Deutschöst. Mittelschultag, 10.

1769. Fehr, H. Les études mathématiques à l'Ecole Normale de l'Université Columbia de New York. L'Ens. Math., 6 (1904): 313.

1770. Ferienkurs in Mathematik, Physik, Chemie, und Erdkunde für Oberlehrer und Oberlehrerinnen an höheren Lehranstalten für die weibliche Jugend, 4-16, X., 1909, in Göttingen. Frauenbildung, 10 (1909); Lietzmann's Rubrik in Zschr. math. natw. Unt., 41 (1910). See also the same Rubrik, 40 (1909), 539.

1771. Fischer, K. T. Vorschläge zur Hochschulausbildung der Lehramtskandidaten für Physik. Natur und Schule, 6 (1907).

1772. Fricke, K. Die geschichtliche Entwickelung des Lehramts an höheren Schulen. Leipzig, 1903.

1773. Friedel, V. H. Traitement des instituteurs et des institutrices à l'étranger. Conf. du Musée Pédag., 3 (1903).

1774. Fries, W. Die neue Ordnung für die praktische Ausbildung der Kandidaten des höheren Lehramts in Preussen. Lehrp. und Lehrg., (1909).

1775. Fuger, J. See Secondary Schools.

1776. Gajdeczka, J. Mathematischer Lehrstoff für Lehramtskandidaten der Bürgerschulen. Wien, 1909.

1777. Geck, E. See Secondary Schools, and Elementary Schools under Treutlein.

1778. Gendame de Bévotte, —. L'école normale supérieure et la préparation à l'enseignement secondaire. Rev. de l'Ens. des Sciences, 3 (1909).

1779. Goldziher, $\mathbf{K}$. Der mathematische Unterricht an den Lehrerbildungsanstalten. Berichte der ungarischen Subkommission.

1780. Goller, —. Das Seminarjahr für Lehramtskandidaten der Mathematik und Physik. Bay. Zschr. für Realschulw., 17 (1909).

1781. Gutzmer, A. La préparation des candidats à l'enseignement des sciences mathématiques et naturelles. L'Ens. Math., 10 (1908).

1782. Hensing, H. See Treutlein, under Elementary Schools.

1783. Höfler, A. Mathematik und Lehrerausbildung. Päd. Blätter, 39 (1910).

1784. Holzmüller, G. Bemerkungen über den Unterricht und die Lehramtsprüfung in der angewandten Mathematik; 2 Artikel. S. die 2 Artikel von Ebner; Jahresber. D. M. V., 14 (1905); Zschr. für lateinlose höhere Schulen, 17 (1906).

1785. Jacob, J. See Escherich, G.

1786. Josephson, O. Die Mathematik an den schwedischen höheren Lehrerseminaren. Berichte der schwedischen Subkommission.

1787. Kabisch, O. Die Neuordnung der höheren Mädchenschulbildung in Preussen vom Standpunkt des Lehrerseminars betrachtet. Päd. Blätter, 38 (1909).

1788. Kerschensteiner, G. See Treutlein, under Elementary Schools.

1789. Klein, F. Aktuelle Fragen der Lehrerbildung. Vortrag auf der Versammlung des Vereins zur Förderung des mathematischen und naturwissenschaftlichen Unterrichts. Schriften des Deutschen Ausschusses, Leipzig, 1911. 
1790. Klein, F. Allgemeine Ausführungen zu den Vorschlägen der Unterrichtskommission über Lehrerausbildung. Verhandl. der G. D. N. u. ̈̈., Leipzig, 29 (1907).

1791. — La préparation des candidats à l'enseignement des sciences mathématiques et naturelles. L'Ens. Math., 10 (1908).

1792. — Über die geplanten mathematisch-naturwissenschaftlichen Vorbereitungskurse für Mittelschullehrer an der Universität Göttingen. Päd. Blätter, 1910.

1793. — Über die preussische Lehrerbildung. Päd. Blätter, 40 (1911).

1794. — and Gutzmer, A. Vorschläge für die wissenschaftliche Ausbildung der Lehramtskandidaten der Mathematik und Naturwissenschaften. Verhandl. der G. D. N. u. Ä., Leipzig, 1907; Vortrag auf dem IV. Int. Math. Congr. in Rom., 1908.

1795. Kleinpeter, H. Vorbildung der Lehrer. Päd. Arch., 44 (1902).

1796. Konrath, Th. Der mathematische Unterricht an den Bildungsanstalten für Lehrer und Lehrerinnen. Berichte über den mathematischen Unterricht in Österreich, 2.

1797. Krause, M. Über die Ausbildung von Lehrern der mathematisch-naturwissenschaftlichen Richtung an der technischen Hochschule in Dresden. Vortrag. Unterrichtsblätter, 13 (1907).

1798. Krebs, W. Der Strassburger Ferienkursus für Lehrer der Naturwissenschaften und Mathematik, 14-23 Oktober, 1901. Zschr. math. natw. Unt., 33 (1902): 208, 302.

1799. Kürschák, J. Die Ausbildung der ungarischen Mittelschulprofessoren. Berichte der ungarischen Subkommission.

1800. Langlois, C. V. La préparation professionelle à l'enseignement secondaire. Conf. du Musée Pédag., 2 (1902).

1801. Lehrplan des Lübecker Lehrerseminars, 1909. Lietzmann's Rubrik in Zschr. math. natw. Unt., 1911.

1802. Lehrplan in Mathematik für sächsische Seminare. Zschr. math. natw. Unt., 33 (1902): 413 .

1803. Lilienthal, R. von. See Colleges and Universities.

1804. Lietzmann, w. Reformvorschläge für die wissenschaftliche Ausbildung der Lehramtskandidaten der Mathematik und Physik. Math. Natw. Blätter, 4 (1907).

See also Elementary Schnols.

1805. Loos, J. Die praktische Vorbildung für das höhere Lehramt in Österreich. Zum Schluss, eine Bibliographie über Schriften allgemeiner Tendenz dieses Gegenstandes. Berichte über den mathematischen Unterricht in Österreich, 4 .

1806. Lorey, w. Bemerkungen über die geplante Zulassung von Lehrern an der Universität Göttingen. Korrespondenzblatt für den akademisch gebildeten Lehrer, 18 (1910).

1807. Makchéev, M. Notice sur les cours pour la préparation des maîtres des Corps de Cadets. Rapports de la sous-commission russe, 5 .

1808. Martinak, E. Zur pädagogischen Vorbildung für das Lehramt an Mittelschulen. Zschr. für das Österr. Gymnasialw., 1904.

1809. Miller, G. A. Group theory for teachers of elementary mathematics. School Science and Mathematics, 6 (1906): 752.

1810. Moore, M. E. La préparation professionelle des instituteurs en Angleterre. Revue Pédagogique, 1 (1908).

1811. Morsch, H. Das höhere Lehramt in Deutschland und Österreich, mit einem Ergänzungsband. Leipzig, 1905, 1907. 
1812. Müller, F. Welche Bedeutung hat für den Lehrer der Mathematik die Kenntniss der Geschichte, Literatur und Terminologie seiner Wissenschaft? Zschr. für das Gymnasialw., 57 (1903): 801.

1813. - and Schiffner, F. See Schiffner, F.

1814. Muthesius, K. Grundsätzliches zur Volksschullehrerbildung. Schriften des Deutschen Ausschusses, Leipzig, 1911.

1815. Nath, M. Über die wissenschaftliche Ausbildung der Lehramtskandidaten der Mathematik und Naturwissenschaften. Blätter höh. Schulw., 25 (1908).

1816. — Die Vorbildung für das höhere Lehramt, insbesondere in der Mathematik und den Naturwissenschaften. Neue Jahrb., 10 (1907).

1817. Pincherle, S. See Colleges and Universities.

1818. Pittarelli, G. Modificazioni da introdursi nell' insegnamento matematico superiore per la preparazione degli insegnanti secondari. Congr. dei Prof. di Mat. Atti del II. Livorno, 1902.

1819. Plath, J. See Müller, H.

1820. Per la preparazione degli insegnanti. Boll. Mat., 5 (1906).

1821. Prüfungsordnung für das Lehramtsexamen in der angewandten Mathematik. 1900.

See Zschr. math. natw. Unt. 32 (1901).

1822. Prüfungsordnung für die Kandidaten des höheren Lehramts in Preussen. Halle, 1903.

1823. Ratschläge für diejenigen Studierenden welche sich auf das höhere Lehramt vorbereiten und einen Teil ihrer Studienzeit auf der technischen Hochschule verbringen. Kgl. Techn. Hochschule Aachen, 1905.

1824. Reinhardt, R. Über die Wünsche der Fachlehrer hinsichtlich des Hochschulunterrichts für künftige Lehramtskandidaten in Mathematik und Physik. Referat bei der Dresdener Hauptsitzung des Vereines zur Förderung des Unterrichts in der Mathematik und in den Naturwissenschaften. Unterrichtsblätter, 13 (1907).

1825. Ruediger, W. E. Agencies for the improvement of teachers in service. Washington, D. C., 1912. 157 p. $8^{\circ}$. (U. S. Bureau of Education. Bulletin, 1911, no. 3.)

1826. Ruska, J. Die wissenschaftliche Ausbildung der Lehramtskandidaten der Mathematik und Naturwissenschaften. Päd. Arch., 51 (1909).

1827. Scherrer, F. R. Der mathematische Unterricht an den Lehrer-Lehrerinnenseminaren der Schweiz. Berichte der Schweizerischen Subkommission, 1912.

1828. Schiffner, F., and Müller, F. Die Ausbildung der Lehramtskandidaten in der darstellenden Geometrie. Referat und Correferat im Verein "Die Realschule" in Wien, 30. IV., 1910; Österr. Mittelschule, 25 (1911).

1829. Schnell, H. See Secondary schools.

1830. Schotten, H. Diskussion über die Dresdener Vorschläge der Unterrichtskommission der Gesellschaft Deutsche Naturforscher und Ärzte betreffend die Ausbildung der Lehramtskandidaten. Zschr. math. natw. Unt., 39 (1908): 531.

1831. Schröder, O. Die Ordnung des Studiums für das höhere Lehramt in Deutschland und die gesetzlichen Prüfungsbestimmungen in den einzelnen Bundesstaaten. Leipzig, 1906.

1832. Schulze, P. Die wissenschaftliche Ausbildung der Lehramtskandidaten der Mathematik und Naturwissenschaften nach den Vorschlägen der Unterrichtskommission. Blätter höh. Schulw., 25 (1908).

1833. Schwarzschild, K. Über die astronomische Ausbildung der Lehramtscandidaten. Vortrag auf der Göttinger Besprechung. Jahresber. D. M. V., 17 (1907). 
1834. Schwering, K. Handbuch der Elementarmathematik für Lehrer. Leipzig, 1907.

1835. Smith, D. E. The preparation of the teacher of mathematics in secondary schools. School Science and Mathematics, 7 (1907): 247.

1836. Stamper, A. W. The training of teachers of mathematics in the secondary schools of the United States. School Science and Mathematics, 10 (1910).

1837. Studien der zukünftigen Lehrer der Mathematik auf technischen Hochschulen. Zschr. math. natw. Unt., 34 (1903).

1838. Suppantschitsch, R. Die Heranbildung der Lehrer für Mathematik und der Ingenieure in Frankreich. Zeitschrift für das Realschulwesen, 37 (1911).

1839. Tanf, I. Die gegenwärtige Lage und die Reformfrage des mathematischen Unterrichts in den ungarischen Seminaren. Zschr. math. natw. Unt., 40 (1909): 74.

1840. Thomson, G. H. The training of secondary school teachers in England. Math. natw. Blätter, 4 (1907).

1841. Training of teachers of elementary and secondary mathematics. International Commission on the Teaching of Mathematics. American report. Committee No. V, E. H. Taylor, chairman. Washington, D. C., 1911. (U. S. Bureau of Education. Bulletin, 1911, No. 12.)

1842. Treutlein, P. See Elementary Schools.

1843. Umlauf, $\mathbf{K}$. See Elementary Schools.

1844. Upton, C. B. The training of teachers of mathematics in professional schools of collegiate grade. Educational Review, 41 (1911): 382.

1845. Weber, H. Wirkung der neuen preussischen Prüfungsordnung für Lehramtskandidaten auf den Universitätsunterricht. Jahresber. D. M. V., 8 (1900).

1846. Wieleitner, H. See Secondary Schools, and Correlation.

1847. Wirz, J. See Secondary Schools.

1848. Die wissenschaftliche Ausbildung der Lehramtskandidaten. Zschr. phys. chem. Unt., 21 (1908).

1849. Wissenschaftliche Vorbereitungskurse für Mittelschullehrer (dazu Klein's Rede im Herrenhaus). Lietzmann's Rubrik in Zschr. math. natw. Unt., 41 (1910). 



\section{INDEX.}

\section{A.}

Ackermann, C., 896.

Ícs, L., 1341.

Adamicka, M., 656.

Adhémar, R., 833.

Adler, A., 1, 987, 1342.

Afnassjewa-Ehrenfest, T., 988.

Ahrendt, H., 1548.

Alasia, C., 414.

Albrecht, J., 1549.

Albrich, K., jr., 415, 416, 1522, 1550.

Albricht, K., 1522.

ALGEBRA, 896-986.

Allen, F., 417.

Amaddii, I., 756.

American Mathematical Society, 418.

Amieux, Mlle., 419.

Andrade, J., 2, 268, 274, 989-990.

André, D., 1400.

Antal, M., 1523.

Apel, O., 3 .

Appell, P., 4, 381, 991, 1551-1554.

APPLICATIONS OF Mathematics, 1548-1738.

Arany, D., 657.

Arbes, J., 1482-1484.

Archenhold, F. S., 5.

ARITHMETIC, 755-895.

Arldt, 1746.

Arndt, 1043.

Ascoli, M., 1555.

Ashford, C. E., 695.

Association of Public School Science Masters, 1664.

Association Suisse de Professeurs de Mathématiques, 6-7.

Atmanspacher, 0., 757.

Aubert, J., 1485.

Ayné, P., 1401.

\section{B.}

Babb., M. J., 422, 758.

Badertscher, 423.

Bailey, M. A., 760 .

Baker, A. L., 9, 897, 993-998.

Balás, G., 10 .

Ball, W. W. R., 719.

Ballard, P. B., 382, 898.

Balser, L., 999.

Bandini, S., 761.

Bartram, H., 1557.

Bass, W., 424

Baud, 425 .

Baudeuf, H., 1000.

Bauernberger, A., 1486.

Baumgartner, A., 1242.

Beard, W. F., 11.
Beardsley, R. S., 1001.

Beck, K., 12.

Becker, V., 13.

Beischer, K., 1254.

Beke, M., 15-18, 383, 426, 762, 1255, 1748 .

Beman, W. W., 1002, 1402.

Benesch, R., 1256.

Berak, J., 1719.

Bérard, R., 1403.

Berdellé, C., 19, 763.

Bergmann, F., 427, 1560 .

Bernstein, J., 429.

Bertola, G., 764.

Bettazzi, R., 432, 1404, 1562.

Bettini, B., 22.

Betz, W., 1003-1005, 1405.

Beuriger, J., 1006.

Beyel, Chr., 1343-1344.

Bikle, C. E., 9, 899.

Bioche, Ch., 383, 433.

Bishop, F. L., 1564.

Blakslee, T. M., 24, 1406.

Bleucke, Fr., 1345.

Blum, R., 1007.

Blutel, E., 434, 721, 1258-1259.

Bobynin, V., 25-2i, 435, 722.

Bodenstedt, H., 1346-1347.

Bochnr, M., 730.

Bochow, K., 1260.

Bock, A., 409, 1750.

Bode, P.. 436.

Bo:hm, E. 28

Bognár, P. C., 1348.

Bogner, 1349.

Bogyó, S., 675 .

Bohle, G., 437.

Böhme, B., 1751.

Bond, W. E., 1008.

Bonnesen, T., 29, 438.

Boon, F. C., 1408.

Borchardt, P., 765

Borel, E., 268, 274, 439, 724, 1009.

Borgmeyer, J., 1010.

Böttcher, L., 440-441.

Böttger, W., 442.

Bourlet, C., 444, 1011-1013.

Boz6ky, E., 901, 1014.

Brand, E., 1409.

Brandenberger, K., 445.

Branfoid, B., 766.

Braunschweig, R., 1739.

Breckenridge, W. E., 30, 1566.

Bremiker, H., 902.

British Association Committee on the Teaching of Mathematics, 31.

Brocke, E., 32-33, 903, 1015. 
Brookman, T., 446.

Broomell, B. L., 34, 1740.

Brown, E. E., 447.

Brown, E. W., 1567.

Biown, F. B., 448.

Brown, J. C., 768 .

Browne, C. E., 769 .

Bruce, G. A., 9.

Brucher, K., 770.

Bruës, M., 904.

Brun, F., 385.

Bruns, P., 771.

Bryan, G. H., 1262, 1752.

Büchcl, W., 1410.

Buchrucker, B., 35, 1263, 1568.

Buffa, P., 1016.

Buhl, A., 725.

Bühler, 772

Buhlmann, B., 36 .

Burger, Ch., 449.

Burstall, S. A., 37.

Busse, M., 1569.

Bütler, K., 1264.

Buttner, A., 773.

Buyse, O.. 38 .

\section{C.}

Cailler, C., 774.

Cajori, F., 905, 1411.

Calculus, 1254-1340.

Campbell, J., 39.

Capelli, A., 906.

Cardinaal, J., 40-41.

Carson, G. St. L., 1017.

Carver, W. B., 451.

Castelli, G., 1570.

Cauer, P., 1753.

Central Association, 907.

Chabot, C., 1754.

Chase, H. J., 1018-1019.

Chatelet, A., 1020, 1755.

Chatelier, H., 660.

Chénard, H., 1021.

Chevalier, 1022.

Chiari, A., 775.

Child, J. M., 1024.

Ciamberlini, C., 42, 776, 1571.

Cikot, C. A., 1026.

Clairaut, A. C., 1027.

Clark, C. A., 1572.

Clarke, J. B., 453.

Coar, H. L., 43, 1756.

Cobb, H. E., 44-45, 1573.

Colleges and universities, 719-754.

Collins, J. V., 46-48, 777, 908, 1574.

Combet, E., 454, 1222.

Combebiac, C., 1028.

Commission Internationale de l'Enseignement des

Mathématiques, 49.

Committee on the Unification of Mathematies, 50 .

Comstock, C. E., 51, 455, 1575.

Condit, I. S., 52.

Congresso della "Mathesis," Società Italiana di Matematica, 54.

Congresso die Professori di Matematica, 55.

Congresso Internationale del Matematici, 56.

Conti, A., 72, 456, 1757-1758.
Coolidge, J. L., 58.

Cornet, L., 1268.

Cornish, W. A., 909.

CORRElation aNd applicatior's of mathematics. 1548-1738.

Corte, D., 457.

Cosamassima, M., 778.

Courcot, A., 1412.

Cramer, A., 58.

Cramer, F., 458.

Cramer, H., 409, 459.

Crawford, L., 387.

Crelier, L., 661, 1350.

Csada, I., 59, 779-780.

Csall6közi, J., 460.

Cubberly, E. P., 145.

Curtis, A. M., 910.

Czak6, A., 1577.

Czuber, E., 61, 662, 1269, 1762.

D.

Dahlgren, H., 389 .

Dalwigk, F. von, 1578.

Danielewicz, B., 911.

Daninger, J., 62, 461, 1579.

Dankwortt, A., 63.

Dannemann, F., 1764.

Darboux, G., 1581.

Darnell, A., 1413.

Darnet, L., 781.

Dausat, 64.

Davis, E. W., 964.

Dean, P., 913.

Deckars, R., 782.

Délkány, M., 1765.

Delitala, G., 1223.

Deny, L., 1414.

Dewey, J., 1030.

Dickson, L. E., 1415.

Diekmann, J., 914, 1031.

Dietrich, M., 1032.

Dietsch, C., 1033.

Dietz, C., 462.

Dintzl, E., 463-464, 1224, 1524.

Dittrich, E., 68.

Ditzel, H., 465.

Dobbs, W. J., 1488.

Doerge, O., 1034.

Dolězal, E., 1416.

Dolinski, M., 663.

Donecker, F. C., 916.

Dooley, W. H., 785.

Doolittle, F. W., 1421.

Dougall, J., 1418.

Dra WiNG, Geometrical, 1341-1399.

Dressler, H., 390, 917, 1035, 1525, 1582, 1766.

Ducrue, J., 1036, 1352.

Dumas, H., 466.

Dunker, K., 1583-1584.

Durand, A., 1037.

Durrell, C. V., 786, 1244.

Durrell, F., 918, 1038-1040.

Düsing, K., 69, 1271.

E.

Ebner, F., 467, 1272-1273.

Eckhardt, E., 919 . 
Eggar, W. D., 1585-1586.

Ehrenfest, T., 988.

Ehrig, G., 664

Eickhoff, R., 1767.

ELEMENTARY SCHOOLS, 381-413

Emch, A., 1587.

Engelhardt, P., 1420.

Epps, F., 787.

Epstein, S., 1421.

Erikson, G., 665.

Enriques, F., 72, 728.

Erdmann, 1043.

Erdmann, R., 468.

Erdös, L., 73.

Escherich, G., 729, 1768.

Eyans, G. W., 473, 542, 920, 1044-1045, 1422 .

\section{F.}

Fabinger, F., 1046.

Fack, M., 76.

Farag6, A., 77-78, 474, 1047.

Färber, C., 788.

Faulland, J., 922, 1590.

Fazzari, G., 789.

Fehr, H., 80-85, 475-476, 1048, 1526-1527, 1769.

Fick, A., 1591.

Fick, E., 790.

Ficquet, M., 477, 1049 .

Field, F., 87.

Filon, L. N. G., 1592.

Finsterbusch, J., 1050.

Fischer, K. T., 1593, 1771.

Fiser, P. R., 1245.

Fiske, T. S., 74.

Flatt, R., 478.

Flegel, W., 1353.

Fochtmann, 1051

Fontené, G., 89.

Förster, W., 1594.

Forsyth, A. R., 1595.

Forth, C. J., 90.

Francis, W. A., 1052, 1596.

Frank, H., 1274.

Franz, J., 1257.

French, J. S., 91, 480-481.

Frenzel, C., 1276.

Freud, $\mathrm{Ph} ., 482$.

Fricke, F., 1225.

Fricke, K., 1597, 1772.

Fricke, R., 92.

Friedel, V. H., 1773.

Friemel, R., 1598 .

Fries, W., 1774.

Frischauf, J., 791, 1053.

Frixon, H., 666.

Fröhlich, K., 146, 792 .

Füger, J., 483.

Function concept, INTRODUCTION of, 1522-1547.

Furtwängler, P., 667 .

G., C., 1054 .

G.

Gaffiney, M. A., 1055.

Gajdeczka, J., 1776.

Galdéano, Z. G., 94-99, 1599.

Gale, A. S., 391, 484.

Gallander, O., 1423.

Gallucci, G., 485-486.
Garbieri, G., 274.

Gaubatz, M., 1354-1355.

Gauss, A., 793.

Gebhardt, M., 1741-1742.

Geck, E., 409, 487.

Gehrig, H., 392.

Geiger, K., 794.

Geissler, K., 100-102, 1528-1529, 1600.

Gendame de Bévotte, 1778.

GeNeral topics, $1-380$.

Genese, R. W., 923.

GEOMETRY, 987-1221.

GEOMETRY, ANALYTIC, 1242-1253.

GEOMETRY, DESCRIPTIVE, 1341-1399.

Gérard, L., 103.

Gercken, W., 489, 1356 .

Geissler, K., 1528-1529.

Gerhardt, R., 1601-1602.

Gerlach, A., 795, 1056.

Gheury, M. E. J., 105.

Gibson, G. A., 106.

Giebel, K., 492, 1603.

Gille, A., 1057 .

Girndt, M., 669.

Glauer, R., 1226, 1489.

Glauner, Th., 1604.

Glenn, O. E., 1424.

Göbelbecker, L. F., 796.

Godfrey, C., 107-108, 171, 924-925, 1058-1059.

Goering, W., 1530.

Goldziher, C., 109-116, 146, 670, 797- $798,926-927$, 1425-1430, 1605-1606, 1779 .

Goller, 493, 1780 .

Goodrich, M. F., 928.

Göransson, E., 117-119, 494-495, 504

Götting, E., 143, 496-497.

Gottschalk, A., 328.

Gouilly, A., 1607 .

Goy, P. de, 1431.

Graf, J. H., 731 .

Graham, E., 498.

Graphs, Value and Teaching of, 1400-1481.

Grave, W., 121.

Greenhill, Sir G., 1608.

Greenstreet, W. J., 740.

Greenwood, G. W., 929, 1060-1065.

Grévy, A., 122

Grimm, O., 799.

Grosse, W., 1432, 1609-1610.

Grossmann, M., 123, 671, 1066.

Grothmann, H., 1357 .

Grühl, P., 930.

Grünbaum, H., 672 .

Grunewald, W., 1358.

Guadagno, P., 124.

Gubler, S. E., 500.

Guerritore, G., 1611.

Guldberg, A., 125.

Günther, S., 126.

Gutzmer, A., 127-130, 733, 1612, 1781, 1794.

Guyon, E., 800 .

Gwatkin, E. R., 501.

H.

Haacke, C., 801.

Haacke, F., 1067, 1278-1279.

Haage, K., 1359. 
Haas, M., 1068.

Habán, M., 1280.

Häbler, Th., 1227.

Hack, 1531.

Hadamard, J., 131, 1613.

Haentzschel, E., 1069.

Haese, 673.

Haeseler, O., 503.

Haffner, G., 1070.

Hagström, K. L., 674.

Haidl, H., 931.

Hall, E. H., 1614.

Hallgren, E., 504.

Halsted, G. B., 1071, 1615.

Hamilton, J. G., 1072.

Hammer, E., 1490.

Harper, G. A., 1616.

Hart, C. A., 1073.

Hart, H. F., 802, 1074-1075.

Hartmann, B., 803.

Haskins, C. N., 691.

Hasse, W., 505.

Hatzidakis, N. J., 734.

Hauck, G., 1617.

Havas, M., 676.

Hawkes, H. E., 132, 735, 1076.

Hawkins, C., 133.

Hayashi, T., 1433.

Heaviside, O., 134.

Hecht, C., 506.

Hecht, W., 1618.

Heck, R. C. H., 1434.

Heckscher, I., 135.

Hedgepeth, V. W. B., 804.

Hedrick, E. R., 805, 932-933, 1077.

Heffter, L., 1281.

Heininger, 806 .

Heinrich, M., 1078-1079.

Hellweger, M., 1080.

Hensing, H., 409.

Hermes, J., 1619.

Herting, G., 1282.

Heumann, C., 677.

Hiemesch, R. H., 807.

Hill, M. J. M., 1081.

Hinckley, F. C., 1491.

Hioux, V., 1082.

Hobson, E. W., 136-137.

Hočevar, F., 808, 1283.

Höckner, G., 1622.

Hoefinghoff, E., 138.

Höfler, A., 139-141, 507-j10, 1624, 1783.

Hoffmann, B., 1623.

Hogben, G., 1083.

Hohlstein, P., 1361.

Holmes, H. I., 142.

Holzmüller, G., 143, 1284-1285, 1784.

Hoppe, E., 1286.

Horn, E., 511.

Hornig, 512.

Hossfeld, C., 513.

Hovestadt, H., 158, 815.

Hubbes, H., 1287.

Huber, 1625.

Huguenard, E., 809.

Hume, A., 514.

Huntington, E. V., 678 .

Hurst, G. H. G., 1626.

\section{I.}

International Commission on the Teaching of Mathematics, Committees nos. I and II, 400; Committees nos. III and IV, 542; Committee no. V, 1841; Committee no. VI, 691; Committee no. VII, 74; Committee no. VIII, 145; Committee no. IX, 692; Committee no. X, 750; Committee no. XI, 690; Committee no. XII, 729.

\section{J.}

Jackman, W. S., 147.

Jackson, C. S., 148, 810, 1492, 1627.

Jackson, D. C., 1628.

Jackson, L. L., 811.

Jackson, W. H., 1084.

Jacob, J., 1768.

Jacobs, A., 1629.

Jacquemir, A., 1630.

Jahn, O., 812.

Jahnke, E., 679.

Jamieson, W. R., 1631.

Janisch, W., 1085-1086, 1228.

Janzen, O., 934.

Johanneson, P., 1632.

Jones, F. T., 150, 935.

Josephson, O., 596, 1532, 1786.

Jourdain, P. E. B., 151.

Joxe, A., 152.

Juckel, Gy., 153, 813-814.

Jung, P., 1633.

Junge, G., 154-155, 1493, 1634.

\section{K.}

Kabisch, O., 1787.

Kántor, N., 1288.

Kármán, T., 156, 680.

Karpinski, L., 157.

Keferstein, H., 936.

Kelemen, I., 1088.

Kemlein, G., 1089, 1247.

Kendall, C. N., 400.

Kerschensteiner, G., 409.

Kewitsch, G., 1289.

Keyser, C. J., 158.

Killing, W., 159, 815.

Kinkaid, E. M., 1635.

Kirchberger, R., 816, 1362.

Kiseljak, M., 938.

Kissel, F., 1636.

Kleefstra, J., 1637.

Klein, F., 160, 517-520, 736-737, 1638-1645, 1789-1794.

Kleinpeter, H., 161, 521-522, 939, 1090, 1795.

Klug, J., 162.

Klug, R., 1091.

Knabe, K., 163.

Knabl, E., 1646.

Kobalt, E., 682 .

Koch, E. H., 164.

Koch, H. von, 683.

Koch, W., 1092.

Koeppner, K., 1248 .

Kolhtsch, A., 817.

Kollros, L., 165.

Kondratiev, V., 523.

Konrath, Th., 524, 1796.

Kopp, L., 1093.

Kosmik, K., 1094. 
Kraus, K., 397, 818.

Krause, M., 525, 1797.

Krebs, W., 1798.

Kretzschmar, J. F., 526.

Kreuschmer, R., 1229.

Krimphoff, W., 1647.

Krollik, H., 527 .

Kronke, F., 1363.

Krug, 940.

Krüse, K., 941.

Kubassa, C., 1290.

Kühn, F., 819, 1095.

Kuhn, I., 1435.

Kühnemann, F., 166.

Kullrich, E., 1096, 1364, 1494.

Kürschák, J., 1799.

\section{L.}

Lacombe, M., 684.

Laisant, C. A., 167-170, 685, 1097, 1291.

Langley, E. M., 171.

Langlois, C. V., 1800.

Lanner, A., 172, 820, 1230.

Laparevicz, A., 1648.

Lasher, W. R., 1098.

Lazzeri, G., 686.

Lechnitczky, G., 173.

Lefèvre, A., 1099.

Lehmer, D. N., 174.

Lehrfeldt, R. A., 1436.

Lémeray, E. M., 1292.

Lemoine, J., 1649.

Lengauer, J., 176.

Lennes, N. J., 942-943, 1100-1101, 1437.

Lesser, O., 1438, 1533.

Lessing, Th., 177.

Levi, B., 178.

Lexis, W., 179.

Leygues, 180.

Liard, L., 1102, 1650-1651.

Lietzmann, W., 181-186, 398, 529-531, 821-822, 11031106, 1652-1655, 1804.

Liewald, K., 1107.

Lilienthal, R. von, 738,1656 .

Liman, O., 532.

Lindemann, F., 187.

Lissák, J., 944, 1495.

Lodge, O., 188.

Löffler, E., 189, 1108.

LOGARITHMS AND THE SLIDE RULE, 1482-1521.

L6ky, B., 534.

London. Board of Education, 1108.

Long, E., 1657.

Loomis, H. B., 945.

Loos, J., 190, 1805.

Lopiě, P. J., 824.

Lörcher, A., 1110.

Lorenz, H., 1658.

Lorenz, K., 825.

Lorey, W., 191, 535-536, 739, 826, 1231, 1293-1296, 1806.

Loria, G., 192-194, 268, 274, 537-538.

Low, D. A., 688.

Luckhaub, G., 1297.

Ludwig, F., 1659.

Lytle, E. B., 1111.

\section{M.}

Macaulay, F. S., 740.

McCormack, T. J., 196.

McDill, R. N., 946.

Mach, A., 1660.

Mach, E., 195.

McIntosh, J., 1661.

Mack, K., 1112.

McKelden, A. M., 947.

McKinney, T. K., 948.

McLeed, C., 1496.

McMurry, C. A., 826.

McMurry, F. M., 403.

McPherron, J. M., 1113.

Maestro, T., 689.

Mahistre, G., 197.

Makchéev, M., 1807.

Málly, F., 539.

Maltson, R., 1534.

Mannheim, A., 1114.

Mannowry, G., 198.

Marjanek, A., 949.

Marotte, F., 199, 268, 540, 1115, 1439, 1662-1663.

Martin, P., 1116-1117.

Massau, J., 1365.

Mathematical Association, 200-201, 1664.

Matter, K., 693

Maurer, A., 1665.

Mayer, 204.

Mayer, T. E., 1497.

Mehmke, R., 828, 1440.

Meissner, O., 1366.

Meldau, H., 709

Mende, J., 829.

Mendelssohn, V., 1535.

Méray, C., 205, 1118-1120.

Mercer, J. W., 695.

Messineo-Cantarella, S., 830.

Mettler, H., 1441.

Metzler, W. H., 206-208.

Mevius, W., 209.

Meyer, A., 119.

Meyer, E., 543, 1498.

Meyer, Fr., 544.

Meyer, U., 211.

Mikola, S., 18, 212-214, 426, 831, 1667.

Mikuta, A., 696.

Milankowitsch, M., 1442.

Milarch, E., 1298-1299, 1499.

Miller, G. A., 215-218, 1121, 1743, 1809.

Millis, J. F., 950, 1668-1669.

Milne, R. M., 1443.

Milthaler, J., 546.

Minchin, G. M., 1122.

Mineur, P., 1500.

Misar, W., 219.

Missouri Society of Teachers of Mathematics and

Science, 951.

Mittag, M., 1444.

Molliverg, 548.

Möller. M., 832.

Molnár, E., 1300.

Montessus, R. de, 833 .

Moore, E. H., 1445.

Moore, M. E., 1810.

Mootschek, W. de, 220. 
Morawetz, J., 834, 1123.

Morf, L., 697.

Moritz, R. E., 1232, 1501.

Moroff, A., 1124, 1233.

Morsch, H., 1811.

Most, S., 549.

Muir, D., 221.

Muk, H., 1125.

Müller, C. H., 1502.

Müller, E., 1367-1368.

Müller, F., 1670, 1812.

Müller, H., 222, 550-551, 1249, 1828.

Müller, R., 1126.

Munchoff, E. R. von, 223.

Muthesius, K., 1814.

Myers, G. W., 552-555, 953, 1503-1504, 1671.

Myller, A., 224.

\section{N.}

Nagele, L., 1301.

Nager, J., 1127.

Nannei, E., 556.

Nath, M., 225, 557-561, 835, 1128, 1815-1816.

Natucci, A., 226, 274, 562, 836-837.

Neuberg, J., 563.

Neuhoff, H., 227.

Neurath, O., 1672.

Newbold, W., 566.

Newcomb, S., 838-839.

Newhall, C. W., 567-569,954.

Nielsen, C., 1130.

Niewenglovski, B., 228.

Nitsche, O., 840, 1673.

Noaillon, P., 1446.

Noble, C. A., 570.

Nodnagel, 571.

Nonne, Th., 1447.

Noodt, G., 572-573, 955, 1131, 1448.

Nordlund, K. P., 956.

Norrenberg, J., 1132.

O.

Oberbach, J., 1449.

Oberle, K., 229.

Oblath, R., 576 .

Ocagne, M. d', 1450-1452, 1505.

Orbán, G., 958, 1453.

Osborn, C. S., 959.

Osgood, W. F., 272, 1303-1304.

Ott, C., 698.

Otta, M., 1305 .

Otti, 1506.

\section{P.}

Packard, J. C., 232, 578, 1675.

Paetz, W., 841.

Pages, A., 1454.

Pahl, F., 1676.

Palatini, F., 233.

Palmer, G. W., 842.

Palotay, A., 579.

Pannwitz, R., 1133.

Pařezek, A., 843.

Patterson, C. A., 1134-1135.

Paulsen, F., 234.

Péch, A., 1455.

Peck, P. N., 1136.

Pedoth, A., 844.

Penndorf, B., 699.

\author{
Penseler, G., 235. \\ Perrin, E., 1137. \\ Perry, J., 236-240, 1677-1678. \\ Peters, F. N., 1679 \\ Petit, G., 1138. \\ Petrini, H., 241. \\ Pfaff, H., 1139. \\ Pfitzner, P., 242. \\ Phillippowitsch, F., 220. \\ Pierpont, J., 244.
}

Pietzker, F., 245-250, 1369, 1680.

Pillet, F. J., 1370.

Pincherle, S., 274, 742.

Plttarelli, G., 1818.

Plasche, R., 252.

Pochin, E. A. N., 253.

Poincaré, H., 254-255.

Polakoff, S., 582.

Poprugenko, M., 701.

Poske, F., 256.

Possé, C., 743.

Postelmann, E., 1682.

Poussin, R., 1456.

Pradel, C., 583.

Pratt, A., 1457.

Presler, O., 1683-1684.

Pressland, A. J., 1685.

Prieur, 1458.

Pringsheim, A., 257.

Privorszky, A., 258.

Puller, 960.

Putney, E. N., 1140.

Pyle, L., 1686.

Queisser, F., 260.

Q.

Quint, N., 1507.

Quossek, K., 261-263, 587, 1371-1372, 1536.

Rabes, O., 961.

R.

Rados, I., 146, 264.

Rados, G., 703.

Rais, 806.

Ramaswami, A., 1306.

Ramsay, W. W., 1491.

Rátz, L., 1308.

Reich, K., 704.

Reiff, R., 590 .

Reinhardt, C., 1688.

Reinhardt, R., 1373, 1824 .

Reisky, S., 1141.

Reitmann, E., 271.

Renbach, W., 1374.

Report of the American commissioners, 272.

Ricci, G., 274.

Richard, J., 1143.

Richert, P., 1144, 1459.

Richter, A., 594-595, 705-706, 1375, 1744.

Richter, H., 1376.

Richter, M., 1377.

Richter, O., 1145, 1378, 1509, 1689.

Riechemeier, W., 1146.

Riecke, E., 1644-1645.

Riehl, A., 1690.

Riesz, F., 273.

Rietz, H. L., 962-963.

Rigge, W. F., 1147.

Ripert, L., 1148-1149. 
Roe, E. D., 1150-1151.

Rohrauer, G., 1537.

Rohrberg, A., 275.

Rollet, P., 707.

Rönstrom, A., 596.

Rorer, J. T., 276, 846, 1152.

Rose, J., 277, 1250.

Rottsieper, W., 1460.

Roubalt, 1691.

Roubaudi, C., 1379-1380.

Rouse, E. L., 964.

Rousseau, H., 1154.

Row, T. S., 1155.

Rozé, P., 1510.

Ruault, 1692.

Rudel, E., 1693.

Ruediger, W. E., 1825.

Rühlmann, K., 1381.

Rühlmann, R., 278.

Rulf, W., 708.

Ruska, J., 1826.

Russell, B., 1156.

\section{\$.}

S., B., 402.

St.-Germain, A de, 744

Sainte Laguë, A., 279, 847-848, 1157-1158, 1461.

Salomon, A., 1159.

Salow, A., 1462.

Sandars, A., 1160.

Saposnikov, A., 965.

Saunders, S. A., 849.

Saxelby, F. M., 280.

Schacht, J., 1161-1163.

Schaewen, P. von, 1164-1165.

Schäffer, A., 281.

Scheibner, O., 282.

Scherrer, F. R., 1827.

Schiede, J., 1310.

Schiffiner, F., 1828.

Schilling, C., 709.

Schilling, F., 1383.

Schilling, G., 1695.

Schimmack, R., 283-285, 520, 1311.

Schlags, W., 1463-1469.

Schlesinger, J., 286, 1167.

Schlesser, E., 598.

Schlosser, A., 599, 1235.

Schmall, C. N., 1312.

Schmehl, C., 1167, 1236, 1384, 1538.

Schmid, B., 287.

Schmidt, A., 1511.

Schmidt, J., 1213-1314.

Schmidt, O., 1696-1697.

Schmidt, W., 1539.

Schmitz-Mancy, M., 288.

Schnee, W., 1540.

Schneider, 850 .

Schneider, F., 289.

Schnell, H., 600 .

Schöngut, L., 1315.

Schotten, H., 290-295, 1385, 1465, 1698-1700, 1830.

Schoute, P. H., 1251.

Schrautzer, K., 1315.

Schreber, K., 1541.

Schröder, J., 601-602, 1237, 1466.

Schröder, O., 1831.
Schröter, K., 1317.

Schubert, H., 1512.

Schuberth, A., 1386.

Schülke, A., 296-300, 851, 967-968, 1168, 1273, 1318 $1320,1513-1514,1701-1703$.

Schulte, T. H., 1169.

Schultz, E., 1170, 1321, 1542.

Schultze, A., 1171.

Schulze, E., 302.

Schulze, E. W., 1172-1173.

Schulze, P., 1832.

Schumacher, R., 1515.

Schumann, E., 1322.

Schuster, M., 303, 1174.

Schütte, Fr., 1387.

Schwacha, P. B., 852.

Schwarte, A., 1704.

Schwarz, P., 853.

Schwarzschild, K., 1833.

Schwatt, I. J., 304-305, 604, 1175.

Schwering, K., 306, 605, 1834.

Schweter, R., 854.

Scorza, G., 710 .

Scott, C. F., 711 .

SECONDARY SCHOOLS, 414-655.

Sedgwick, Mrs. H., 745.

Seidler, H., 969, 1252.

Sellenthin, R., 1388-1389.

Servaty, J., 1745.

Servit, F., 1176.

Severi, F., 72 .

Shaw, C. A., 855.

Sherk, W. H., 117 .

Shobinger, J. J., 606-607.

Short, R. L., 371, 608-609, 970.

Shutts, G. C., 1178.

Siddons, A. W., 171.

Sieber, A., 1467.

Sievert, H., 1033, 1179.

Simon, M., 310-313, 1180-1181

Simon, O., 610.

Simons, L. G., 9 .

Simony, O., 713.

Sintsof, D., 611.

Sisson, E. P., 1182

Skinner, E. B., 612, 1183.

Slater, C. H., 1705.

Slaught, H. E., 316-318, 971-972, 1184-1185.

SLide Rule. See Logarithms.

Slocum, S. E., 319-320.

Smith, A. W., 1468.

Smith, D. E., 268, 272, 274, 321-326, 403, 613, 856-858, 973-974, 1186, 1706, 1835.

Smith, E. R., 9, 975, 1187-1189.

Snow, L. F., 746.

Snur, H., 328 .

Soecknick, K., 329, 1190.

Somervell, E. L., 330 .

Somigliana, C., 747.

Sommer, J., 1238.

Sommerville, F. H., 976.

Sos, E., 1469-1470.

Souls, 331.

Sourek, A.-V., 332 .

Spencer, H. J., 404.

Spiess, J., 1516.

Stäckel, P., 713, 1707-1708. 
Stamper, A. W., 1191, 1836.

Starch, D., 859.

Stark, W. E., 542, 614.

Steckelberg, H., 1323.

Steinbart, O., 333.

Stephens, I., 405.

Sterneck, R. von, 748 .

Steuer, W., 860.

Stiessen, M., 1192.

Stitt, E. W., 861.

Stöcklin, J., 406.

Stone, C. W., 862.

Stone, J. C., 94 ?.

Story, L., 615.

Story, W. E., 334.

Strayer, G. D., 400, 407.

Streng, K., 863.

Stromeyer, C., 335-336.

Strong, C., 337.

Stscherbina, K., 616.

Study, E., 355.

Sturm, 1709.

Stuyvaert, M., 1471.

Suppantschitsch, R., 339, 617, 864, 1193, 1517, 1838.

Sutton, C. W., 1194.

Suzzallo, H., 865.

Sykes, M., 340, 1195.

Szab6, D., 1239.

Szabo, G., 146, 1710.

Szab6, P., 618, 1196-1197.

Szántó, H., 1472.

Szenes, A., 619, 866.

Szijárto, M., 1473.

Szücs, A., 342, 1198.

Szücs, E., 1240.

Tanfi, I., 343, 408, 1543, 1839.

Tanikiewits, V., 1474.

Tannery, J., 344-345, 1199.

Taylor, A. R., 978.

Taylor, C. K., 1200.

Taylor, J. M., 979.

Teachers, Preparation of, 1746-1815.

TECHNICAL SCHOOLS, 656-718.

Terry, H. S., 980.

Tešar, L., 1324, 1711-1712.

Thaer, A., 620-622, 1325-1326.

Thiede, J., 1201, 1327, 1544.

Thiemayer, K., 1713.

Thieme, H., 1202-1203, 1328, 1714.

Thijn, A., 981.

Thomson, G. H., 1840.

Thomson, H. I., 1475.

Thorndike, E. L., 867-868.

Thurston, E. L., 1715.

Tiebe, A., 1716.

Tiedge, E., 1390.

Timerding, H. E., 1204-1205, 1329, 1717-1718.

Tinsley, S. B., 623.

Toan, B. E., 982.

Torrès, L., 1476.

Touton, F. C., 1477.

Tower, M. E., 1719 .

Townsend, E. J., 749.

Tresse, A., 983, 1518.

Treutlein, P., 348, 409, 1206-1208, 1720.
TRIGoNometry, 1222-1241.

Tripard, L., 870-871.

Tripp, M. O., 984.

Tuckey, C. O., 1330.

Twrdy, K., 1391.

Tyler, H. W., 624, 716, 692.

Uhlig, G., 349.

\section{U.}

Umani, A., 1571.

Umlauf, K., 410.

Unger, F., 1722.

Upshall, C. W., 1723.

Upton, C. B., 690, 1844.

Urban, A., 1331.

Utescher, O., 872 .

Vaes, F. J., 350 .

Vailati, G., 627-630.

Valiron, G., 873.

Vallée-Poussin, C. de la, 351.

Vareil, A., 1209.

Vatuzzi, A., 874.

Verein deutscher Ingenieure, 353, 631.

Veronese, G., 632, 1210.

Versammlung deutscher Philologen und Schulmänner in Basel, 354.

Versammlung deutscher Philologen und Schulmänner in Bonn, 355.

Visnya, A., 356, 635.

Vital, A., 1519.

Vogel, E., 1725.

Vogt, H., 636.

Vogt, K. W., 637.

Volenszky, Gy, 638.

Vollprecht, H., 876 .

Volpi, R., 1211.

Voss, A., 358.

Vuibert, H., 639.

Wägler, E., 1364.

W.

Wagner, I., 877.

Wahlgren, A., 640.

Walckling, R., 359, 1726.

Waldvogel, J., 641.

Wallentin, J. G., 878, 1727.

Wallis, 1478.

Walsemann, H., 879 .

Walsh, C. B., 642 .

Walther, F., 360, 1212-1213.

Wangerin, A., 1728.

Ward, F. L., 148, 361.

Weber, H., 362, 1845.

Webster, F., 880 .

Weill, A., 1479-1480.

Weinbeer, E. W., 1394.

Weinmeister, P., 1332.

Weise, K., 1395.

Weisner, 1520.

Weiss, F., 363 .

Weist, H., 986, 1214-1215.

Weit, 806 .

Well, G. J. van de, 1333.

Wendler, A., 364, 1241, 1334, 1545-1547.

Werkmeister, P., 1335,1481.

Wernicke, A., 365, 1730. 
Westman, J., 1731.

White, H. S., 750, 1253.

White, R. D., 1732.

White, W. F., 881-882.

Whitford, E. E., 1216.

Wieleitner, H., 366, 644, 883-885, 1733.

Wilcynski, E. J., 752.

Wilk, E., 645, 886.

Williams, R. P., 887.

Williams, S., 887.

Wilson, W. H., 753.

Wiman, A., 754.

Wimperis, H. E., 36\%.

Winch, W. H., 889-890.

Wines, L. D., 646.

Winter, J., 1521.

Wirz, J., 647.

Witting, A., 368-369, 648, 1217, 1734-1735.

Wolff, A., 1218.

Woodruff, E. C., 1736.

Woodward, R. S., 718.

Woolen, H., 370.
Wooley, J. F., 964.

Wormell, R., 1737.

Wottling, A., 649.

Wright, H. C., 371.

Wychgram, J., 650 .

Y.

Young, J. W. A., 272, 372-376, 412, 651-653, 1738.

Young, W. H., 377, 890.

Z.

Zahradniček, K., 1336-1339.

Zaremba, S., 413.

Zdelar, M., 378.

Zeissig, E., 1221.

Zenhain, 806.

Ziegler, 892-893.

Ziehen, J., 655.

Zimmermann, F., 1340.

Zoll, O., 894.

Zuckersdorfer, J., 863.

Zühlke, P., 1396-1399.

Zweger, M., 380. 
<smiles>C12C3C1C23</smiles> 


\section{BULLETIN OF THE BUREAU OF EDUCATION.}

(Continued from p. 2 of cover.)

No. 7. Undergraduato work in mathematics in colleges and universities.

No. 8. Examinations in mathematies.

No. 9. Mathematics in technological schools of collegiate grade.

No. 10. Bibliography of education for 1909-10.

No. 11. Bibliognaphy of child study for the years $1908-9$.

No. 12. Training of teachers of elementary and socondary nathematics.

No. 13. Nathematies in elementary schools,

No. 14. Provision for exceptional children in the public schools.

No. 15. The educational systen of China as recently reconstructed H. E. King

No. 16. Mathematice in Mublic and private secondary schools.

No. 17. List of publications of the United States Bureau of Education, October, 1211.

No. 18. Teacher's certificates (laws and regulations), Harlan Updegraff.

No. 19. Statistics of State universities, te, $1910-11$

\section{2.}

No. 1. Carrse of study for mul-ohool teachers. Fred Mutchler and W. J. Craig.

No. 2. Nathematics at West Point and Annapolis,

No. 3. Report of cominittee on uniform records and reports.

No. 4. Mrathematics in technical secondary schools.

No. 5. A study of xpensed of city school systems. Harlan Updegraff.

No. 6. Agricultumil education in secondary schools.

No. 7. Educational status of nursing $\mathbf{M}$. Adelaide Nutting.

Ao. 8. Peace day. Fannie F. Andrews.

No. 9. Country schools for eity boys. William Starr Hyers.

No. 10. Bibliography of education in agriculture and home economics

No. 11. Current educational topies, No. I.

Ho. 12. Dutch schools of New Netherland and colonial Nery York. W. H. Kilpatrick.

No. 13. Influences tending to improve the work of the teacher of mathematics.

No. 14. Report of tho American commissioners on the teaching of mathematics.

No. 15. Current educational topics, Nó. IL.

No. 16. The reorganized school playground. Henry S. Curtis.

No. 17. The Montessoti system of education. Anna Tolman Smith.

No. 18. Teaching Ianguage through agriculture and domestic science, M. A. Leiper

No. 19. Professional distribution of college and university graduates. B. B. Burritt

No. 20. Readjustment of a rural high school to needs of the community. H. A. Brown.

No. 21. Urban and rural common-school statistics. H. Updegraff and W. R. Hood.

No, 22. Public and private high schools.

No. 23. Special collections in libraries. W. D. Johnston and Isadoro G. Mrudge.

No. 24. Current educational topics, No. III.

No. 25. List of pubilications of the United States Bureau of Education, 1912.

No. 26. Bibliography of child study for the years $1210-1911$.

No, 27. History of public school education in Arkansas. Stephen B. Weeks.

No. 28. Cultivating school grounds in Wake County, North Carolina. Zobulon Judd. 



\section{PLEASE DO NOT REMOVE CARDS OR SLIPS FROM THIS POCKET \\ UNIVERSITY OF TORONTO LIBRARY}

Z

Smith, David Eugene

5818

M3S6

Bibliography of the teaching of mathematics, 1900-1912 


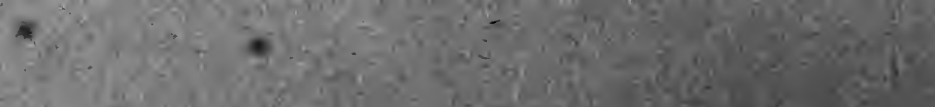

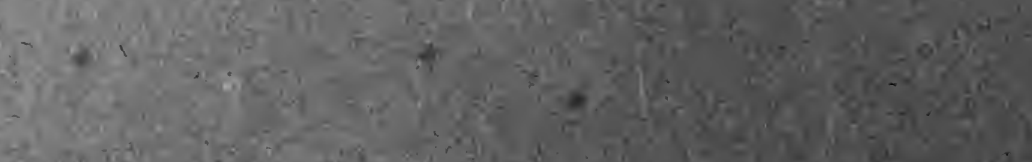
x.

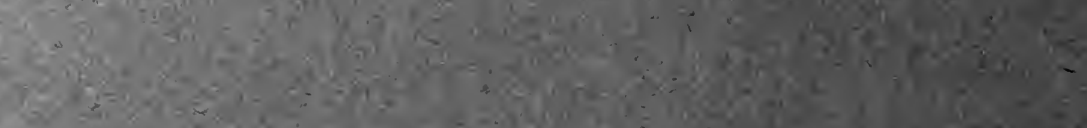

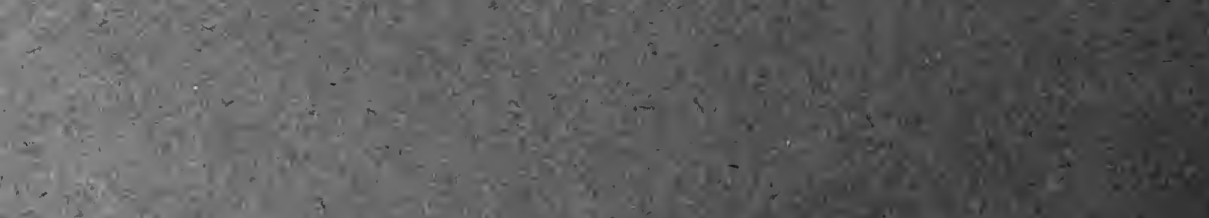

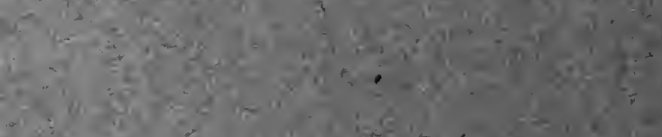

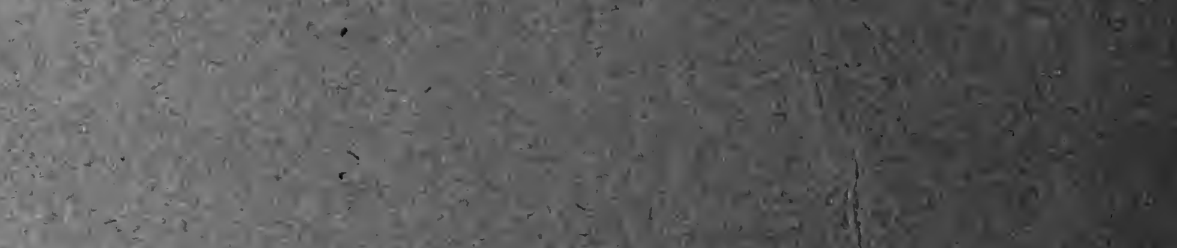
38

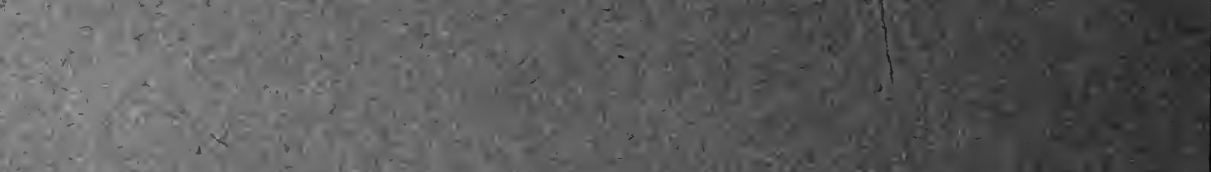

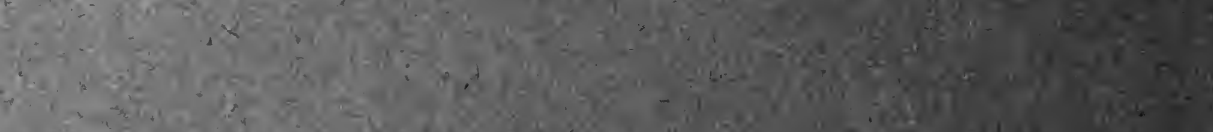

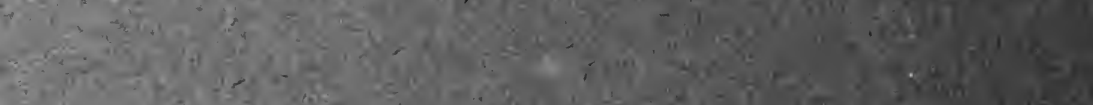

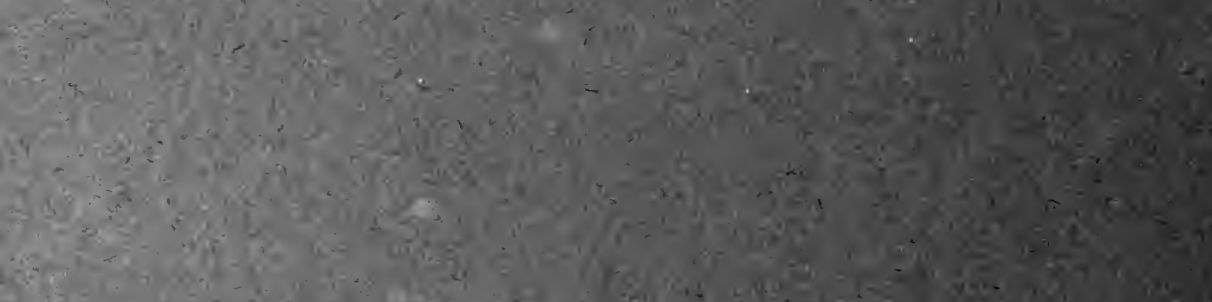

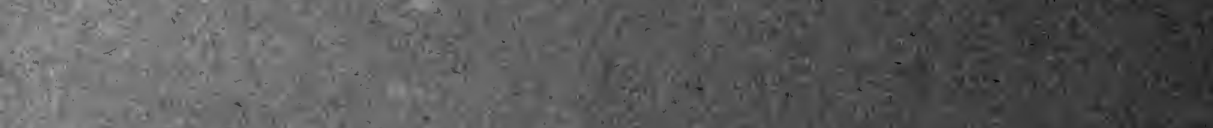

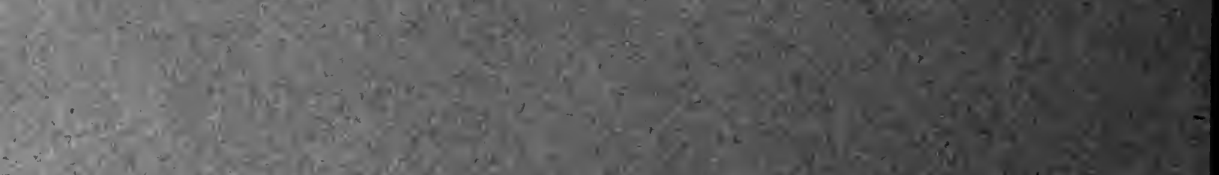

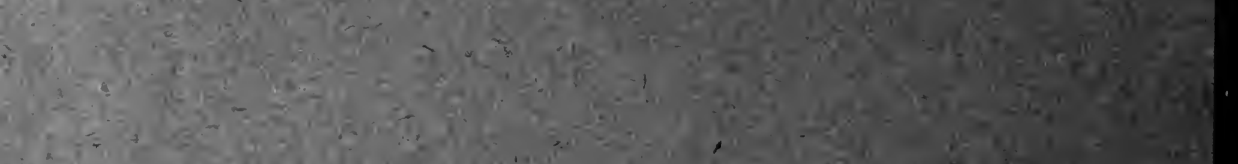

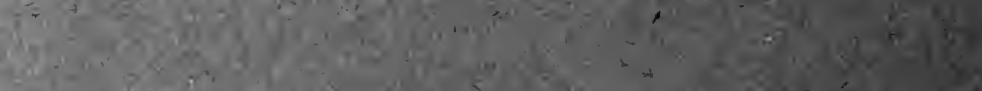
$\left.89 x^{2}+x^{2}+x^{2}\right)^{3}$

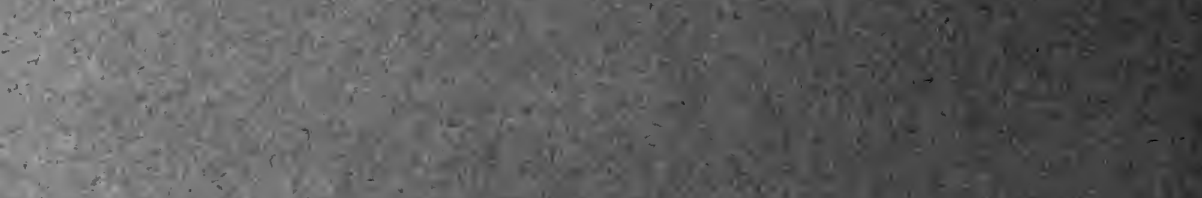

